\title{
Modelos de Riscos Híbridos com Estresse Limiar
}

Cynthia Arantes Vieira Tojeiro

DISSERTAÇÃO APRESENTADA

$\mathrm{AO}$

INSTITUTO DE MATEMÁTICA E ESTATÍSTICA

DA

UNIVERSIDADE DE SÃO PAULO

PARA

OBTENÇÃO DO TÍTULO DE DOUTOR

EM

ESTATÍSTICA

Área de Concentração: Estatística

Orientador: Prof. Dr. Francisco Louzada Neto

Co-orientador: Prof. Dr. Heleno Bolfarine

Durante a elaboração deste trabalho a autora recebeu apoio financeiro parcial da CAPES.

- São Paulo, fevereiro de 2006 


\section{Modelos de Riscos Híbridos com Estresse Limiar}

Este exemplar corresponde à redação final da tese devidamente corrigida e defendida por Cynthia Arantes Vieira Tojeiro e aprovada pela Comissão Julgadora.

São Paulo, 10 de fevereiro de 2006.

Banca examinadora:

- Prof. Dr. Francisco Louzada Neto (orientador) - Des/UFs'Lar

- Prof. Dr. Heleno Bolfarine (co-orientador) - IME/USP

- Prof. Dr. Enrico Antônio Colosimo - DE/UFMG

- Prof. Dr. Hélio dos Santos Migon - DE/UFRJ

- Prof. Dr. Josemar Rodrigues - Des/UFSCar 
Os caminhos que trilhamos são repletos de dificuldades. Cabe a nós encará-los como obstáculos ou ensinamentos.

Para Ruy, Guilherme e Gabriel 


\section{Agradecimentos}

A conclusão deste trabalho encerra mais uma etapa importante de minha vida. Esta conquista, no entanto, não teria sido atingida sem a participação de muitos, aos quais não posso deixar de agradecer.

- A Deus por mais esta realização em minha vida

- Ao professor Dr. Francisco Louzada Neto, pela dedicada orientação, pela confiança em mim depositada e experiência transmitida durante o desenvolvimento deste trabalho. Aprendi muito com sua forma de trabalhar.

- Ao professor Dr. Heleno Bolfarine, meu segundo orientador, agradeço sua confiança, paciência e suas valiosas sugestões que tornaram este trabalho possível.

- Ao professor Dr. Enrico Colosimo, com quem muito aprendi, e que mostrou-se amigo, interessado e muito contribuiu nesta conquista.

- Aos professores do IME-USP pela oportunidade de aprender e poder construir minha sólida formação acadêmica.

- Aos meus colegas de Doutorado em Estatística, em especial à Gisela, Lourdes, Michel, Adriano e Ruan, pelo companheirismo e apoio.

- As minhas amigas Gleici e Vera, pelas valiosas dicas e sugestões.

- Aos meus queridos pais, que durante toda a vida sempre me proporcionaram as melhores oportunidades de vivência e estudo. A eles e aos meus irmãos que sempre torceram e me acompanharam em todas as etapas de minha formação. Meu muito obrigado por terem me ensinado a crescer, a decidir, a lutar e a amar. 


\section{Sumário}

1 Introdução 1

1.1 Apresentação dos capítulos . . . . . . . . . . . . . . . . . 3

1.2 Fundamentos de Análise de Sobrevivência . . . . . . . . . . . . . . . 4

1.3 Censuras . . . . . . . . . . . . . . . . . . . 5

1.4 Variáveis explicativas . . . . . . . . . . . . . . . 6

1.5 Tempos de sobrevivência . . . . . . . . . . . . . . . 6

$1.6 \mathrm{O}$ estresse limiar . . . . . . . . . . . . . . . . . . 9

1.7 Modelos de riscos . . . . . . . . . . . . . . . . . . . . 9 9

1.7.1 Modelos de Riscos Proporcionais e de Falha Acelerada . . . . . . . 9

1.7 .2 O Modelo híbrido . . . . . . . . . . . . . . . . . . . 13

1.7 .3 O Modelo híbrido estendido . . . . . . . . . . . . . . . . . 13

1.8 Procedimentos gráficos . . . . . . . . . . . . . . . . . . . . . 14

2 Análise para Testes de Sobrevivência Acelerados sob um Modelo Exponencial com Estresse Limiar $\quad 17$

2.1 Introdução . . . . . . . . . . . . . . . . . . . . . 17

2.2 O modelo exponencial . . . . . . . . . . . . . . . . . . 17

2.3 Métodos de estimação . . . . . . . . . . . . . . . . . . . . . . . . . 19

2.3 .1 Estimação via máxima verossimilhança . . . . . . . . . . . . . . . . 19

2.3.2 Estimação intervalar via "bootstrap" paramétrico e não paramétrico 20

2.3.3 Estimação intervalar via verossimilhança perfilada . . . . . . . . . . 21

2.4 Análise Bayesiana . . . . . . . . . . . . . . . . . . . . . . 21

2.4 .1 O método MCMC . . . . . . . . . . . . . . . . . 22

2.4 .2 O método de Laplace . . . . . . . . . . . . . . . . . . . . . . . . 24

2.5 Aplicações . . . . . . . . . . . . . . . . . . . . . . . . 24

2.5.1 Dados simulados . . . . . . . . . . . . . . . 24 
2.5 .2 Dados reais . . . . . . . . . . . . . . . . . 30

2.6 Conclusões . . . . . . . . . . . . . . . . . . . . . . 34

3 Modelos de Riscos Híbridos PH/AFT com Estresse Limiar 36

3.1 O modelo de riscos híbrido com estresse limiar . . . . . . . . . . . . . 36

3.2 O modelo Weibull . . . . . . . . . . . . . . . . . . . 38

3.3 Métodos de estimação . . . . . . . . . . . . . . . . . . . . . 40

3.3 .1 Estimação via máxima verossimilhança . . . . . . . . . . . . . . . . 40

3.3 .2 Um estudo de simulação . . . . . . . . . . . . . . . . . . . . . . 40

3.3 .3 O método MCMC . . . . . . . . . . . . . . . . . . . 41

3.4 Exemplo numérico . . . . . . . . . . . . . . . . . . . . . 42

3.5 Conclusões . . . . . . . . . . . . . . . . . . . . . . . . 44

4 Um Modelo Geral de Riscos Híbrido com Estresse Limiar 46

4.1 Apresentação do modelo . . . . . . . . . . . . . . . . . . . . 46

4.2 O caso da distribuição gama-generalizada . . . . . . . . . . . . . . . 48

4.3 Métodos de estimação . . . . . . . . . . . . . . . . . . . . . 50

4.3 .1 Estimação via máxima verossimilhança . . . . . . . . . . . . . . 50

4.3 .2 O método MCMC . . . . . . . . . . . . . . . . . . 51

4.4 O caso $\log -\log$ ístico . . . . . . . . . . . . . . . . . . . . . . 52

4.5 Métodos de estimação . . . . . . . . . . . . . . . . . . . . . 54

4.5.1 Máxima verossimilhança . . . . . . . . . . . . . . . . 54

4.5 .2 O método Bayesiano . . . . . . . . . . . . . . . . 55

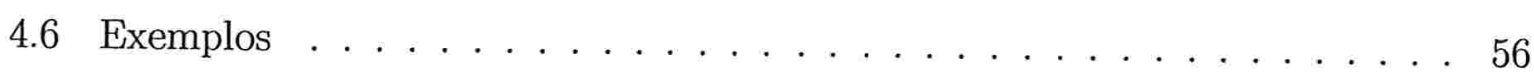

4.6 .1 Dados simulados . . . . . . . . . . . . . . 56

4.6 .2 Dados reais $1 \ldots \ldots \ldots \ldots$. . . . . . . . . . . . . . 58

4.6 .3 Dados reais $2 \ldots \ldots \ldots \ldots$. . . . . . . . . . . . 62

4.7 Conclusões . . . . . . . . . . . . . . . . . . . . . . 64 64

5 A Existência e importância do Estresse Limiar: Testes de Hipóteses 67

5.1 Construção da estatística de teste considerando a distribuição Weibull. . . 68

5.2 Construção da estatística de teste considerando a distribuição Log-logística 71

5.3 Estudos de simulação . . . . . . . . . . . . . . . . . . . . . 72 
5.4 Aplicação a um conjunto de dados reais . . . . . . . . . . . . . . . . . . 74

5.5 A importância do estresse limiar. . . . . . . . . . . . . . . 75

5.6 Conclusões . . . . . . . . . . . . . . . . . . . . 76

6 Modelos de Múltiplos Riscos $\quad 77$

6.1 Introdução . . . . . . . . . . . . . . . . . . . 77

6.2 Determinação empírica da forma da função de risco . . . . . . . . . . . 79

6.3 Modelos de múltiplos riscos na presença de covariáveis . . . . . . . . . . 80

6.4 O caso Weibull perturbado por uma exponencial . . . . . . . . . . . . . . 80

6.4.1 Procedimentos de máxima verossimilhança . . . . . . . . . . . . . 81

6.4 .2 Estimação Bayesiana . . . . . . . . . . . . . . . . . . . 81

6.5 Exemplo: dados simulados . . . . . . . . . . . . . . . . 82

6.6 Conclusões . . . . . . . . . . . . . . . . . . 85

$\begin{array}{ll}\text { Apêndices } & 86\end{array}$

A : Métodos de estimação . . . . . . . . . . . . . . . . . 87

A.1 : Método de máxima verossimilhança . . . . . . . . . . . . . 87

A.2 : Inferências sobre os parâmetros . . . . . . . . . . . . 89

A.3 : Análise Bayesiana para modelos de tempos de vida . . . . . . . . . 90

A.3.1 : Teorema de Bayes . . . . . . . . . . . . . . . . . 90

A.3.3 : Amostrador de Gibs . . . . . . . . . . . . . . . . . . . . 91

A.3.4 : Metropolis-Hastings . . . . . . . . . . . . . . 92

A.3.5 : Método de rejeição adaptativa . . . . . . . . . . . . . 93

B : Diagnóstico de Convergência . . . . . . . . . . . . . . . . 94

B.1 : Diagnóstico de Gelman e Rubin . . . . . . . . . . . . . . 95

B.2 : Diagnóstico de Geweke . . . . . . . . . . . . . . . . 97

B.3 : Diagnóstico de Raftery e Lewis . . . . . . . . . . . . . . . . 97

$\begin{array}{lr}\text { Referências Bibliográficas } & 98\end{array}$ 


\section{Resumo}

Este trabalho tem como objetivo propor modelos de regressão de riscos híbridos que, além de incluir um estresse limiar, contêm como casos particulares os modelos de riscos mais importantes em análise de sobrevivência: os modelos de riscos proporcionais de Cox (PH), os modelos de taxa de falha acelerada (AFT) e o modelo híbrido (Ciampi e EtezadiAmoli, 1985). Para a representação da função de risco base, adotamos uma abordagem paramétrica baseada nos modelos mais importantes utilizados em confiabilidade ou análise de sobrevivência: os modelos log-logístico e gama-generalizado e seus casos particulares, como os modelos Weibull, exponencial, gama e log-normal. Em sua maior parte, este trabalho aborda o problema de estimação das quantidades de interesse, como a média, variância e os intervalos de confiança, para os parâmetros dos modelos, com especial atenção ao estresse limiar. Utilizamos tanto o ponto de vista clássico quanto o Bayesiano. Através de estudos de simulação, mostramos as probabilidades de cobertura dos intervalos de confiança para os parâmetros utilizando a teoria assintótica usual. A metodologia é sempre ilustrada com conjuntos de dados reais. 


\section{Abstract}

In this thesis we propose hibrid hazard regression models which contain as particular cases the most important hazard models in survival analysis: Cox proportional hazard models $(\mathrm{PH})$, the accelerated failure times model (AFT) and the hibrid models (Ciampi e EtezadiAmoli, 1985). Our models also include a threshold stress, whose estimation is of great importance for accelerated failure tests users. In order to represent the hazard function we adopt a parametric approach based on the models often used in survival and reliability analysis: the log-logistic and generalized gamma models and their particular cases, as the Weibull, exponential, gamma and log-normal models. Most of this work is concerned with the estimation problem for the quantities of interest, as the mean, variance and confidence intervals, for the parameters of the models, with special attention to the threshold stress. We consider both classical and Bayesian points of view. We have performed a simulation study in order to examine the coverage probability of the confidence intervals for the parameters based on the usual asymptotic analysis procedures. The methodology is illustrated on real data sets. 


\section{Capítulo 1}

\section{Introdução}

A análise estatística de tempos de vida tem se tornado um tópico de bastante interesse em áreas como engenharia, medicina, demografia e ciências biológicas. Um grande número de trabalhos em Análise de Sobrevivência vem aparecendo nas últimas décadas. Mann et al (1974), Gross e Clark (1975) e Lawless (1982) fazem forte uso de métodos paramétricos para distribuições particulares. Kalbfleisch e Prentice (1980) apresentam com detalhes os modelos de riscos multiplicativos, enquanto Miller (1981) descreve métodos não-paramétricos e semi-paramétricos. Várias aplicações em confiabilidade industrial podem ser vistas em Barlow e Proschan (1975), Nelson (1982) e DePriest e Launer (1983). Elandt-Jonson e Johnson (1980) descrevem aplicações em ciências atuariais e demografia. Miké e Stanley (1982) editaram uma coleção de artigos em Estatística médica que incluem discussões de dados de sobrevivência, e Peto et al. $(1976,1977)$ fornecem aplicações de alguns métodos para a análise de eventos clínicos. Contribuições fundamentais dentro da análise de sobrevivência foram dadas pelos dois artigos mais citados na literatura estatística, a saber, aqueles nos quais foram introduzidos o estimador de Kaplan-Meier para a função de sobrevivência (Kaplan e Meier, 1958) e o modelo de Cox (Cox, 1972).

Geralmente, a expressão análise de sobrevivência diz respeito a dados médicos, enquanto que análise de confiabilidade é mais utilizada para a pesquisa industrial. Ambas consistem de um conjunto de técnicas estatísticas paramétricas e não-paramétricas que são adequadas a dados que medem o tempo até a ocorrência de um evento de interesse, como a falha de um equipamento ou a morte de um paciente em virtude de certa doença, dentre outros. Frequentemente, o evento de interesse não chega a ocorrer para algumas unidades durante o período de observação, dando origem às chamadas censuras, o que torna a análise estatística desses dados distinta das análises usuais. 
Nas últimas décadas, a teoria para a análise de dados de sobrevivência tem sido desenvolvida com o objetivo de estudar a chamada função de risco/sobrevivência. Essa metodologia nos permite determinar quais variáveis afetam a forma das funções de risco e sobrevivência, assim como obter estimativas dessas funções para cada indivíduo ou equipamento. Os dois modelos de riscos considerados os mais importantes nessa área são o modelo introduzido por Cox (Cox, 1972), também conhecido como modelo de riscos proporcionais de Cox, e o modelo de taxa de falha acelerada (Prentice, 1978, e Kalbfleisch e Prentice, 1980) (veja Seção 1.5). Após o "surgimento" do modelo de regressão de Cox, outros modelos surgiram na literatura para a função de risco, os quais generalizam o modelo de regressão de Cox e o de falha acelerada e são denominados modelos híbridos (Ciampi e Etezadi-Amoli, 1985 e 1987). No primeiro de tais trabalhos, os autores usam uma aproximação polinomial para a função de risco base do modelo, enquanto no segundo é utilizada uma aproximação "spline"-quadrática. Em ambos, é empregada apenas uma abordagem não-paramétrica para a estimação da função de risco base. Além disso, embora tais modelos se ajustem a uma grande quantidade de aplicações, eles não se aplicam ao caso em que existe um parâmetro de estresse limiar, ou "threshold stress".

O conceito de estresse limiar tem sido discutido em vários campos de aplicação (Hatch, 1987). Por exemplo, em estudos de toxicologia, modelos que incluem um estresse limiar têm sido propostos (Cox, 1987). Nesses estudos, o estresse limiar pode ser o nível máximo da dose de uma certa substância tóxica, abaixo do qual podemos dizer que não exista efeito de intoxicação em um indivíduo exposto a tal substância. Em aplicações industriais, o estresse limiar corresponde ao nível de estresse ( temperatura, voltagem, etc) abaixo do qual o tempo de vida de um produto pode ser considerado infinito ou muito maior do que o tempo de missão para o qual foi desenvolvido. Para usuários de testes de sobrevivência acelerados (veja Seção 1.6), a determinação do nível de estresse limiar é de fundamental importância (Hirose, 1993).

Motivados pela importância de estimar o estresse limiar em eventos como os descritos acima e pela conveniência de utilizar uma abordagem paramétrica para a estimação da função de risco base, propomos nesta tese modelos alternativos aos tradicionalmente discutidos na literatura de análise de dados de sobrevivência e confiabilidade, aos quais damos o nome de modelos de riscos híbridos com estresse limiar. Em virtude da forma como é definida a função de risco base, os modelos propostos permitem a utilização de várias distribuições importantes empregadas em análise de sobrevivência e confiabilidade. Além 
disso, são capazes de se ajustar a uma grande variedade de dados de sobrevivência.

Em sua maior parte, este trabalho aborda o problema de estimação das quantidades de interesse, como a média, a variância e os intervalos de confiança, sob os pontos de vista clássico e Bayesiano.

Uma breve revisão sobre os conceitos básicos da análise de sobrevivência e dos métodos de estimação para dados de tempo de vida é apresentada ainda neste capítulo.

\subsection{Apresentação dos capítulos}

A seguir, faremos uma breve descrição do conteúdo desta tese, a qual está dividida em sete capítulos relacionados pelo tema comum de modelos de riscos.

No Capítulo 2, desenvolvemos uma metodologia para testes de sobrevivência acelerados, com o principal objetivo de determinar o nível de estresse limiar. A função de risco considerada é a constante, o que nos leva a adotar uma distribuição exponencial para representar os tempos de vida. O modelo é ajustado pelos métodos de máxima verossimilhança, verossimilhança parcial, "bootstrap" paramétrico e não-paramétrico. Do ponto de vista Bayesiano, utilizamos a transformada de Laplace e métodos de MCMC. Parte desse capítulo está condensada nos artigos

A Bayesian Analysis for accelerated Lifetime Tests Under an Exponential Power Law Model with Threshold Stress, Tojeiro, C.A.V., Louzada-Neto,F. and Bolfarine, H. (2004)Journal of Applied Statistics.

A Bayesian Analysis for Hybrid Hazard Models with Threshold Stress, Louzada-Neto, F., Tojeiro, C.A.V. and Bolfarine, H. (2002)- Statistical Modelling in Society.

No Capítulo 3 propomos um modelo de riscos híbrido com estresse limiar que inclui os modelos de falha acelerada (AFT) e o modelo de riscos proporcionais de Cox (PH) como casos particulares. Para tal modelo trabalhamos com a distribuição Weibulll, generalizando assim o modelo proposto no Capítulo 2.

Esse capítulo é baseado nos seguintes trabalhos:

__A Bayesian Analysis for Hybrid Hazard Models with Threshold Stress, LouzadaNeto, F., Tojeiro, C.A.V. and Bolfarine, H. (2002)- Relatório técnico DEs-UFScar: série A: Teoria e Métodos, no. 92.

A Bayesian Analysis for Hybrid Hazard Models with Threshold Stress: the Weibull Case, Louzada-Neto, F., Tojeiro, C.A.V. and Bolfarine, H. (2004)- submetido para publi- 
cação no Statistics.

No Capítulo 4, propomos um modelo ainda mais geral de riscos híbrido com estresse limiar, utilizando agora para a função de risco as distribuições Gama-generalizada e loglogística, o que torna os modelos dos Capítulos 1 e 2 casos particulares do novo modelo.

Esse capítulo faz parte dos trabalhos:

__A General Hybrid Hazard Models with Threshold-Stress, Tojeiro, C.A.V., LouzadaNeto,F. and Bolfarine, H. (2005)- Submetido.

No Capítulo 5, estudamos a existência do parâmetro de estresse limiar através de testes de hipóteses baseados na teoria assintótica de primeira ordem.

Apesar da família gama-generalizada ser bastante útil em vários campos de aplicação, ela não permite o ajuste de funções de risco não monótonas, tais como aquelas em forma de "U" ou as multimodais. Por esta razão, estudamos no Capítulo 6 modelos de riscos múltiplos que utilizam para a função de risco a distribuição bi-Weibull, a qual é adequada para esses casos.

Algumas conclusões e propostas futuras são apresentadas no final de cada capítulo.

\subsection{Fundamentos de Análise de Sobrevivência}

A análise de sobrevivência é uma das áreas da estatística que mais tem crescido nas últimas décadas. Em áreas tais como engenharia, medicina, ciências biológicas e demografia, a análise estatística de tempos de vida tem sido considerada um tópico de grande interesse. Geralmente, a expressão análise de sobrevivência diz respeito a dados médicos, enquanto que análise de confiabilidade é mais utilizada para a pesquisa industrial.

Em análise de sobrevivência, a variável resposta é, geralmente, o tempo até a ocorrência de um evento de interesse, denominado tempo de falha. Em pesquisas médicas, o evento de interesse pode ser o tempo decorrido até o óbito de um paciente, ou o tempo até a ocorrência de uma determinada doença, ou ainda a duração desta. Em pesquisas industriais, o evento de interesse está relacionado à falha de um equipamento, componente ou produto manufaturado. Em ciências sociais, criminalistas estudam o tempo decorrido entre a liberação de presos e a ocorrência de crimes. Em demografia, estuda-se o tempo entre o nascimento e a morte, ou entre o casamento e o divórcio de um indivíduo.

O início da medição do tempo em estudos médicos geralmente coincide com o final do tempo de recrutamento de indivíduos para um determinado estudo experimental, 
enquanto que o tempo final de estudo depende do interesse do pesquisador e da disponibilidade de recursos necessários para a execução da pesquisa. Em pesquisas industriais, o início da medição do tempo corresponde, geralmente, ao momento em que o produto em estudo é colocado em funcionamento para o teste, enquanto que o tempo final coincide com a falha do equipamento ou com o final pré-fixado do estudo.

\subsection{Censuras}

A principal característica de dados de sobrevivência é a presença de censuras, as quais consistem na observação parcial da resposta, tendo em vista a possibilidade de que o evento de interesse não ocorra em um tempo pré-estabelecido. Como exemplos de causas de censuras, podemos ter o abandono de um paciente antes do término do estudo, ou a sua morte por causa diferente da estudada, ou ainda a não ocorrência da falha de um equipamento em tempo pré-determinado, etc. Sem a presença de censuras, as técnicas estatísticas clássicas, tais como a análise de regressão e o planejamento de experimentos, poderiam ser utilizadas na análise desse tipo de dados. Por exemplo, suponhamos que o evento de interesse seja comparar o tempo médio de vida de três grupos de pacientes. Caso não houvessem censuras, poderíamos empregar técnicas usuais de análise de variância para fazer tal comparação. Entretanto, na presença de censuras tais técnicas não poderiam ser utilizadas, pois elas necessitam de todos os tempos de falha.

Existem três tipos de censura importantes em análise de sobrevivência, denominadas censura à direita, censura à esquerda e censura intervalar. Na censura à direita, o tempo de sobrevivência não é observado, mas sabe-se que excedeu um certo valor. Na censura à esquerda, sabe-se apenas que o tempo de sobrevivência é menor do que um certo valor, enquanto que, na censura intervalar, o evento ocorre entre dois instantes conhecidos. Dentre as censuras à direita, podemos ter os casos de censura tipo I, censura tipo II e censura aleatória. Na censura tipo I, a duração do experimento é pré-fixada, de tal modo que, caso a falha não ocorra antes ou até o tempo pré-fixado, os tempos de falha são censurados. Na censura tipo II, o número de observações censuradas é fixo e conhecido a priori, ou seja, o estudo termina após ter ocorrido o evento de interesse em um número pré-estabelecido de indivíduos ou equipamentos. Outros tipos de censura podem ser encontrados em Collet (1994).

Nesta tese, a única forma de censura considerada será a censura à direita, embora 
os princípios utilizados para a análise de máxima verossimilhança não dependam desta restrição.

\subsection{Variáveis explicativas}

Além do tempo de sobrevivência e da variável indicadora de censura, também podemos observar, nos dados, variáveis que representam a heterogeneidade existente na população, tais como idade, sexo, dentre outras, assim como, no caso de dados médicos, possíveis tratamentos aos quais os indivíduos em estudo sejam submetidos. Tais variáveis são conhecidas como variáveis explicativas ou covariáveis.

Um dos objetivos da análise de sobrevivência é estudar a relação entre o tempo de sobrevivência e algumas das covariáveis de interesse. Do ponto de vista estatístico temos, disponíveis para a análise, variáveis relacionadas ao tempo de sobrevivência, uma variável indicadora de censura e um vetor de variáveis explicativas. Uma dificuldade que pode ocorrer em análise de sobrevivência e confiabilidade é a existência de covariáveis que dependem do tempo, ou seja, variáveis explicativas cujos valores ao final do estudo podem não ser os mesmos que em seu início. Como exemplo, podemos ter um experimento em que a dose de um determinado medicamento é modificada ao longo do estudo, em virtude de efeitos colaterais provocados pelo mesmo no paciente.

\subsection{Tempos de sobrevivência}

Para expressar a distribuição de uma variável aleatória não-negativa $T$ que representa o tempo de falha ou sobrevivência, várias funções matemáticas equivalentes podem ser utilizadas. Dentre elas, podemos considerar a função de sobrevivência, a função de densidade de probabilidade, a distribuição cumulativa e a função de risco.

A função de sobrevivência ou função de taxa de falha (ou risco) $S$ é definida como a probabilidade de um indivíduo sobreviver até um determinado tempo $t$. Em termos probabilísticos,

$$
S(t)=P(T \geq t)=1-F_{T}(t)
$$

onde $F_{T}(t)=P(T \leq t)$ é a função de distribuição (cumulativa) de $T$. A função $S(t)$ é 
não-crescente e goza das propriedades de que $S(0)=1$ e $\lim _{t \rightarrow \infty} S(t)=0$.

A função de densidade de probabilidade de $T$ (para distribuições contínuas) é definida como o limite do quociente da probabilidade de que a falha ocorra no intervalo de tempo $(t, t+\Delta t)$ por $\Delta t$ quando $\Delta t$ tende a zero, ou seja,

$$
f(t)=\lim _{\Delta t \rightarrow 0} \frac{P(t \leq T \leq t+\Delta t)}{\Delta t}=-S^{\prime}(t)=\frac{d F_{T}(t)}{d t}
$$

A função $f(t)$ possui duas propriedades básicas:

$$
f(t) \geq 0 \quad e \int_{0}^{\infty} f(t) d t=1
$$

Observe que a função de sobrevivência pode ser representada em termos de $f(t)$ por $S(t)=\int_{t}^{\infty} f(x) d x$. Na prática, quando não há observações censuradas, a função de sobrevivência é estimada pela proporção de indivíduos que sobreviveram além do instante $t$ e o total de indivíduos no estudo. Entretanto, na presença de censuras, estimadores mais gerais devem ser considerados.

A função de risco ou taxa de falha $h(t)$, muito útil para descrever a distribuição do tempo de vida de pacientes ou equipamentos, é definida como o limite, quando $\Delta t$ tende a zero, do quociente por $\Delta t$ da probabilidade de um indivíduo falecer no intervalo de tempo $(t, t+\Delta t)$, dado que o mesmo tenha sobrevivido até o tempo $t$ (Cox e Oakes, 1984), isto é,

$$
h(t)=\lim _{\Delta t \rightarrow 0} \frac{P(t \leq T \leq t+\Delta t / T \geq t)}{\Delta t}
$$

Decorre da definição de probabilidade condicional que

$$
h(t)=\frac{f(t)}{S(t)}=\frac{-S^{\prime}(t)}{S(t)}=-\frac{d}{d t}(\log S(t)) .
$$

Uma vez que $S(0)=1$, obtemos

$$
S(t)=\exp \left(-\int_{0}^{t} h(u) d u\right)
$$

$\log 0$

$$
f(t)=h(t) \exp \left(-\int_{0}^{t} h(u) d u\right)
$$


A expressão (1.5) é muito importante quando desenvolvemos os procedimentos de estimação somente sobre a função de risco.

A função de risco descreve a forma como a probabilidade instantânea de falha muda com o tempo. Ela também é conhecida como taxa instantânea de falha, força de mortalidade ou taxa de mortalidade condicional. Além disso, classes especiais de distribuições de tempos de vida podem ser caracterizadas pelo comportamento da função de risco, a qual pode ser crescente, decrescente, constante ou mesmo não monótona. Funções de risco crescentes ocorrem, por exemplo, quando pacientes com uma determinada doença crônica não respondem a um tratamento, enquanto que o caso de pacientes que respondem ao tratamento corresponde a curvas decrescentes. A função de risco constante pode ser apropriada para representar o risco de falecimento de indivíduos entre 20 e 30 anos, cujas principais causas de morte são acidentes. Em geral, sabe-se que a taxa de falha para o tempo de vida de seres humanos é uma combinação dos três tipos de curvas. Assim, uma função de risco em forma de "U" pode ser apropriada para representar o comportamento do risco de uma população humana que, após um certo período de grande taxa de falha nos primeiros anos de vida devida a possíveis causas genéticas e doenças infantis, decai até se estabilizar na idade adulta, a partir da qual passa a crescer.

Algumas distribuições usuais de tempos de sobrevivência são a exponencial, Weibull, log-normal, log-logística e gama-generalizada. Esta última é composta por dois parâmetros de forma e um de escala, assumindo assim uma imensa variedade de formas. As distribuições comumente utilizadas para modelagem de dados de sobrevivência são casos especias dessa distribuição. A distribuição exponencial acomoda funções de risco constantes, enquanto que, para funções de risco monotônicas, é mais apropriado utilizar uma distribuição Weibull (Lawless, 1982). As distribuições log-logística e log-normal acomodam funções de risco unimodais (Kalbfleisch e Prentece, 1980). Para as funções de risco multimodais ou em forma de "U", Louzada-Neto (1999) propôs um modelo de riscos múltiplos que acomoda tais formas de funções de risco.

Uma outra função importante é a chamada função de risco acumulada, dada por

$$
H(t)=-\log S(t)=\int_{0}^{t} h(u) d u
$$

em que a segunda igualdade decorre de (1.5). 


\subsection{O estresse limiar}

O principal interesse na área de confiabilidade de produtos é fazer inferências sobre a taxa de falha, ou funções da mesma, em condições usuais, baseadas em dados obtidos em condições aceleradas. Em geral, tem-se interesse no tempo médio de sobrevivência de componentes e no nível de estresse limiar (threshold stress). O conceito de estresse limiar tem sido discutido em vários campos de aplicação (Hatch, 1987). Por exemplo, em estudos de toxicologia, modelos que incluem um estresse limiar têm sido propostos (Cox, 1987). Nesses estudos, o estresse limiar pode ser o nível máximo da dose de uma certa substância tóxica, abaixo do qual podemos dizer que não exista efeito de intoxicação em um indivíduo exposto a tal substância. Em aplicações industriais, o estresse limiar corresponde ao nível máximo (respectivamente, mínimo) de estresse ( temperatura, voltagem, etc) abaixo do qual (respectivamente, acima do qual) o tempo de vida de um produto pode ser considerado infinito ou muito maior do que o tempo de missão para o qual foi desenvolvido. Ou seja, em termos formais, podemos definir o estresse limiar $\omega_{0}$ como: $P\left[T>t \mid Z_{i}-\omega_{0}>0\right]=1$.Para usuários de AFT, a determinação do nível de estresse limiar é de fundamental importância.

Ignorar o parâmetro de estresse limiar, quando este existe, pode nos levar a um erro de estimação como mostrado na Seção 5.5 do capítulo 5 .

\subsection{Modelos de riscos}

\subsubsection{Modelos de Riscos Proporcionais e de Falha Acelerada}

Em análise de sobrevivência existem duas classes importantes de modelos propostas na literatura: os modelos paramétricos e os modelos semi-paramétricos. Os primeiros, também conhecidos como modelos de falha acelerada (Prentice, 1978), são mais eficientes porém menos flexíveis que os modelos semi-paramétricos ou modelos de regressão de Cox (Cox, 1972). Tais modelos são capazes de ajustar uma grande quantidade de aplicações. O modelo de riscos proporcionais, além de ser bastante flexível, permite incoporar covariáveis dependentes do tempo, as quais ocorrem com frequência em várias áreas.

Considere um estudo clínico controlado que consiste na comparação de tempos de sobrevivência ajustados por covariáveis. Seja $h(t / z)$ a função de risco no tempo $t$ para um indivíduo com um vetor de covariáveis $z$. O modelo de riscos proporcionais é dado 
por

$$
h(t / z)=g\left(\alpha^{\prime} z\right) h_{0}(t)
$$

onde $g$ (.) é uma função monótona cuja forma paramétrica é conhecida, a qual é igual a um quando todos os seus argumentos valem zero, $h_{0}(t)$ é a função de risco base, não negativa no tempo, $z$ representa o vetor de covariáveis e $\alpha^{\prime}$ é o vetor de coeficientes do modelo a ser estimado. Várias formas funcionais podem ser empregadas para $g($.$) , entretanto$ a candidata mais natural é a exponencial, a qual garante, em particular, que $h(t)$ seja sempre positiva.

O modelo (1.8) admite que o vetor de covariáveis $z$ tenha um efeito multiplicativo na função de risco, o que implica na proporcionalidade entre as funções de risco de diferentes níveis de covariáveis. Ou seja, a razão das taxas de falha de dois indivíduos diferentes é constante ao longo do tempo, não permitindo que as funções de risco se cruzem. Esta é uma grande limitação dos modelos de riscos proporcionais, uma vez que, na prática, não é difícil encontrarmos situações em que ocorrem funções de risco não proporcionais (Ciampi e Etezadi-Amoli, 1985).

Para acomodar situações nas quais o modelo de riscos proporcionais (1.8) não é adequado, temos o modelo de falha acelerada

$$
h(t / z)=g\left(\alpha^{\prime} z\right) h_{0}\left(g\left(\alpha^{\prime} z\right) t\right) .
$$

A vantagem deste modelo é permitir funções de risco que se cruzam, uma vez que $z$ tem efeito multiplicativo não somente na função de risco mas também em $t$. Desta forma, $z$ afeta o tempo de sobrevivência causando deformação, ou seja, aceleração ou desaceleração, na escala do tempo (Etezadi-Amoli e Ciampi, 1987).

\section{Testes de sobrevivência acelerados e relações estresse-resposta gerais}

Atualmente, a utilização de testes de sobrevivência acelerados vem crescendo com a necessidade de materiais ou sistemas que exigem alta confiabilidade. Testes de sobrevivência acelerados são testes em que a resposta de interesse é o tempo até a ocorrência de falha de um determinado componente. Eles são utilizados para caracterizar, de forma rápida, informações a respeito da distribuição do tempo de falha ou outras propriedades de durabilidade de materiais ou componentes em estudo. A vantagem desses testes é minimizar 
o tempo e o custo do experimento para a obtenção das estimativas de interesse.

Vários métodos de aceleração podem ser considerados. Por exemplo, aceleração por alta taxa de uso ou por níveis de estresse mais altos e degradação. A carga de estresse pode ser aplicada de várias formas: constante, escada, progressiva, cíclica e aleatória (Nelson, 1990).

A variável estresse é aquela que, quando utilizada em níveis diferentes daqueles sob condições normais de uso, acelera o tempo de falha do componente. O teste consiste em realizar o experimento de modo que as unidades sob estudo sejam submetidas a níveis da variável ( temperatura, voltagem, pressão, umidade, choque, etc) mais altos (ou baixos) que o usual.

A partir do conhecimento prévio a respeito da variação do comportamento de falha nas condições usuais e através dos testes de sobrevivência conduzidos sob condições aceleradas, podemos fazer inferências sobre as características da distribuição de vida correspondentes às condições normais de uso do produto.

Os modelos de testes de sobrevivência acelerados envolvem dois componentes: a distribuição do tempo de vida das unidades e a relação estresse-resposta que modela o efeito que as variáveis ou covariáveis terão na distribuição do tempo de falha.

De um modo geral, o conhecimento físico mostra que, frequentemente, pode existir uma ou mais relações entre os parâmetros da distribuição de falha e as condições do teste. O uso desses modelos ou relações é amplamente discutido na literatura, particularmente por pesquisadores que tratam de mecanismos que induzem às falhas. Vários modelos são utilizados na área industrial; para uma descrição completa veja Mann, Shaffer e Singpurwalla (1974). Dentre os modelos mais utilizados, podemos citar o modelo de taxa de reação de Arrhenius,

$$
\lambda_{i}=\exp \left\{\alpha-\frac{\beta}{x_{i}}\right\}
$$

e o modelo de Eyring para uma variável estresse,

$$
\lambda_{i}=x_{i} \exp \left\{\alpha-\frac{\beta}{x_{i}}\right\}
$$

onde $\lambda_{i}$ é o parâmetro da distribuição de falha, $x_{i}$ é o i-ésimo nível da variável estresse (de temperatura, pressão, etc) e $\alpha$ e $\beta$ são parâmetros tais que $\alpha>0$ e $-\infty<\beta<\infty$. Estes dois modelos têm grande aplicação em componentes eletrônicos, como por exemplo 
em semi-condutores, e expressam a taxa do tempo de degradação de algum parâmetro como função da temperatura. O modelo de lei da potência inversa,

$$
\lambda_{i}^{-1}=\frac{\alpha}{x_{i}^{\beta}}
$$

é baseado em conceitos de mecânica estatística, tais como energia de ativação e teoria cinética. Tal modelo é muito usado na indústria e engenharia, com grande aplicação em lâmpadas incandescentes, fadiga de materiais e capacitores dielétricos. Ele expressa o aumento da taxa de falha $\lambda$ com a $\beta$-ésima potência da voltagem $x$ aplicada ao componente.

Nesta tese definimos uma relação estresse-resposta (SRR) bastante geral que inclue um estresse limiar $\omega_{0}$. Para o caso em que $\omega_{0}$ corresponde ao nível de estresse máximo (por exemplo, voltagem) abaixo do qual o tempo de vida de um produto pode ser considerado infinito ou muito maior do que o tempo de missão para o qual foi desenvolvido, definimos uma relação estresse-resposta (SRR) com estresse limiar $\omega_{0}$ bastante geral dada por

$$
\lambda_{i}=\exp \left\{-\left(w_{i}+\beta_{0}+\beta_{1}\left(Z_{i}-\omega_{0}\right)\right)\right\}
$$

Observe que, pela definição de estresse limiar, podemos supor que $Z_{i}>\omega_{0}$, para todo $i$. Por outro lado se o estresse limiar corresponde, ao nível mínimo (por exemplo, temperatura) acima do qual o tempo de vida de um produto pode ser considerado infinito, a relação SRR é dada por

$$
\lambda_{i}=\exp \left\{-\left(w_{i}+\beta_{0}+\beta_{1}\left(\omega_{0}-Z_{i}\right)\right)\right\}
$$

onde agora temos $\omega_{0}>Z_{i}$, para todo $i$.

Essas relações incluem várias SRR's usadas em confiabilidade como casos particulares:

1. Quando $\omega_{0}=0$ em (1.10) temos a SRR geral definida em (Achcar e Louzada-Neto, 1992).

2. O modelo de lei da potência inversa com estresse limiar é obtido para $w_{i}=0$, $\left(Z_{i}-\omega_{0}\right)=-\log \left(x_{i}-\omega_{0}\right), \beta_{0}=\log \alpha$ e $\beta_{1}=\beta$, adicionalmente se $\omega_{0}=0$, temos o modelo de lei da potência inversa.

3. O modelo de Arrhenius com estresse limiar é obtido fazendo $w_{i}=0,\left(Z_{i}-\omega_{0}\right)=$ $1 /\left(x_{i}-\omega_{0}\right), \beta_{0}=-\alpha$ e $\beta_{1}=\beta$, consequentemente para $\omega_{0}=0$, temos o modelo de Arrhenius. 
4. Caso $w_{i}=-\log \left(x_{i}\right),\left(Z_{i}-\omega_{0}\right)=1 /\left(x_{i}-\omega_{0}\right), \beta_{0}=-\alpha$ e $\beta_{1}=\beta$, temos o modelo de Eyring com estresse limiar. Tomando $\omega_{0}=0$, temos o modelo de Eyring.

Neste trabalho, consideraremos sempre a relação SRR (1.10) com o modelo de lei da potência inversa correspondente ao caso de um máximo estresse limiar, como descrita no ítem (2) acima, ou seja,

$$
\lambda_{i}=\exp \left\{-\beta_{0}+\beta_{1} \log \left(x_{i}-\omega_{0}\right)\right\} .
$$

Embora somente tal SRR (com o modelo de lei da potência inversa) seja considerada aqui, a metodologia é geral e pode ser aplicada para outras relações estresse-resposta.

\subsubsection{O Modelo híbrido}

Após o "surgimento" do modelo de regressão de Cox, outros modelos surgiram na literatura para a função de risco, os quais são denominados modelos híbridos para a função de risco e generalizam o modelo de regressão de Cox e o de falha acelerada. Com o intuito de acomodar ambos os modelos em uma família híbrida, (Ciampi e Etezadi-Amoli, 1985) propuseram um modelo de riscos híbrido dado por

$$
h(t / z)=g_{1}\left(\alpha_{1}^{\prime} z\right) h_{0}\left(g_{2}\left(\alpha_{2}^{\prime} z\right) t\right)
$$

em que $\alpha_{1}$ e $\alpha_{2}$ são vetores de coeficientes. O modelo (1.13) generaliza os modelos (1.8) e (1.9). Para $\alpha_{2}=0$ temos o modelo de riscos proporcionais e para $g_{1}()=.g_{2}$ (.) e $\alpha_{1}=\alpha_{2}$ o modelo de falha acelerada.

\subsubsection{O Modelo híbrido estendido}

Os modelos descritos anteriormente são bastante utilizados na análise de dados de sobrevivência. Entretanto, tais modelos não se aplicam ao caso em que o parâmetro de forma da distribuição depende de diferentes níveis de covariáveis. Este fato é caracterizado por uma possível diferença na forma das distribuições de sobrevivência, relacionadas a diferentes níveis de covariáveis. Quando utilizamos os modelos (1.8),(1.9) e (1.13), estamos sempre modelando o parâmetro de escala, enquanto que o parâmetro de forma é suposto constante para todos os níveis das covariáveis, ou é livre, no sentido de que temos um parâmetro de forma para cada nível. 
Louzada-Neto (1997) propôs um modelo de riscos híbrido estendido, o qual permite que o parâmetro de forma dependa de covariáveis por meio de uma função "heterocedástica". Esse modelo é dado por

$$
h(t / z)=g\left(\alpha_{1}^{\prime} z\right) g\left(\gamma^{\prime} z\right)\left[g\left(\alpha_{1}^{\prime} z\right)\right]^{g\left(\gamma^{\prime} z\right)-1} h_{0}\left[g\left(\alpha_{2}^{\prime} z\right) t\right]^{g\left(\gamma^{\prime} z\right)},
$$

em que $\alpha_{1}, \alpha_{2}$ e $\gamma$ são vetores de coeficientes de regressão desconhecidos e $h_{0}$ (.) é a função de risco base. A função $g\left(\gamma^{\prime} z\right)$ descreve a relação "heterocedástica" entre o parâmetro de forma e as covariáveis. Como casos particulares temos: para $\gamma=0$ o modelo de risco híbrido (1.13), para $\gamma=0$ e $\alpha_{2}=0$ o modelo de riscos proporcionais (1.8) e para $\gamma=0$ e $\alpha_{1}=\alpha_{2}$ o modelo de falha acelerada (1.9). Versões estendidas dos modelos (1.8), (1.9) são obtidas quando $\gamma \neq 0, \alpha_{2}=0$ e quando $\gamma \neq 0$ e $\alpha_{1}=\alpha_{2}$.

\subsection{Procedimentos gráficos}

Em análise de sobrevivência, uma forma de avaliar se determinada distribuição é adequada para representar certos dados de tempos de vida é por meio de diagnósticos gráficos. Gráficos de $\hat{S}(t)$, a função de sobrevivência estimada (Kaplan-Meier), ou $\hat{H}(t)=-\log \hat{S}(t)$, a função de risco acumulado, fornecem informações sobre a forma da distribuição que melhor se adequa aos dados. Eles têm sido usados como uma maneira de ajudar a encontrar a forma da função de risco. A idéia básica é a de construir certos gráficos que devem ser aproximadamente lineares caso a família de modelos proposta seja adequada.

Suponha, por exemplo, que a possibilidade de uma distribuição exponencial seja considerada. A função de sobrevivência para esta é dada por $\log S(t)=-\lambda t$ (veja Capítulo 2) e, portanto, o gráfico de $\log \hat{S}(t)$ por $t$ deve ser linear e passar pela origem.

Por outro lado, a função de sobrevivência de um modelo Weibull é dada por $S(t)=$ $\exp \left[-(\lambda t)^{\phi}\right]$ (veja Capítulo 3), portanto $\log (-\log S(t))=\phi \log t+\phi \log \lambda$. Assim, o gráfico de $\log (-\log \hat{S}(t))$ versus $\log t$ deve ser aproximadamente linear com inclinação $\phi$ se $S(t)$ é bem ajustada por um modelo Weibull. Em particular, como a distribuição de Weibull reduz-se à exponencial quando $\phi=1$, o gráfico de $\log (-\log \hat{S}(t))$ versus $\log t$ deve ser aproximadamente linear com inclinação 1 (isto é, fazer um ângulo de $45^{\circ}$ com o eixo das abcissas), caso $S(t)$ fosse bem ajustada por um modelo exponencial.

Da mesma forma, como $S(t)=1 /\left(1+(\lambda t)^{\phi}\right)$, ou seja, log $\left[(\hat{S}(t))^{-1}-1\right]=\phi \log t+$ $\phi \log \lambda$, para uma distribuição log-logística (veja Capítulo 4), teríamos uma indicação de 
que $S(t)$ estaria bem ajustada por um modelo log-logístico caso fosse linear o gráfico de $\log \left[(\hat{S}(t))^{-1}-1\right]$ versus $\log t$.

Esses gráficos também são utilizados para avaliar se o parâmetro de forma de uma certa distribuição depende de covariáveis, ou se este pode ser considerado constante para vários níveis da covariável, como por exemplo, níveis de estresse. Em situações de mesma variância para todos os níveis de estresse, espera-se observar retas paralelas (Nelson, 1990). Caso retas paralelas não sejam observadas, temos uma indicação de que a suposição de que o parâmetro de forma seja constante não é apropriada. Para maiores detalhes veja Lawless (1992). Como exemplo, considere os dados obtidos de testes de vida acelerados na Figura 1.1. Em 1.1-a temos dados gerados de uma distribuição exponencial com 3 níveis de estresse $x_{i}=5,10,15$ com 100 observações não censuradas em cada nível. Podemos observar, nesse gráfico, relações lineares e paralelas fazendo um ângulo de $45^{\circ} \mathrm{com}$ o eixo das abcissas. Em 1.1-b consideramos os dados de 70 fibras Kevlar 49 apresentados na Tabela 2 de Smith (1991), com 3 níveis de estresse, $x_{i}=23.4$, 25.5 e 27,6 MPa. Observe que temos relações aproximadamente lineares e paralelas, o que indica que um modelo Weibull com parâmetro de forma constante é apropriado para esses dados. Na Figura 1.1-c temos dados gerados de uma distribuição log-logística com 3 níveis de estresse $x_{i}=5,10,15$. O gráfico de $\log \left[\hat{S}(t)^{-1}-1\right]$ versus $\log t$ é aproximadamente linear com retas paralelas, indicando um bom ajuste por tal modelo, com parâmetro de forma constante. Finalmente, em 1.1-d temos os dados apresentados na Tabela 4.1 de Hirose (1993). Observe que neste caso as retas correspondentes aos vários níveis da covariável não são paralelas, indicando que o parâmetro de forma não é constante (veja, Louzada-Neto, 1997). 


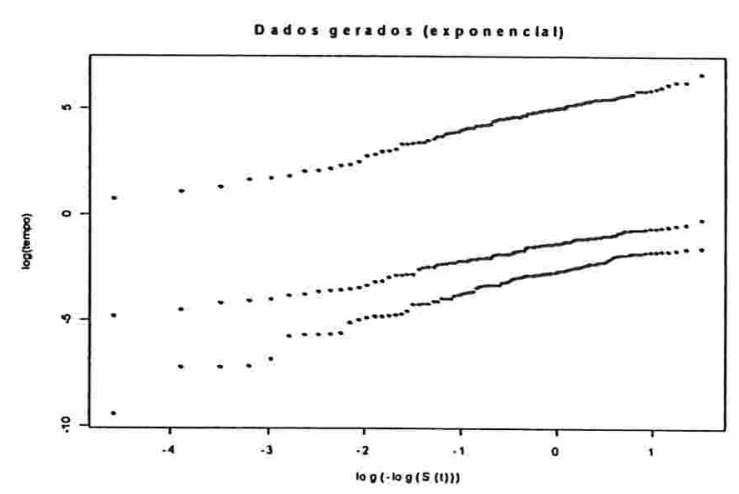

(a)

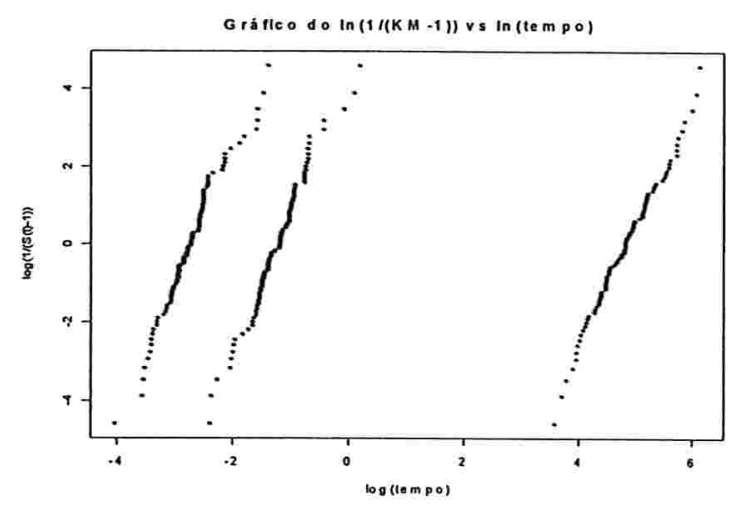

(c)

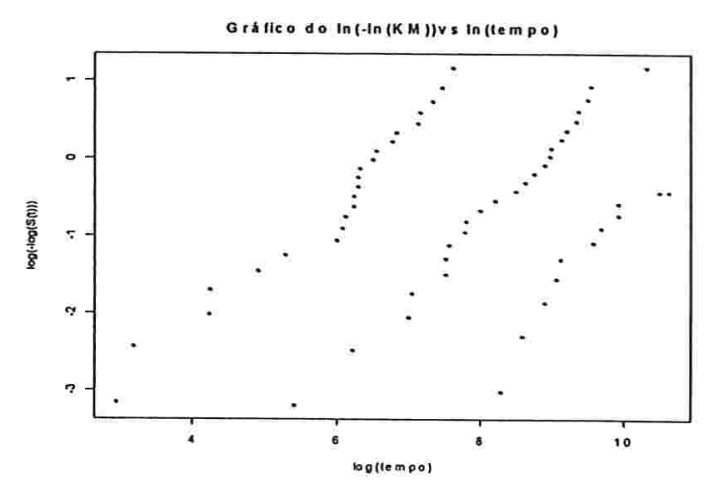

(b)

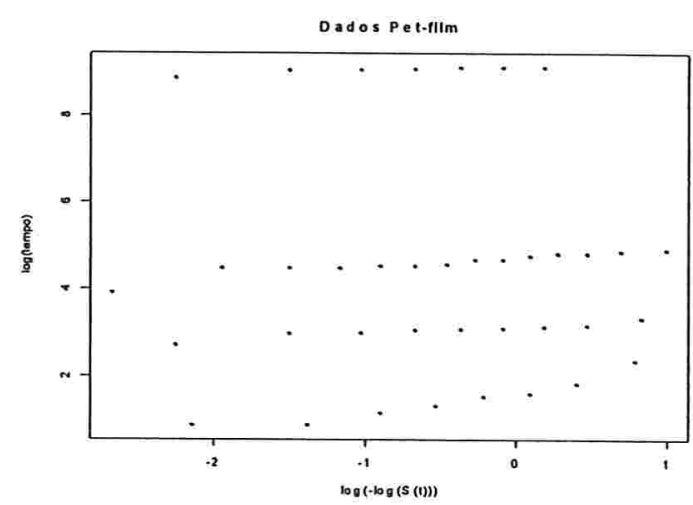

(d)

Figura 1.1: Gráficos das funções de sobrevivência estimada para diferentes distribuições. 


\section{Capítulo 2}

\section{Análise para Testes de Sobrevivência}

\section{Acelerados sob um Modelo}

\section{Exponencial com Estresse Limiar}

\subsection{Introdução}

Neste capítulo, apresentamos metodologias para a modelagem de testes de sobrevivência acelerados sob um modelo de lei da potência inversa com um estresse limiar para a relação estresse-resposta. Para expressar o comportamento dos tempos de vida usamos uma distribuição exponencial. Nosso principal objetivo é determinar o nível de estresse limiar, abaixo do qual a vida de um produto pode ser considerada infinita, ou muito maior do que o tempo de missão para o qual foi desenvolvido. Para efeito de comparação, a estimação dos parâmetros é feita utilizando-se análises clássicas baseadas na normalidade assintótica dos parâmetros envolvidos e métodos de simulação "bootstrap" paramétrico e não paramétrico, além do método de verossimilhança parcial. Do ponto de vista Bayesiano, utilizamos técnicas de MCMC e transformadas de Laplace.

\subsection{O modelo exponencial}

A distribuição exponencial é um dos modelos probabilísticos mais simples usados para descrever o tempo de falha, pois ela apresenta apenas um parâmetro e é a única que se caracteriza por ter uma função de taxa de falha constante. Ela tem sido usada extensivamente na literatura de análise de sobrevivência e confiabilidade, assim como em 
estudos sobre o tempo de vida de ítens manufaturados (Davis, 1952) e em pesquisas de sobrevivência ou tempo de remissão de doenças (Feigl e Zelen, 1965).

A função de densidade de probabilidade para a variável aleatória $T$, tempo de falha, com distribuição exponencial, é dada por:

$$
f(t, \lambda)=\lambda \exp (-\lambda t)
$$

em que $\lambda>0$ é o parâmetro desconhecido que representa a taxa de falha constante. As funções de sobrevivência e taxa de falha são dadas, respectivamente, por:

$$
S(t, \lambda)=\exp (-\lambda t)
$$

e

$$
h(t, \lambda)=\lambda
$$

para $t \geq 0$. Em virtude da função de risco ser constante, a distribuição exponencial é adequada em situações em que a morte de um indivíduo (ou a falha de um equipamento) independe da idade (ou tempo de uso) do mesmo. Isto significa, por exemplo, que um equipamento com 1000 horas de funcionamento tem o mesmo risco de falha que outro recém fabricado. Esta propriedade é conhecida como falta de memória da distribuição exponencial.

Função de Sobrevivência Exponencial

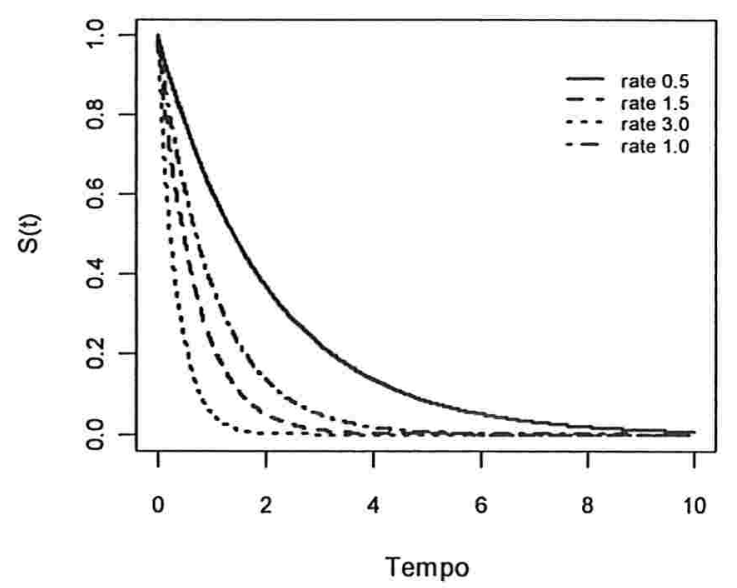

Função de Risco Exponencial

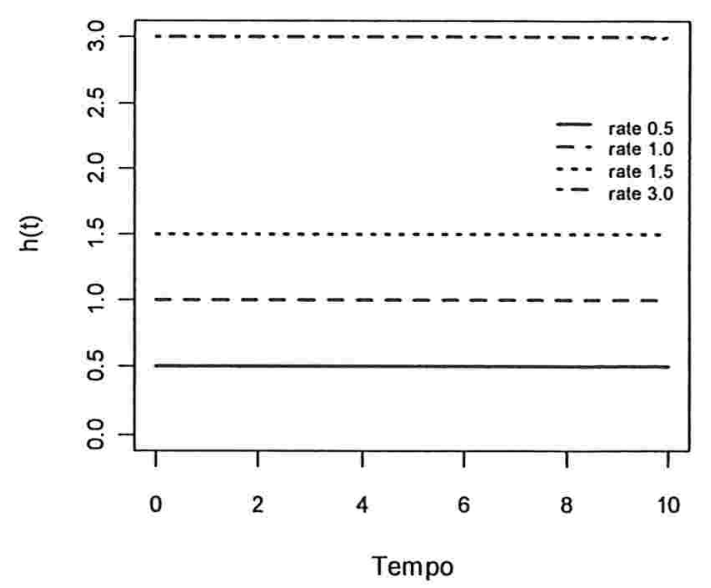

Figura 2.1: Gráfico das funções de sobrevivência e risco da distribuição exponencial para diferentes valores de $\lambda$ 


\subsection{Métodos de estimação}

\subsubsection{Estimação via máxima verossimilhança}

Como mencionado no Capítulo 1, utilizaremos para a taxa de falha a relação estresseresposta geral (1.12) com o modelo de lei da potência inversa com estresse limiar, definido por:

$$
\lambda_{i}=\exp \left(-\beta_{0}+\beta_{1} \log \left(x_{i}-\omega_{0}\right)\right)
$$

onde $-\infty<\beta_{0}, \beta_{1}<\infty$ são parâmetros desconhecidos e $x_{i}$ é o $i$-ésimo nível de estresse. Portanto, a função de log-verossimilhança (6.17) é dada por

$$
\begin{gathered}
l=\log L\left(\beta_{0}, \beta_{1}, \omega_{0}\right) \alpha \sum_{i=1}^{m} \sum_{j=1}^{n_{i}}\left\{\delta_{i j}\left(-\beta_{0}+\beta_{1} \log \left(x_{i}-\omega_{0}\right)\right)-\right. \\
\left.\left[\exp \left(-\beta_{0}+\beta_{1} \log \left(x_{i}-\omega_{0}\right)\right)\right] t_{i j}\right\}
\end{gathered}
$$

Consequentemente,

$$
\frac{\partial l}{\partial \beta_{0}}=\sum_{i=1}^{m} \sum_{j=1}^{n_{i}}\left\{-\delta_{i j}+\left[\exp \left(-\beta_{0}+\beta_{1} \log \left(x_{i}-\omega_{0}\right)\right)\right] t_{i j}\right\}
$$

$$
\frac{\partial l}{\partial \beta_{1}}=\sum_{i=1}^{m} \sum_{j=1}^{n_{i}} \delta_{i j}\left(\log \left(x_{i}-\omega_{0}\right)\right)-t_{i j} \log \left(x_{i}-\omega_{0}\right)\left[\exp \left(-\beta_{0}+\beta_{1} \log \left(x_{i}-\omega_{0}\right)\right)\right]
$$

$\mathrm{e}$

$$
\frac{\partial l}{\partial \omega_{0}}=\sum_{i=1}^{m} \sum_{j=1}^{n_{i}}\left\{\delta_{i j}\left(-\beta_{1} /\left(x_{i}-\omega_{0}\right)\right)+t_{i j} \beta_{1} /\left(x_{i}-\omega_{0}\right)\left[\exp \left(-\beta_{0}+\beta_{1} \log \left(x_{i}-\omega_{0}\right)\right)\right] t_{i j}\right\}
$$

Como podemos observar, as derivadas de primeira ordem são não lineares. Assim, as estimativas do vetor de parâmetros $\left(\beta_{0}, \beta_{1}, \omega_{0}\right)^{t}$, através da maximização direta da função de log-verossimilhança (2.5), podem ser obtidas via métodos de resolução de sistemas não lineares, como, por exemplo, o método conhecido como quasi-Newton (Hartmann, 1994). 
Como descrito no Apêndice A, as inferências são baseadas na normalidade assintótica dos estimadores de máxima verossimilhança (Cox e Hinkley, 1974), isto é:

$$
\left(\hat{\beta}_{0}, \hat{\beta}_{1}, \hat{\omega}_{0}\right) \sim N\left(\left(\beta_{0}, \beta_{1}, \omega_{0}\right), I^{-1}\left(\hat{\beta}_{0}, \hat{\beta}_{1}, \hat{\omega}_{0}\right)\right)
$$

onde $I\left(\hat{\beta}_{0}, \hat{\beta}_{1}, \hat{\omega}_{0}\right)$ é a matriz de informação de Fischer calculada nos EMV's de $\left(\beta_{0}, \beta_{1}, \omega_{0}\right)$.

A utilização de propriedades assintóticas para fazer inferências sobre os parâmetros é direcionada pelo tamanho da amostra, a qual deve ser suficientemente grande. Uma vez que, em análise de sobrevivência e confiabilidade, temos em geral amostras pequenas ou moderadas, outras técnicas devem ser utilizadas. Dentre as mais conhecidas, temos as técnicas de "bootstrap" paramétrico e não paramétrico, verossimilhança perfilada, e verossimilhança perfilada corrigida via "bootstrap" paramétrico e não paramétrico.

\subsubsection{Estimação intervalar via "bootstrap" paramétrico e não paramétrico}

O termo bootstrap é utilizado para denominar uma técnica de simulação que visa a obtenção de estimativas intervalares empíricas para os estimadores dos parâmetros de interesse através da reamostragem do conjunto de dados original (Efron, 1979). A idéia principal do método é tratar os dados como se fossem a população, e retirar amostras (com reposição) dos dados como se fossem amostragem da população. Esse procedimento é repetido um grande número de vezes (digamos $R$ ), em cada uma das quais calculase a quantidade de interesse. Os $R$ valores obtidos são então usados para estimar suas distribuições desconhecidas.

Suponha, por exemplo, que $\omega_{0}$ seja o parâmetro de interesse. Para cada amostra, calculamos o EMV para $\omega_{0}$ e, ao final de $R$ reamostragens, temos $\hat{\omega}_{0_{1}}^{*}<\ldots<\hat{\omega}_{0_{R}}^{*}$ valores dos EMV ordenados. Utilizam-se então

$$
\hat{\omega}_{0_{1(R+1)(\alpha / 2)}^{*}}^{*} \text { e } \hat{\omega}_{0_{1(R+1)(1-\alpha / 2)}^{*}}^{*}
$$

como limites superior e inferior, respectivamente, do intervalo $100(1-\alpha) \%$ de confiança para $\omega_{0}$. Desta forma, através de (2.7) obtemos intervalos de confiança percentil "bootstrap" $100(1-\alpha) \%$ para o parâmetro de interesse. Intervalos de confiança percentil "bootstrap" para os outros parâmetros, $\beta_{0}$ e $\beta_{1}$, podem ser obtidos de maneira análoga.

Existem dois tipos básicos de métodos "bootstrap", o paramétrico e o não paramétrico. 
No "bootstrap" não paramétrico, os EMV's são obtidos através do modelo ajustado, isto é, geram-se dados do modelo ajustado com os valores dos parâmetros fixados nos EMV's obtidos da amostra original. No método paramétrico, os EMV's são baseados em $R$ reamostras com reposição obtidas da amostra original. Em geral, o número de amostragens $R$ é fixado em 999, como será considerado aqui. Maiores detalhes sobre os métodos "bootstrap" podem ser encontrados em (Efron e Tibishirami, 1993) e (Davison e Hinkley, 1997).

\subsubsection{Estimação intervalar via verossimilhança perfilada}

Uma outra possibilidade para fazer inferências sobre o vetor de parâmetros $\left(\beta_{0}, \beta_{1}, \omega_{0}\right)$ é usar verossimilhanças perfiladas (Kalbfleish, 1985). Suponha, por exemplo, que nosso interesse seja fazer inferências sobre o parâmetro $\omega_{0}$. A verossimilhança perfilada para tal parâmetro é dada por

$$
L_{p \text { erf }}\left(\omega_{0}\right)=\max _{\beta_{0}, \beta_{1}} L\left(\beta_{0}, \beta_{1}, \omega_{0}\right)
$$

e o intervalo de confiança $1-\alpha$ para $\omega_{0}$ é o conjunto tal que

$$
l_{p \text { erf }}\left(\omega_{0}\right)=\log L_{p \text { erf }}\left(\omega_{0}\right) \geq \log L_{p}\left(\widehat{\omega_{0}}\right)-\frac{1}{2} d_{1,1-\alpha}
$$

em que $d_{1,1-\alpha}$ é o $(1-\alpha)$-th quantil de uma distribuição $\chi_{1}^{2}$. Inferências para $\beta_{0}$ e $\beta_{1}$ são obtidas de maneira similar.

Os intervalos de confiança perfilados $100(1-\alpha) \%$ corrigidos via "bootstrap" paramétrico e não paramétrico para o parâmetro de interesse podem ser obtidos através da equação (2.9) substituindo $d_{1,1-\alpha}$ por $w_{1-\alpha}^{* 1}$ e $w_{1-\alpha}^{* 2}$, onde $w^{* 1}\left(\omega_{0}\right)=2\left\{l_{\text {perf }}^{* 1}\left(\hat{\omega}_{0}\right)-l_{p \text { erf }}^{* 1}\left(\omega_{0}\right)\right\}$ e $w^{* 2}\left(\omega_{0}\right)=2\left\{l_{p \text { erf }}^{* 2}\left(\hat{\omega}_{0}\right)-l_{p \text { erf }}^{* 2}\left(\omega_{0}\right)\right\}$ são as estatísticas da razão de verossimilhança baseadas em 999 reamostras com reposição da amostra original ("bootstrap"não paramétrico) e através do modelo ajustado original ("bootstrap" paramétrico), respectivamente.

\subsection{Análise Bayesiana}

Nesta seção, mostramos como uma abordagem Bayesiana pode ser utilizada como uma alternativa para os métodos frequentistas discutidos na seção anterior.

Supondo que uma distribuição a priori para $\psi=\left(\beta_{0}, \beta_{1}, \omega_{0}\right)$ seja dada por $\pi(\psi)=$ 
$\pi\left(\beta_{0}\right) \pi\left(\beta_{1}\right) \pi\left(\omega_{0}\right)$, e considerando a função de verosimilhança dada em (2.5), a densidade a posteriori conjunta (6.26) para $\psi$ pode ser escrita como:

$$
\begin{gathered}
\pi(\psi \mid \text { dados }) \alpha \pi\left(\beta_{0}\right) \pi\left(\beta_{1}\right) \pi\left(\omega_{0}\right) \prod_{i=1}^{m} \prod_{j=1}^{n_{i}}\left\{\left\{\exp \left(-\beta_{0}+\beta_{1} \log \left(x_{i}-\omega_{0}\right)\right)\right\}^{\delta_{i j}}\right. \\
\left.\left\{\exp \left[-\exp \left(-\beta_{0}+\beta_{1} \log \left(x_{i}-\omega_{0}\right)\right)\right] t_{i j}\right\}\right\} .
\end{gathered}
$$

Inferências sobre os parâmetros podem ser baseadas em suas densidades a posteriori marginais, as quais são obtidas integrando (2.10) com relação a cada um dos parâmetros $\left(\beta_{0}, \beta_{1}, \omega_{0}\right)$. Desde que não é possível obter as distribuições a posteriori marginais para cada parâmetro explicitamente, algum método de aproximação de integrais deve ser empregado. Por exemplo, pode-se utilizar um dos métodos de simulação baseados em cadeias de Markov (Gibbs-Sampling, Metropolis-Hastings) ou métodos de aceitaçãorejeição (ARS, ARMS, etc), descritos no Apêndice B, assim como métodos numéricos para aproximar as integrais de interesse (método de Laplace, método da quadratura Gaussiana, etc).

\subsubsection{O método MCMC}

Como descrito no Apêndice B, para tratar o problema de estimação Bayesiana via métodos de simulação é importante explicitar da maneira mais conveniente possível as posteriores condicionais de cada parâmetro, de tal modo que as observações possam ser geradas a partir das mesmas. Caso essas posterioris condicionais pertençam a alguma família de distribuições conhecida- fato incomum na maioria das aplicações- o procedimento de estimação é facilmente viabilizado por meio do algoritmo Gibbs-Sampling (veja, por exemplo, Gelfand e Smith, 1990).

Para evitar instabilidade numérica, consideramos a reparametrização $\omega_{0}=x_{1}-\exp (\rho)$, proposta por Davison e Hinkley (1997), e distribuições a priori da forma $\pi(\psi) \backsim$ $N\left(\mu_{i}, \sigma_{i}^{2}\right)$, com $\mu_{i}=0$, para cada um dos parâmetros $\beta_{0}, \beta_{1}$ e $\rho$, as posterioris condicionais são dadas, respectivamente, por

$$
\pi\left(\beta_{0} \mid \beta_{1}, \rho ; x\right) \alpha \exp \left\{\sum_{i=1}^{m} \sum_{j=1}^{n_{i}}-\delta_{i j} \beta_{0}-t_{i j} \exp \left(-\beta_{0}+\beta_{1} \log \left(x_{i}-x_{1}+\exp (\rho)\right)\right)-\frac{\beta_{0}^{2}}{2 \sigma_{1}^{2}}\right\}
$$




$$
\begin{gathered}
\pi\left(\beta_{1} \mid \beta_{0}, \rho ; x\right) \alpha \exp \left\{\sum_{i=1}^{m} \sum_{j=1}^{n_{i}} \delta_{i j} \beta_{1} \log \left(x_{i}-x_{1}+\exp (\rho)\right)-\right. \\
\left.t_{i j} \exp \left(-\beta_{0}+\beta_{1} \log \left(x_{i}-x_{1}+\exp (\rho)\right)\right)-\frac{\beta_{1}^{2}}{2 \sigma_{2}^{2}}\right\} \\
\pi\left(\rho \mid \beta_{0}, \beta_{1} ; x\right) \alpha \exp \left\{\sum_{i=1}^{m} \sum_{j=1}^{n_{i}} \delta_{i j} \beta_{1} \log \left(x_{i}-x_{1}+\exp (\rho)\right)-\right. \\
\left.t_{i j} \exp \left(-\beta_{0}+\beta_{1} \log \left(x_{i}-x_{1}+\exp (\rho)\right)\right)-\frac{\rho^{2}}{2 \sigma_{3}^{2}}\right\}
\end{gathered}
$$

Dadas as posterioris condicionais de $\beta_{0}, \beta_{1}$ e $\omega_{0}$, podemos observar que elas não pertencem a nenhuma família paramétrica conhecida de distribuições. Assim, amostrar valores diretamente das mesmas é, em princípio, impossível. Utilizamos então o algoritmo de rejeição adaptativo para densidades log-côncavas ARS (Gilks, 1992; Gilks e Wild, 1992), descrito no Apêndice B. Para a sua utilização, devemos verificar a log-concavidade das posterioris condicionais (2.11), (2.12) e (2.13). Mostraremos aqui apenas a log-concavidade da posteriori condicional de $\beta_{0}$, uma vez que, para os outros parâmetros, a verificação é feita de forma similar. Temos que

$$
\begin{aligned}
\frac{\partial \pi\left(\beta_{0} \mid \beta_{1}, \rho ; x\right)}{\partial \beta_{0}}= & \exp \left\{\sum_{i=1}^{m} \sum_{j=1}^{n_{i}}-\delta_{i j} \beta_{0}-t_{i j} \exp \left(-\beta_{0}+\beta_{1} \log \left(x_{i}-x_{1}+\exp (\rho)\right)\right)-\frac{\beta_{0}^{2}}{2 \sigma_{1}^{2}}\right\} \\
& \left(\sum_{i=1}^{m} \sum_{j=1}^{n_{i}}\left[-\delta_{i j}+t_{i j} \exp \left(-\beta_{0}+\beta_{1} \log \left(x_{i}-x_{1}+\exp (\rho)\right)\right)\right]-\frac{\beta_{0}}{\sigma_{1}^{2}}\right),
\end{aligned}
$$

e portanto

$$
\frac{\partial^{2} \pi\left(\beta_{0} \mid \beta_{1}, \rho ; x\right)}{\partial \beta_{0}^{2}}=\exp \{B\}\left(\frac{\partial^{2} B}{\partial \beta_{0}^{2}}-\left(\frac{\partial B}{\partial \beta_{0}}\right)^{2}\right)
$$

onde $B=\left\{\sum_{i=1}^{m} \sum_{j=1}^{n_{i}}-\delta_{i j} \beta_{0}-t_{i j} \exp \left(-\beta_{0}+\beta_{1} \log \left(x_{i}-x_{1}+\exp (\rho)\right)\right)-\frac{\beta_{0}^{2}}{2 \sigma_{1}^{2}}\right\}$. A log-concavidade da posteriori condicional de $\beta_{0}$ decorre então de (2.14) e do fato de que

$$
\frac{\partial^{2} B}{\partial \beta_{0}^{2}}=\sum_{i=1}^{m} \sum_{j=1}^{n_{i}}-t_{i j} \exp \left(-\beta_{0}+\beta_{1} \log \left(x_{i}-x_{1}+\exp (\rho)\right)\right)-\frac{1}{\sigma_{1}^{2}}<0
$$




\subsubsection{O método de Laplace}

Um dos métodos mais utilizados em inferência Bayesiana aproximada é o método de Laplace para aproximação de integrais (Kass, Tierney e Kadane, 1990). Os métodos analíticos de aproximação são mais eficientes computacionalmente, porém são baseados em resultados assintóticos e na suposição de normalidade, o que é um problema quando a amostra é pequena. Também são mais difíceis de ser implementados, em termos de programação, à medida que o número de parâmetros aumenta.

Suponha que nosso interesse seja em $\omega_{0}$. Utilizando uma priori não informativa de Jeffreys para os parâmetros, dada por: $\pi\left(\beta_{0}, \beta_{1}, \omega_{0}\right) \alpha\left\{\operatorname{det} I\left(\beta_{0}, \beta_{1}, \omega_{0}\right)\right\}^{1 / 2}$, onde $I$ (.) é a matriz de informação de Fischer, obtemos pelo método de Laplace a densidade a posteriori marginal aproximada para $\omega_{0}$, dada por

$$
\pi\left(\omega_{0} \mid \text { dados }\right) \alpha(2 \pi)^{d / 2}\left|D_{-r}^{*}\right|^{-1 / 2} \pi\left(\zeta^{*} \mid \text { dados }\right)
$$

em que $d$ é a dimensão de $\zeta, \zeta^{*}=\left(\hat{\beta}_{0}, \hat{\beta}_{1}, \omega_{0}\right)$ são os EMV's de $\left(\beta_{0}, \beta_{1}\right)$, com $\omega_{0}$ fixo, e $D_{-r}^{*}$ é o determinante da matriz Hessiana a menos da densidade a posteriori conjunta para $\beta_{0}$ e $\beta_{1}$ calculada em $\zeta^{*}$. Inferências para $\omega_{0}$ podem então ser obtidas a partir da densidade a posteriori marginal (2.15). De modo análogo podemos obter inferências para $\beta_{0}$ e $\beta_{1}$.

\subsection{Aplicações}

\subsubsection{Dados simulados}

O estudo de simulação foi baseado em amostras geradas de uma distribuição exponencial. Usando a relação estresse resposta geral com o modelo de lei da potência inversa (1.12), os valores dos parâmetros foram fixados em $\beta_{0}=2.0, \beta_{1}=2.0$ e $\omega_{0}=4.8$. Utilizamos três níveis de estresse $x=5,10,15$, com 20 observações não censuradas em cada nível. Esse conjunto de dados já foi estudado na Seção 1.8, onde vimos através de técnicas gráficas que ele é bem ajustado pelo modelo exponencial. 


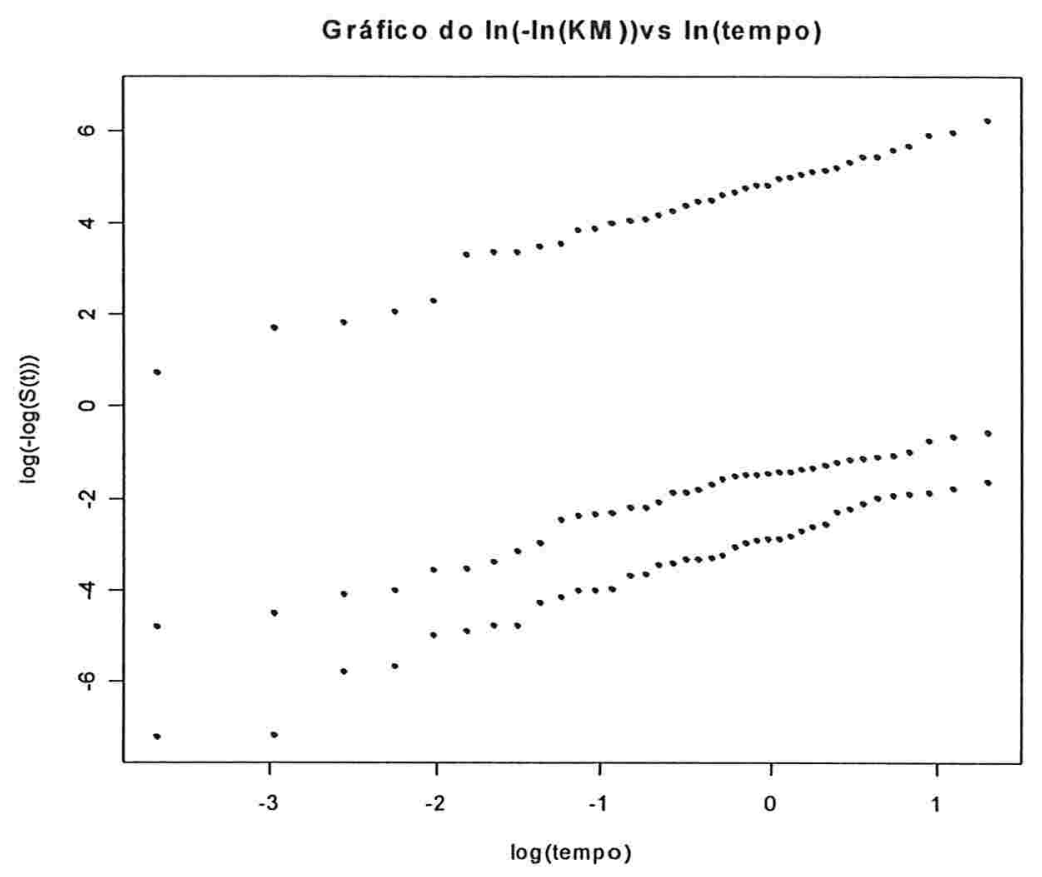

Figura 2.2: Gráfico de $\log (-\log (S(t)))$ por $\log (t)$.

Dados simulados com $\beta_{0}=2.0, \beta_{1}=2.0$ e $\omega_{0}=4.8$ e $n=20$.

A Tabela 2.1 apresenta as estimativas de máxima verossimilhança e seus intervalos de confiança de $95 \%$ calculados via teoria assintótica, verossimilhança perfilada e corrigida via "bootstrap" paramétrico e não paramétrico, e através de métodos "bootstrap" paramétrico e não paramétrico. Também estão apresentadas as estimativas Bayesianas calculadas via método MCMC e Laplace e seus respectivos intervalos de credibilidade de 95\% para os parâmetros do modelo, com os respectivos erros quadráticos médios. As estimativas de máxima verossimilhança foram obtidas usando a rotina $n l m$ do pacote $R$, a qual determina um mínimo local de uma função não linear usando o método geral de quasi-Newton. Os procedimentos para a obtenção dos intervalos de confiança clássicos, via "bootstrap" paramétrico e não paramétrico, foram implementados no pacote SPLUS. Para a análise Bayesiana, consideramos densidades a priori vagas normais com $\mu=0 \mathrm{e}$ $\sigma^{2}=10^{4}$ para os parâmetros $\beta_{0}, \beta_{1}, \rho$. O método de Laplace foi implementado também no pacote $S P L U S$, enquanto que o método de rejeição adaptativo (ARS) no software BUGS (Spiegelhalter et al., 1995).

Para monitorar os comportamentos das cadeias, o software CODA foi utilizado. Foram consideradas 3 cadeias de tamanho 34000 cada. As 4000 primeiras iterações de cada cadeia foram descartadas. Para cada cadeia, foram tomados valores de 11 em 11, obtendo-se as- 
sim uma cadeia de tamanho 8184. Este critério de seleção foi sugerido pelo diagnóstico de convergência de Raftery e Lewis (1992). Através do diagnóstico de convergência de Gelman-Rubin, obtivemos valores de $\hat{\mathrm{R}}=1.002 ; 1.002 ; 1.001$ para $\beta_{0}$, $\beta_{1}$ e $\rho$, respectivamente. Tais valores trouxeram fortes indícios de convergência para a densidade a posteriori dos parâmetros. Os outros critérios também indicaram convergência.

Podemos observar que os intervalos de confiança calculados através da teoria assintótica foram muito maiores que os intervalos calculados via métodos Bayesianos, com exceção dos métodos de verossimilhança perfilada corrigida via bootstrap paramétrico e não paramétrico e os métodos de bootstrap. Tais resultados podem ser explicados pelo fato conhecido de que em amostras pequenas a teoria assintótica não funciona muito bem.

Uma análise de sensibilidade de prioris foi realizada com diferentes valores dos hiperparâmetros, isto é, consideramos prioris $N\left(0, \sigma_{i}^{2}\right)$ com $\sigma_{i}^{2}=10^{2}, 10^{3}, 10^{4}$ para $i=1, \ldots, 3$. Os resultados obtidos com esses valores foram muito similares.

Na Figura 2.3, são apresentados os gráficos das posterioris marginais e a história das iterações do algoritmo ARs, na Figura 2.4, as densidades a posteriori de Laplace e, na Figura 2.5 as autocorrelações com lag 1 e 11 respectivamente. 
Tabela 2.1: Estimativas clássica e Bayesiana e IC de $95 \%$ para os parâmetros

\begin{tabular}{|c|c|c|c|}
\hline Procedimentos & $\hat{\beta}_{0}$ & $\hat{\beta}_{1}$ & $\hat{\omega}_{0}$ \\
\hline EMV & 1.36 & 2.06 & 4.770 \\
& $\begin{array}{c}(E Q M=0.52) \\
(-1.28 ; 4.32)\end{array}$ & $\begin{array}{c}(E Q M=0.36) \\
(0.43 ; 3.78)\end{array}$ & $\begin{array}{c}(E Q M=0.0009) \\
(2.42 ; 4.988)\end{array}$ \\
\hline V. Perfilada & $(-0.56 ; 2.85)$ & $(0.38 ; 3.33)$ & $(4.57 ; 4.99)$ \\
\hline V. Perf. (boot. paramétrico) & $(1.01 ; 2.33)$ & $(1.51 ; 2.44)$ & $(4.79 ; 4.95)$ \\
\hline V. Perf. (boot. não paramét.) & $(1.02 ; 2.39)$ & $(1.49 ; 2.41)$ & $(4.70 ; 4.86)$ \\
\hline Bootstrap paramétrico & $(0.99 ; 2.44)$ & $(1.48 ; 2.47)$ & $(4.64 ; 4.97)$ \\
\hline Bootstrap não-paramétrico & $(1.06 ; 2.28)$ & $(1.61 ; 2.32)$ & $(4.68 ; 4.89)$ \\
\hline MCMC & 1.748 & 1.874 & 4.823 \\
& $(E Q M=0.06)$ & $(E Q M=0.01)$ & $(E Q M=0.0006)$ \\
& $(0.77 ; 3.26)$ & $(0.45 ; 2.55)$ & $(4.36 ; 4.998)$ \\
\hline Laplace & 1.790 & 1.899 & 4.781 \\
& $(E Q M=0.04)$ & $(E Q M=0.01)$ & $(E Q M=0.0004)$ \\
& $(0.78,2.89)$ & $(1.32 ; 2.59)$ & $(4.45 ; 4.99)$ \\
\hline
\end{tabular}

Dados simulados com $\beta_{0}=2.0, \beta_{1}=2.0$ e $\omega_{0}=4.8$ e $n=20$. 

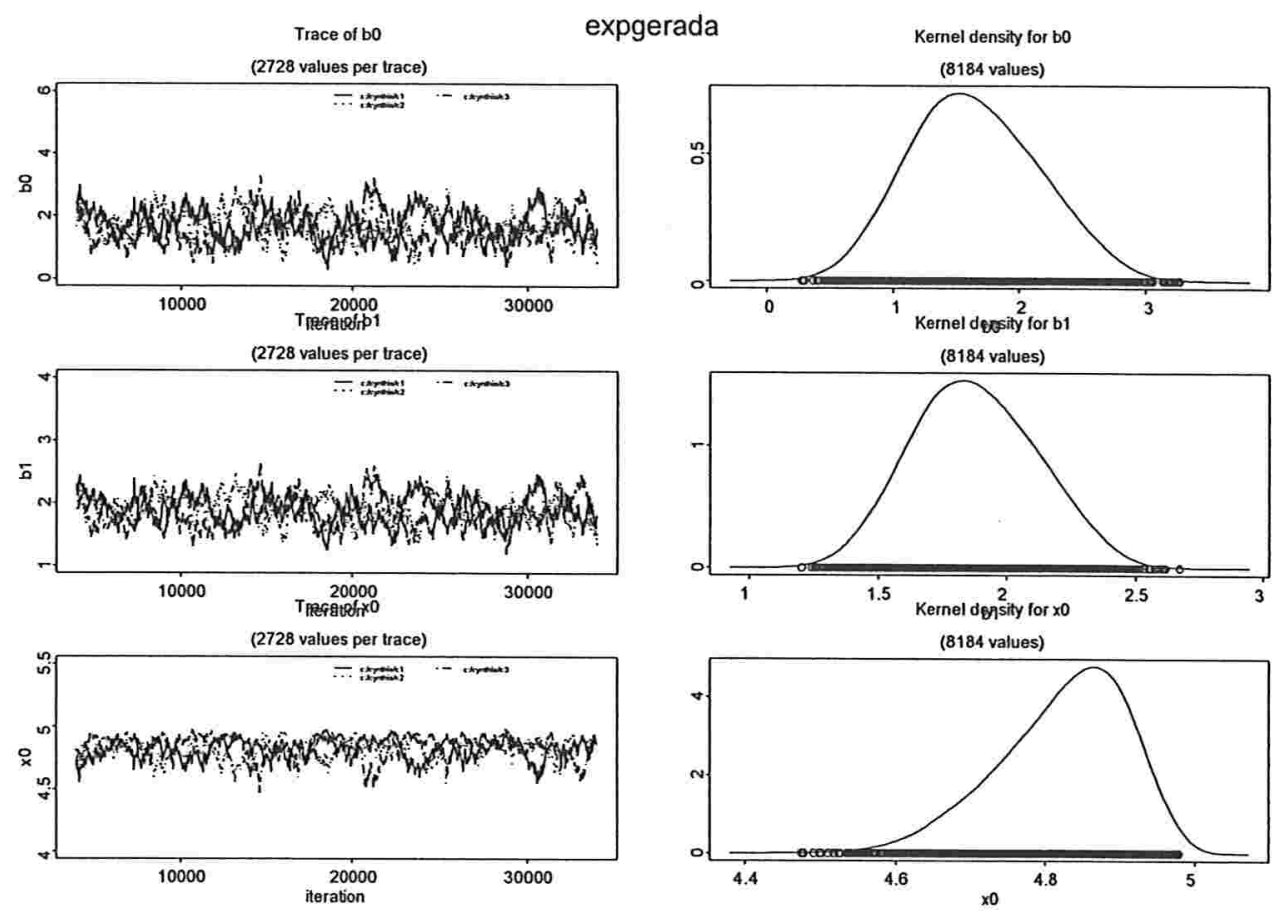

Figura 2.3 : Gráficos das posterioris marginais e a história das iterações do algoritmo ARS 

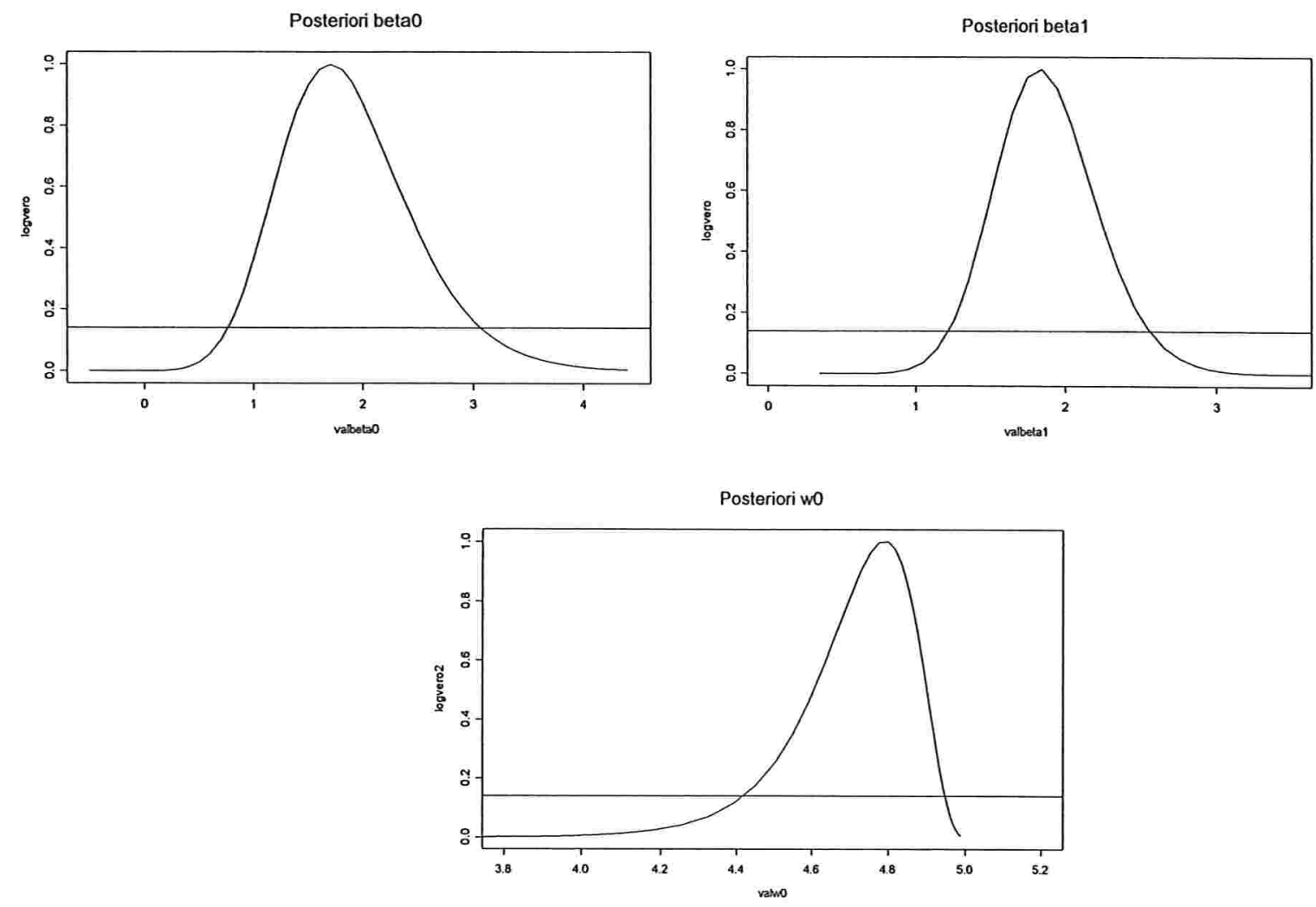

Figura 2.4: Densidades a posteriori de Laplace. 

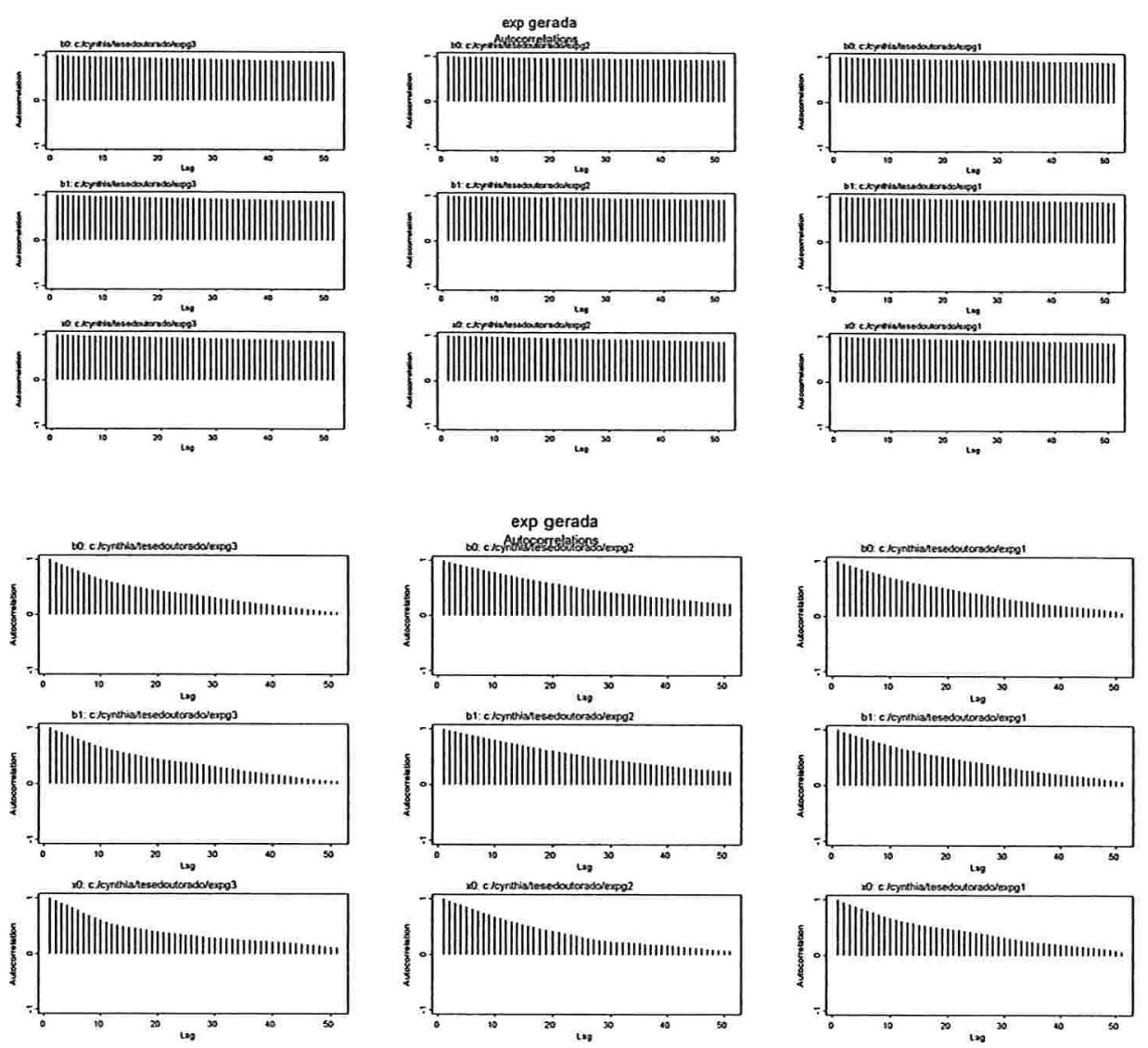

Figura 2.5: Autocorrelações para lag=1 e lag=11 respectivamente.

\subsubsection{Dados reais}

Na Tabela 2.2 são apresentados os dados referentes a tempos de vida (em horas) de uma película usada como isolante elétrico Hirose (1993). Os tempos até a falha foram observados em 4 níveis diferentes de estressse $x=5,7,10$ e 15 quilovolts-ampéres com, respectivamente, 10, 15, 10 e 9 observações em cada nível. Foram observados três valores censurados em $x=5$. Embora os métodos gráficos descritos na seção 1.8 tenham indicado que esse conjunto de dados seria melhor ajustado por uma distribuição Weibull com parâmetro de forma dependente dos níveis de estresse, ele será utilizado nesta seção apenas a título de ilustração. 
Tabela 2.2: tempos de resistência de películas isolantes

\begin{tabular}{|l|l|l|}
\hline Voltagem $\left(x_{i}\right)$ & $N$ & \multicolumn{1}{|c|}{ tempos até a falha (horas) } \\
\hline 5 & 10 & $7131,8482,8559,8762,9026,9034,9104,+,+,+$ \\
\hline 7 & 15 & $\begin{array}{l}50.25,87.75,87.76,87.77,92.90,92.91,95.96,108.30, \\
108.30,117.90,123.90,124.30,129.70,135.60,135.60\end{array}$ \\
\hline 10 & 10 & $\begin{array}{l}15.17,19.87,20.18,21.50,21.88,22.23,23.02,23.90, \\
28.17,29.70\end{array}$ \\
\hline 15 & 9 & $2.40,2.42,3.17,3.75,4.65,4.95,6.23,6.68,7.30$ \\
\hline
\end{tabular}

+indica tempos censurados.

A Tabela 2.3 apresenta as estimativas de máxima verossimilhança (médias) e seus intervalos de confiança de $95 \%$ calculados via teoria assintótica, verossimilhança perfilada e corrigida via "bootstrap" paramétrico e não paramétrico, e através de métodos "bootstrap" paramétrico e não paramétrico. Também estão apresentadas as estimativas Bayesianas (médias a posteriori) calculadas via método MCMC e Laplace e seus respectivos intervalos de credibilidade de $95 \%$ para os parâmetros do modelo. Como no caso de dados gerados, as estimativas de máxima verossimilhança foram obtidas usando a rotina $n l m$ do pacote $R$. Os procedimentos para obtenção dos intervalos de confiança clássicos, via "bootstrap" paramétrico e não paramétrico, foram implementados no pacote SPLUS. Para a análise Bayesiana, consideramos densidades a priori vagas normais com $\mu=0 \mathrm{e}$ $\sigma^{2}=10^{4}$ para os parâmetros $\beta_{0}, \beta_{1}, \rho$. O método de Laplace foi implementado também no pacote $S P L U S$, enquanto que o método de rejeição adaptativo (ARS) no software BUGS (Spiegelhalter et al., 1995).

Para monitorar os comportamentos das cadeias, o software CODA foi utilizado. Foram consideradas 3 cadeias de tamanho 34000 cada. As 4000 primeiras iterações de cada cadeia foram descartadas. Para cada cadeia, foram tomados valores de 7 em 7, obtendo-se assim uma cadeia de tamanho 12857. Esse critério de seleção foi sugerido pelo diagnóstico de convergência de Raftery e Lewis (1992). Através do diagnóstico de convergência de Gelman-Rubin, obtivemos valores de $\hat{\mathrm{R}}=1.002 ; 1.003 ; 1.0001$ para $\beta_{0}, \beta_{1}$ e $\rho$, respectivamente. Tais valores trazem fortes indícios de convergência para a densidade a posteriori dos parâmetros. Os critérios de Geweke (1992) e Heidelberger e Welch (1983) também 
indicaram convergência.

Novamente uma análise de sensibilidade de prioris foi realizada com diferentes valores dos hiperparâmetros, isto é, consideramos prioris $N\left(0, \sigma_{i}^{2}\right)$ com $\sigma_{i}^{2}=10^{2}, 10^{3}, 10^{4}$ para $i=1, \ldots, 3$. Os resultados obtidos com esses valores foram muito similares. Assim, apresentamos aqui somente os resultados para $N\left(0, \sigma_{i}^{2}\right)$ com $\sigma_{i}^{2}=10^{4}$.

A Figura 2.6 mostra o tempo médio de vida $\theta_{i}=1 / \lambda_{i}=g\left(\beta_{0}, \beta_{1}, \omega_{0}\right)$ para um nível de estresse $i$ como uma função da voltagem, com o parâmetro de estresse limiar estimado através do método MCMC.

Na Figura 2.7, são apresentados os gráficos das posterioris marginais e a história das iterações do algoritmo ARS, e na Figura 2.8 as densidades a posteriori de Laplace.

Tabela 2.3: Estimativas clássica e Bayesiana e IC de $95 \%$ para os parâmetros

\begin{tabular}{|c|c|c|c|}
\hline Procedimentos & $\hat{\beta}_{0}$ & $\hat{\beta}_{1}$ & $\hat{\omega}_{0}$ \\
\hline EMV & 6.303 & 2.015 & 4.787 \\
& $(6.22 ; 6.88)$ & $(1.96 ; 2.06)$ & $(4.70 ; 4.80)$ \\
\hline V. Perfilada & $(5.47 ; 7.48)$ & $(1.51 ; 2.62)$ & $(4.49 ; 4.93)$ \\
\hline V. Perf. (boot. paramétrico) & $(5.75 ; 6.98)$ & $(1.48 ; 2.52)$ & $(4.58 ; 4.92)$ \\
\hline V. Perf. (boot. não paramét.) & $(5.76 ; 6.89)$ & $(1.44 ; 2.49)$ & $(4.60 ; 4.86)$ \\
\hline Bootstrap paramétrico & $(5.73 ; 7.02)$ & $(1.47 ; 2.57)$ & $(4.54 ; 4.97)$ \\
\hline Bootstrap não-paramétrico & $(6.02 ; 6.63)$ & $(1.84 ; 2.23)$ & $(4.69 ; 4.87)$ \\
\hline MCMC & 6.353 & 2.012 & 4.69 \\
& $(5.28 ; 7.75)$ & $(1.34 ; 2.75)$ & $(4.32 ; 4.96)$ \\
\hline Laplace & 6.30 & 2.00 & 4.78 \\
& $(5.25,7.56)$ & $(1.35 ; 2.70)$ & $(4.38 ; 4.94)$ \\
\hline
\end{tabular}




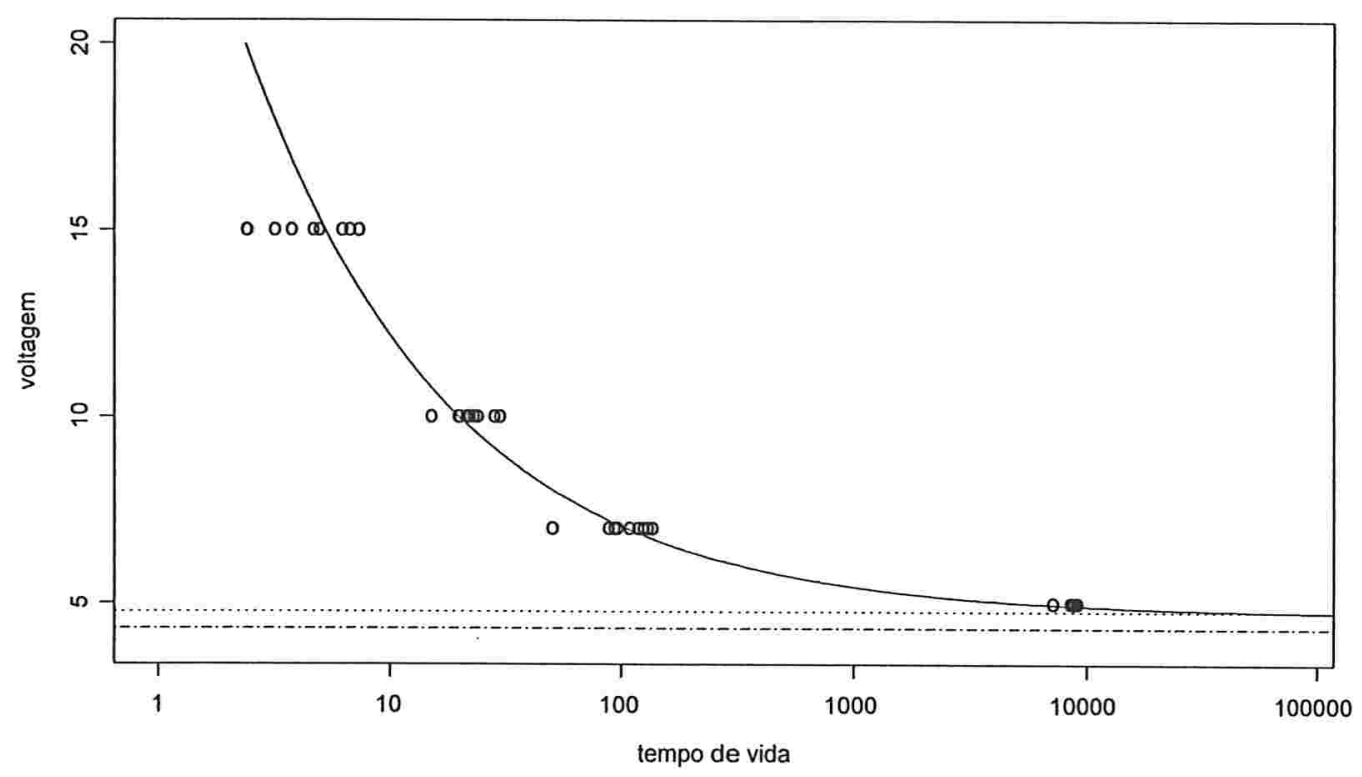

Figura 2.6: Gráfico do tempo médio ajustado como uma função da voltagem (-) com a voltagem "limiar"estimada (- - -) e intervalo de credibilidade de $95 \%$ obtido via Laplace
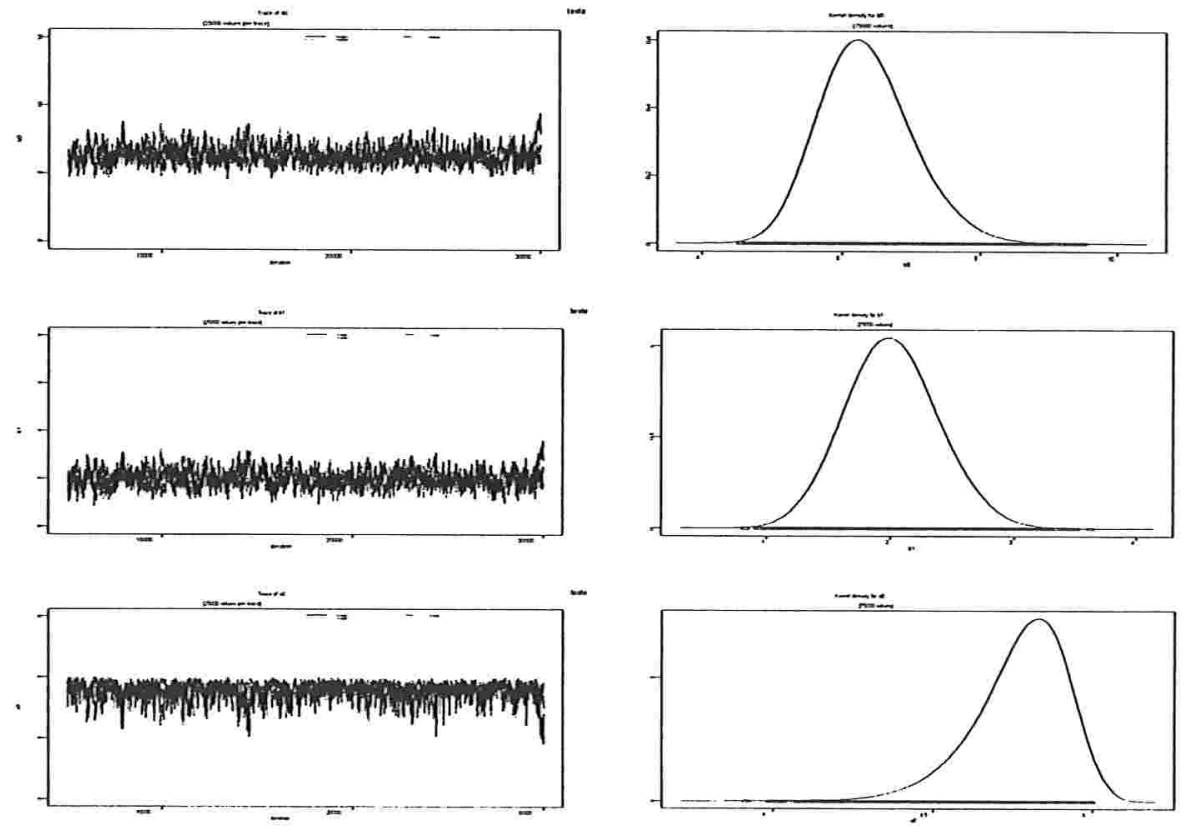

Figura 2.7 : Gráficos das posterioris marginais e a história das iterações do algoritmo ARS 

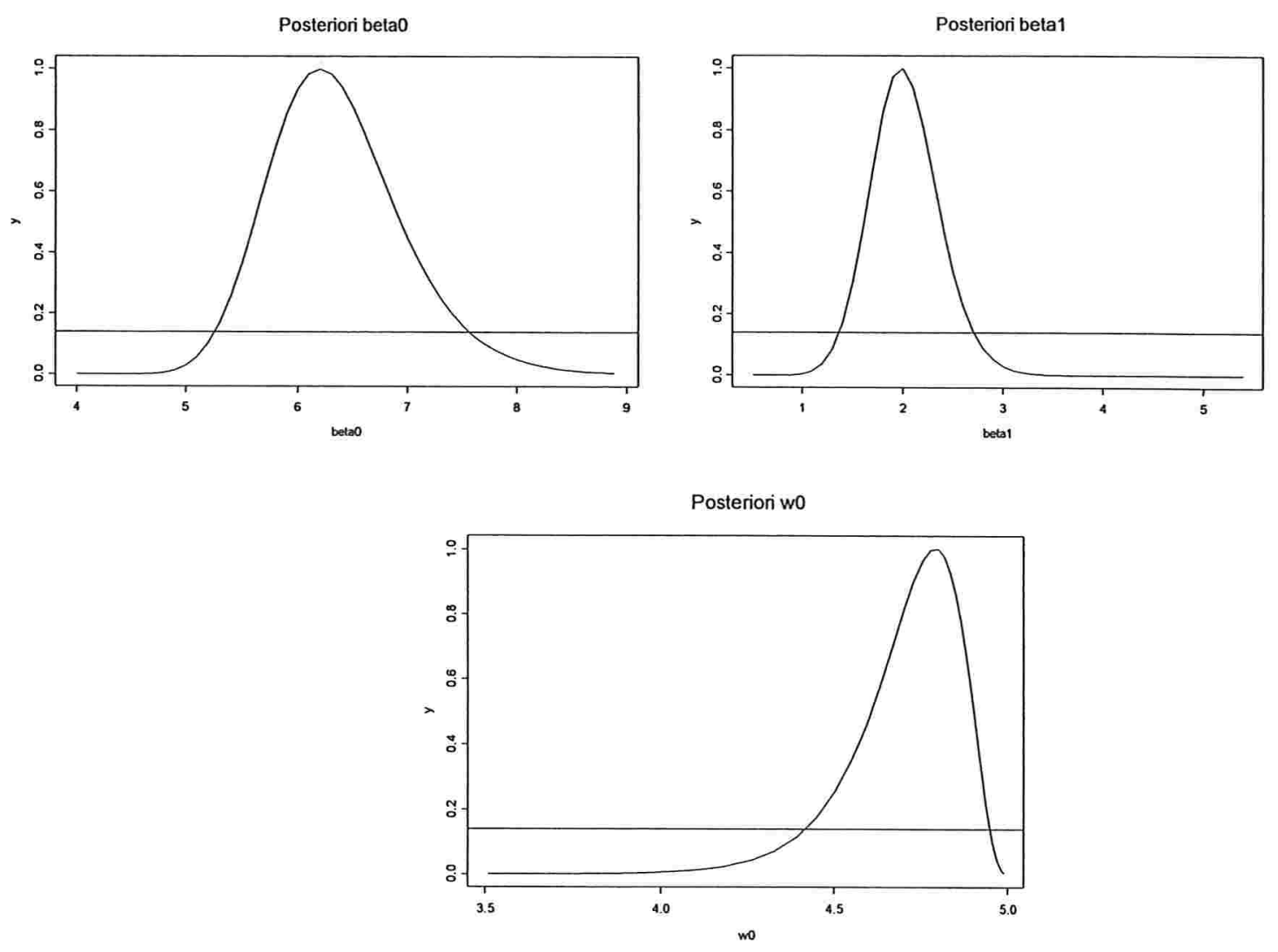

Figura 2.8: Densidades a posteriori de Laplace.

\subsection{Conclusões}

Neste capítulo, apresentamos metodologias para a modelagem de testes de sobrevivência acelerados sob um modelo de lei da potência inversa com um estresse limiar, cuja estimação foi nosso principal interesse. Propusemos uma relação estresse-resposta bastante geral em (1.10), da qual as relações estresse- resposta mais conhecidas em análise de sobrevivência e confiabilidade são casos particulares. No entanto, como no restante do trabalho, utilizamos como relação estresse-resposta somente o modelo de lei da potência inversa com estresse limiar, embora a metodologia proposta seja geral e possa ser aplicada para outras relações estresse-resposta. De maneira geral, os objetivos de apresentar situações em que existe o estresse limiar e de como estimá-lo foi atingido.

Nossa metodologia foi apresentada em termos da função de risco. A utilização da distribuição exponencial para expressar o comportamento dos tempos de vida justifica-se por sua simplicidade e adequação a situações em que a função de risco é constante.

Apresentamos um exemplo baseado em dados gerados em que pareceu ser razoável 
admitirr que a forma da distribuição dos tempos de vida fosse independente dos níveis de estresse. Para isso utilizamos técnicas gráficas, como os gráficos do risco empírico acumulado. Finalmente, como ilustração aplicamos a metodologia a um conjunto de dados reais.

Para a estimação dos parâmetros, utilizamos análises clássicas baseadas na normalidade assintótica dos parâmetros envolvidos, e métodos de simulação "bootstrap" paramétrico e não paramétrico, além do método de verossimilhança parcial. Sob o enfoque Bayesiano, utilizamos técnicas de MCMC e transformadas de Laplace. As amostras para os parâmetros de interesse foram simuladas via "Bugs" ou programadas em linguagem $\mathrm{S}$ e R. Em todos os casos obtivemos as estimativas (médias e médias a posteriori) dos parâmetros de interesse e não tivemos problemas de convergência. Comparamos as estimativas Bayesianas com aquelas obtidas via análise clássica, em um conjunto de dados reais e constatamos que os resultados foram muito próximos para todos os parâmetros, o que não ocorreu para um conjunto de dados artificiais com $n=20$. 


\section{Capítulo 3}

\section{Modelos de Riscos Híbridos}

\section{PH/AFT com Estresse Limiar}

Neste capítulo, propomos um modelo de riscos híbrido que, além de conter os modelos de riscos proporcionais de Cox (PH) e o modelo de sobrevivência acelerado (AFT) como casos particulares, inclui ainda um estresse limiar. P.ara a representação da função de risco base no modelo, adotamos uma abordagem paramétrica baseada na distribuição Weibull, generalizando assim o modelo do capítulo anterior.

Para a estimação dos parâmetros, consideramos as abordagens clássica e Bayesiana. Através de um estudo de simulação, mostramos as probabilidades de cobertura dos intervalos de confiança para os parâmetros através da teoria assintótica usual. A metodologia é ilustrada com um conjunto de dados reais de modelos AFT.

\subsection{O modelo de riscos híbrido com estresse limiar}

O modelo de riscos híbrido com estresse limiar proposto neste capítulo é dado por

$$
h(t \mid z)=g_{1}\left(\alpha_{1}^{T}\left(z-\omega_{0}\right)\right) h_{0}\left(g_{2}\left(\alpha_{2}^{T}\left(z-\omega_{0}\right)\right) t ; \phi\right),
$$

onde $g_{1}(),. g_{2}($.$) são funções monótonas positivas conhecidas que assumem o valor 1$ quando seus argumentos são zero, isto é, $g_{1}(0)=1=g_{2}(0), z$ é um vetor de covariáveis, $\alpha_{1}, \alpha_{2}$ e $\phi$ são vetores de parâmetros de regressão desconhecidos, $\omega_{0}$ é o vetor de parâmetros desconhecidos de estresses limiares, com $z_{i}>\omega_{0_{i}}$ para todo $i$, e $h_{0}($.$) é a função de$ risco base. Uma grande variedade de formas pode ser empregada para $g_{1}($.$) e g_{2}($.$) , mas$ a candidata mais natural para ambas é a função exponencial. 
Uma vantagem desta formulação é admitir várias classes de modelos de riscos como casos particulares. Quando $\alpha_{1}=\alpha_{2}$ temos o modelo de falha acelerada (AFT) (Prentice, 1978) e, para $\alpha_{2}=0$, o modelo de riscos proporcionais de Cox (PH) (Cox, 1972), mas agora com o estresse limiar. Consequentemente, ambos os modelos PH e AFT são casos particulares de (3.1). O modelo (3.1) reduz-se ao modelo usual de riscos híbrido quando $\omega_{0}=0$ (Etezadi-Amoli and Ciampi, 1987).

O modelo (3.1) é semi-paramétrico se a forma da função de risco base $h_{0}($.$) é deter-$ minada não parametricamente a partir dos dados. Para um modelo híbrido PH/AFT, Ciampi e Etezadi-Amoli (1985) consideram uma aproximação polinomial para a função de risco base, enquanto que Etezadi-Amoli e Ciampi (1987) utilizam uma aproximação "spline". Tais abordagens não serão consideradas nesta tese. Do ponto de vista paramétrico, supondo $g_{1}()=.g_{2}()=.g(.) \mathrm{e}$

$$
h_{0}(u ; \phi)=\phi u^{\phi-1}
$$

em que $u=\lambda t$ e $\lambda$ é definida como em (1.12), isto é, $\lambda=\exp \left(-\beta_{0}+\beta_{1} \log \left(x-\omega_{0}\right)\right)$, o modelo (3.1) corresponde à função de risco de uma variável aleatória com uma distribuição Weibull com 2 parâmetros (Lawless, 1982), um dos quais, $\lambda=g\left(\alpha^{T}\left(z-\omega_{0}\right)\right)$, dependendo de covariáveis, e do parâmetro de estresse limiar, $\omega_{0}$. Quando $\phi=1$, obtemos a partir de (3.1) a função de risco da distribuição exponencial. Para o caso exponencial, temos que (3.1) fica então dado por

$$
h(t \mid z)=\lambda=g\left(\alpha^{T}\left(z-\omega_{0}\right)\right)=\exp \left(\alpha^{T}\left(z-\omega_{0}\right)\right)
$$


Tabela 3.1: Relações entre os modelos de riscos supondo $g_{1}=g_{2}=g$. A função de risco base é denotada por $h_{0}$ e o parâmetro de estresse limiar por $\omega_{0}$.

\begin{tabular}{lcc}
\hline \hline Modelo & Restrição & Risco \\
\hline PH/AFT com " $\omega_{0} "$ & - & $g\left(\alpha_{1}^{T}\left(z-\omega_{0}\right)\right) h_{0}\left(g\left(\alpha_{2}^{T}\left(z-\omega_{0}\right)\right) t ; \phi\right)$ \\
AFT com " $\omega_{0} "$ & $\alpha_{1}=\alpha_{2}=\alpha$ & $g\left(\alpha^{T}\left(z-\omega_{0}\right)\right) h_{0}\left(g\left(\alpha^{T}\left(z-\omega_{0}\right)\right) t ; \phi\right)$ \\
PH com " $\omega_{0} "$ & $\alpha_{2}=0$ & $g\left(\alpha_{1}^{T}\left(z-\omega_{0}\right)\right) h_{0}(t ; \phi)$ \\
PH/AFT & $\omega_{0}=0$ & $g\left(\alpha_{1}^{T} z\right) h_{0}\left(g\left(\alpha_{2}^{T} z\right) t ; \phi\right)$ \\
\hline \hline AFT & $\alpha_{1}=\alpha_{2}=\alpha$ e $\omega_{0}=0$ & $g\left(\alpha^{T} z\right) h_{0}\left(g\left(\alpha^{T} z\right) t ; \phi\right)$ \\
\hline \hline PH & $\alpha_{2}=0$ e $\omega_{0}=0$ & $g\left(\alpha_{1}^{T} z\right) h_{0}(t ; \phi)$ \\
\hline \hline
\end{tabular}

\section{$3.2 \mathrm{O}$ modelo Weibull}

A distribuição Weibull foi proposta originalmente por Weibull (1951) e, desde então, tem sido amplamente utilizada em aplicações biométricas e industriais, por exemplo, em estudos sobre o tempo de ocorrência de tumores em populações humanas (Whittemore e Altschuler, 1976) ou sobre o tempo de vida de equipamentos industriais, dentre muitas outras situações. Sua função de densidade de probabilidade, função de sobrevivência e função de risco são dadas, respectivamente, por

$$
f(t)=\phi \lambda(\lambda t)^{\phi-1} \exp \left[-(\lambda t)^{\phi}\right], \quad S(t)=\exp \left[-(\lambda t)^{\phi}\right]
$$

e

$$
h(t)=\phi \lambda^{\phi} t^{\phi-1}, \quad t \geq 0, \quad \phi \text { e } \lambda>0 .
$$

Uma das características importantes da distribuição Weibull na modelagem de tempos de sobrevivência está relacionada à sua flexibilidade em acomodar diferentes formas da função de risco. Diferentemente da distribuição exponencial, cuja função de risco é constante, na distribuição Weibull temos funções de risco monótonas decrescentes para valores $\phi<1$ do parâmetro de forma, funções de risco monótonas crescentes quando $\phi>1$ e, para $\phi=1$, reobtemos a distribuição exponencial com função de risco constante (veja Figura abaixo). 

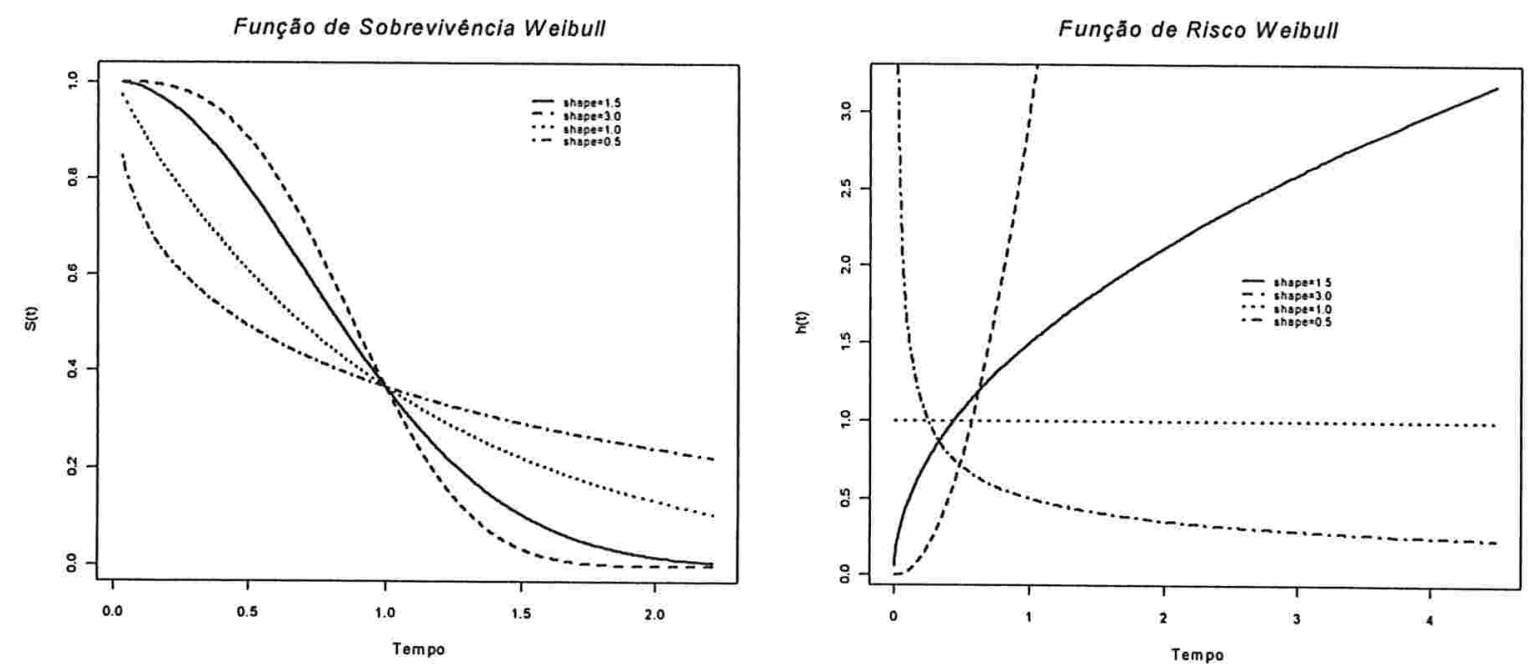

Figura 3.1: Gráfico das funções de sobrevivência e risco da distribuição Weibull para diferentes valores de $\phi$.

Considerando $h_{0}$ como em (3.2), supondo $g_{1}()=.g_{2}($.$) no modelo (3.1), digamos,$ $g_{1}()=.g_{2}()=.\exp ($.$) , e utilizando a SRR geral com o modelo de lei da potência$ inversa com estresse limiar (1.12) para o caso de uma única covariável $z$, isto é, fazendo $\left(z-\omega_{0}\right)=\left(\begin{array}{c}1 \\ -\log \left(x_{i}-\omega_{0}\right)\end{array}\right), \alpha^{T}=\left(\begin{array}{ll}\beta_{0} & \beta_{1}\end{array}\right)$, obtemos:

$$
\begin{aligned}
h(t \mid x) & =\exp \left(\alpha^{T}\left(z-\omega_{0}\right)\right) h_{0}\left(\exp \left(\alpha^{T}\left(z-\omega_{0}\right)\right) t ; \phi\right) \\
& =\exp \left(\beta_{0}-\beta_{1} \log \left(x_{i}-\omega_{0}\right)\right) h_{0}\left(\exp \left(\beta_{0}-\beta_{1} \log \left(x_{i}-\omega_{0}\right)\right) t ; \phi\right) \\
& =\exp \left(\beta_{0}-\beta_{1} \log \left(x_{i}-\omega_{0}\right)\right) \phi\left(\exp \left(\beta_{0}-\beta_{1} \log \left(x_{i}-\omega_{0}\right)\right) t\right)^{\phi-1} \\
& =\phi \lambda(\lambda t)^{\phi-1}=\phi \lambda^{\phi} t^{\phi-1},
\end{aligned}
$$

a qual é a função de risco de uma variável aleatória com uma distribuição Weibull com parâmetros de escala e forma $\lambda$ e $\phi$, respectivamente (veja equação (3.5)). 


\subsection{Métodos de estimação}

\subsubsection{Estimação via máxima verossimilhança}

Considerando os dados de $m$ níveis de estresse e $n_{i}$ observações com um esquema de censura à direita, como descrito no Apêndice A, segue de (6.17) e (3.6) que o núcleo da função de verossimilhança para os parâmetros $\beta_{0}, \beta_{1}, \omega_{0}$ e $\phi$, sob o $i$ - ésimo nível de estresse $x_{i}$, é dado por:

$$
\begin{gathered}
l=\log L\left(\beta_{0}, \beta_{1}, \omega_{0}, \phi\right) \alpha \sum_{i=1}^{m} \sum_{j=1}^{n_{i}}\left\{\delta_{i j}\left[\log \phi+\phi\left(-\beta_{0}+\beta_{1} \log \left(x_{i}-\omega_{0}\right)\right)+(\phi-1) \log t_{i j}\right]\right\}- \\
{\left[\exp \left(-\beta_{0}+\beta_{1} \log \left(x_{i}-\omega_{0}\right)\right) t_{i j}\right]^{\phi}}
\end{gathered}
$$

Os estimadores de máxima verosimilhança podem ser obtidos por maximização direta de (3.7), ou resolvendo o sistema de equações não lineares $\frac{\partial \log L(\psi)}{\partial \psi}=0$, onde $\psi=$ $\left(\beta_{0}, \beta_{1}, \omega_{0}, \phi\right)$. Inferências para o vetor de parâmetros $\psi$ podem ser baseadas nas propriedades de grandes amostras dos EMV's (veja Apêndice A), isto é:

$$
I^{1 / 2}(\hat{\psi})(\hat{\psi}-\psi) \stackrel{D}{\longrightarrow} N\left(0, I_{4}\right),
$$

onde $I^{1 / 2}(\widehat{\varphi})$ é a matriz de informação observada $\varphi$ calculada nos EMV's e $I_{4}$ é a matriz identidade de dimensão quatro.

\subsubsection{Um estudo de simulação}

Para verificar o comportamento da teoria assintótica para tamanhos de amostras pequenos ou moderados, um estudo de simulação de pequena escala foi realizado, com o objetivo de examinar as probabilidades de cobertura dos intervalos de confiança para os parâmetros do modelo, obtidos via (3.8). Foram geradas amostras de acordo com um estudo baseado em quatro níveis de covariáveis, $x=10,20,30,40 \operatorname{com} n=10,30,70,150$ observações não censuradas em cada nível. Para os tempos de vida, consideramos uma distribuição Weibulll com parâmetro de forma $\phi$, e parâmetro de escala $\lambda$ dado pela relação estresse resposta-geral (1.12), $\lambda_{i}=\exp \left\{-\beta_{0}+\beta_{1} \log \left(x_{i}-\omega_{0}\right)\right\}$. Os valores dos parâmetros foram fixados em $\beta_{0}=5, \beta_{1}=3, \omega_{0}=8$ e $\phi=3$. Um total de 1000 amostras foi gerado para cada caso. A Tabela (4) mostra a variação em cobertura na convergência de intervalos 
de confiança nominais de $95 \%$, de acordo com os tamanhos de amostras. O intervalo de confiança de $95 \%$ para a probabilidade de cobertura nominal de 0.95 , baseado em uma amostra de mil observações, é dado por $(0.937,0.964)$. Se um intervalo de confiança tem cobertura exata de 0.95 , aproximadamente $95 \%$ das coberturas observadas deveriam estar dentro desses limites. Observe que a cobertura dos intervalos de confiança é muito reduzida para tamanhos de amostras pequenos ou moderados. Para o parâmetro de forma da Weibull $\phi$ essa cobertura é ainda pior mesmo para tamanhos de amostras grandes. Os resultados indicaram evidências da necessidade de procedimentos mais adequados para esses casos. Por esta razão desenvolvemos uma análise Bayesiana para o modelo.

Tabela 3.2: Probabilidades de cobertura de intervalos de confiança nominais de 95\%.

\begin{tabular}{ccccc}
\hline \hline$n$ & $\beta_{0}$ & $\beta_{1}$ & $\omega_{0}$ & $\phi$ \\
\hline 10 & 0.90 & 0.89 & 0.87 & 0.82 \\
30 & 0.91 & 0.92 & 0.90 & 0.85 \\
70 & 0.93 & 0.95 & 0.92 & 0.87 \\
150 & 0.96 & 0.95 & 0.94 & 0.92 \\
\hline \hline
\end{tabular}

\subsubsection{O método MCMC}

Supondo que uma distribuição a priori para $\psi=\left(\beta_{0}, \beta_{1}, \omega_{0}, \phi\right)$ seja dada por $\pi(\psi)=$ $\pi\left(\beta_{0}\right) \pi\left(\beta_{1}\right) \pi\left(\omega_{0}\right) \pi(\phi)$, e considerando a função de verosimilhança $L\left(\beta_{0}, \beta_{1}, \omega_{0}, \phi\right)=$ $\exp (l)$ dada em (3.7), a densidade a posteriori conjunta para $\psi$ pode ser escrita através de (6.26) como:

$$
\begin{gathered}
\pi\left(\beta_{0}, \beta_{1}, \rho, \phi \mid \text { dados }\right) \alpha \prod_{i=1}^{m} \prod_{j=1}^{n_{i}}\left\{\left[\phi \lambda_{i}^{\phi} t_{i j}^{\phi-1}\right]^{\delta_{i j}} \exp \left(-\left(\lambda_{i} t_{i j}\right)^{\phi}\right)\right. \\
\left.p\left(\beta_{0}\right) p\left(\beta_{1}\right) p\left(\omega_{0}\right) p(\phi)\right\}
\end{gathered}
$$

Consideremos a mesma reparametrização $\omega_{0}=x_{1}-\exp (\rho)$ usada no caso exponencial, proposta por Davison e Hinkley (1997), e distribuições a priori da forma $p\left(\beta_{0}, \beta_{1}, \rho\right) \backsim$ $N\left(\mu_{i}, \sigma_{i}^{2}\right)$, com $\mu_{i}=0$, e $p(\phi) \sim \operatorname{gama}(a, b)$, esta última motivada pelo fato de que o parâmetro de forma $\phi$ de um modelo Weilbull é não negativo. Os valores dos hiper- 
parâmetros foram escolhidos de modo a representar prioris localmente não-informativas. Assim, substituindo as densidades a priori acima em (3.9), obtemos as posterioris condicionais

$$
\begin{gathered}
\pi\left(\beta_{0} \mid \beta_{1}, \rho, \phi\right) \alpha \exp \left\{\sum_{i=1}^{m} \sum_{j=1}^{n_{i}}-\delta_{i j} \phi \beta_{0}+\left(-\left(\lambda_{i} t_{i j}\right)^{\phi}\right)-\frac{\beta_{0}^{2}}{2 \sigma_{1}^{2}}\right\}, \\
\pi\left(\beta_{1} \mid \beta_{0}, \rho, \phi\right) \alpha \exp \left\{\sum_{i=1}^{m} \sum_{j=1}^{n_{i}} \delta_{i j} \phi\left(\beta_{1} \log \left(x_{i}-x_{1}+\exp (\rho)\right)\right)-\left(\left(\lambda_{i} t_{i j}\right)^{\phi}\right)-\frac{\beta_{1}^{2}}{2 \sigma_{2}^{2}}\right\}, \\
\pi\left(\rho \mid \beta_{1}, \beta_{0}, \phi\right) \alpha \exp \left\{\sum_{i=1}^{m} \sum_{j=1}^{n_{i}} \delta_{i j} \phi\left(\beta_{1} \log \left(x_{i}-x_{1}+\exp (\rho)\right)\right)-\left(\left(\lambda_{i} t_{i j}\right)^{\phi}\right)-\frac{\rho^{2}}{2 \sigma_{3}^{2}}\right\}, \\
\pi\left(\phi \mid \beta_{1}, \beta_{0}, \rho\right) \alpha \exp \left\{\sum_{i=1}^{m} \sum_{j=1}^{n_{i}} \delta_{i j}\left[\phi \lambda_{i}^{\phi} t_{i j}^{(\phi-1)}\right]-\left(\left(\lambda_{i} t_{i j}\right)^{\phi}\right)-b \phi+(a-1) \log \phi\right\}
\end{gathered}
$$

Observamos que tais posterioris condicionais não pertencem a nenhuma família paramétrica de distribuições conhecidas. Desta forma, amostrar valores diretamente das mesmas é, em princípio, impossível. Assim, utilizamos o método alternativo de rejeição adaptativo (ARS), descrito no Apêndice B, uma vez que todas as posterioris condicionais são log-côncavas, conforme verificado através de (6.27).

\subsection{Exemplo numérico}

Neste exemplo, consideramos os tempos de falha em horas de 70 fibras Kevlar 49 submetidas a $m=3$ níveis de estresse, $x_{i}=23.4,25.5$ e 27,6 MPa para $i=1, \ldots, 3$, com $n_{i}=21,25$ e 24 observações em cada nível. No primeiro nível, 11 das observações foram censuradas em 41000 horas. Os dados foram retirados da Tabela 2 de Smith (1991).

Em aplicações, uma maneira de avaliar a suposição de que o parâmetro de forma seja dependente de covariáveis, sem lançar mão de métodos analíticos, é através de diagnósticos gráficos. Em situações em que a variância é a mesma para todos os níveis de estresse, espera-se observar retas paralelas nos gráficos de $\log (-\log (S(t)))$ versus $\log (t)$, em que 
$S(t)$ é a função de sobrevivência. Podemos observar na Figura 3.2 relações lineares e paralelas, o que indica que $S(t)$ é bem ajustada pelo modelo Weibull com parâmetro de forma constante (veja Seção 1.8).

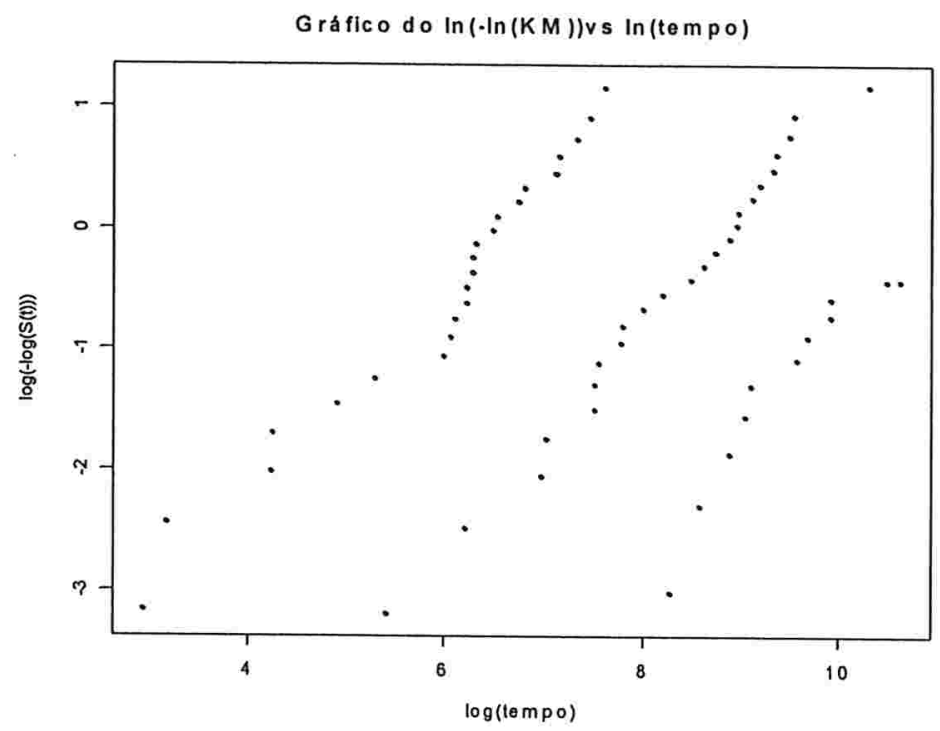

Figura 3.2: Gráfico de $\log (-\log (S(t)))$ por $\log (t)$

Risco empírico acumulado

As estimativas de máxima verossimilhança e MCMC, assim como seus intervalos de confiança e credibilidade obtidos pelo ajuste do modelo Weibull, são apresentados na Tabela (3.2). Os valores estimados para os parâmetros foram baseados em três amostras simuladas com 54000 iterações cada e um período de "burn-in" de 4000 iterações. Para cada cadeia, foram tomados valores de 7 em 7, obtendo-se assim uma cadeia de tamanho 21429 . Este critério de seleção foi sugerido pelo diagnóstico de convergência de Raftery e Lewis (1992). Através do diagnóstico de convergência de Gelman-Rubin, obtivemos valores de $\hat{\mathrm{R}}=1.01 ; 1.02 ; 1.0001,1.01$ para $\beta_{0}, \beta_{1}, \phi$ e $\omega_{0}$, respectivamente. Tais valores trouxeram fortes indícios de convergência para a densidade a posteriori dos parâmetros. Utilizando esse número de iterações, os quatro diagnósticos implementados no CODA indicaram a existência de convergência das amostras para cada um dos parâmetros. Podemos dizer que os resultados das análises clássica e Bayesiana foram próximos, com intervalos de confiança mais fechados para a análise clássica, como era esperado. Outros valores também foram considerados para os hiperparâmetros das densidades a priori. Os resultados obtidos foram muito similares a estes, e portanto não são apresentados. O parâmetro de estresse limiar foi estimado via MCMC. Observe que o ajuste parece 
amplamente satisfatório. A Figura (3.3) mostra o comportamento das trajetórias das cadeias geradas e as respectivas densidades a posteriori.

Tabela 3.2: Estimativas clássica e Bayesiana e IC de $95 \%$ para os parâmetros

\begin{tabular}{lcccc}
\hline \hline Procedimento & $\hat{\beta}_{0}$ & $\hat{\beta}_{1}$ & $\hat{\omega}_{0}$ & $\hat{\phi}$ \\
\hline EMV & 7.99 & 0.48 & 22.01 & 0.69 \\
Int. confiança & $(7.60 ; 8.99)$ & $(0.06 ; 1.24)$ & $(21.76 ; 22.687)$ & $(0.565 ; 0.799)$ \\
MCMC & 8.62 & 0.56 & 22.60 & 0.66 \\
Int. credibilidade & $(7.94 ; 9.49)$ & $(0.051 ; 1.32)$ & $(21.99 ; 22.94)$ & $(0.534 ; 0.812)$ \\
\hline \hline
\end{tabular}

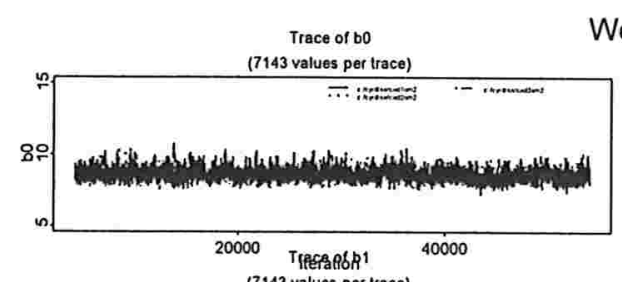

Weibull Kernel density for bo
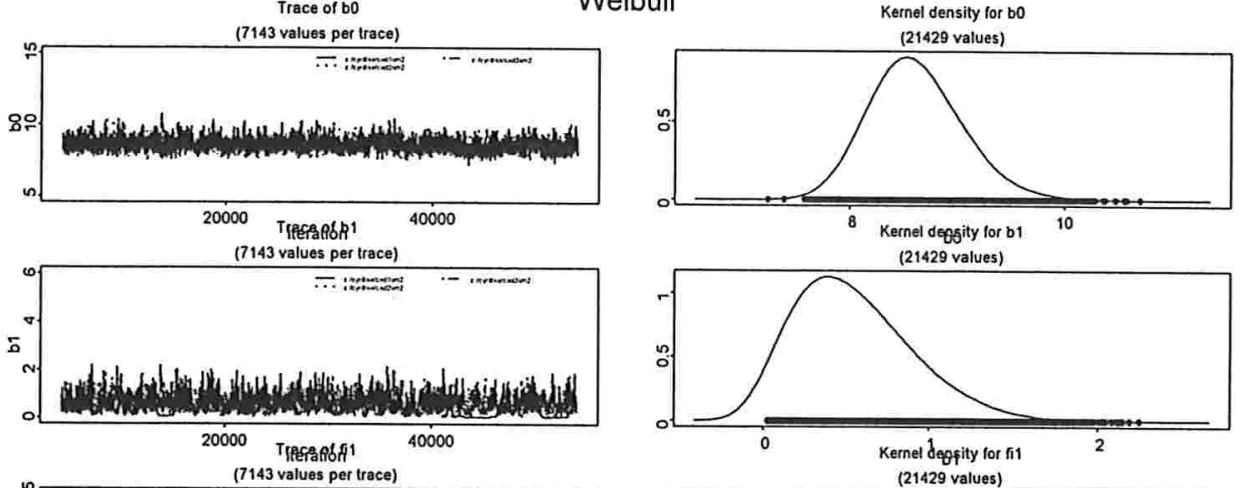

(21429 values)
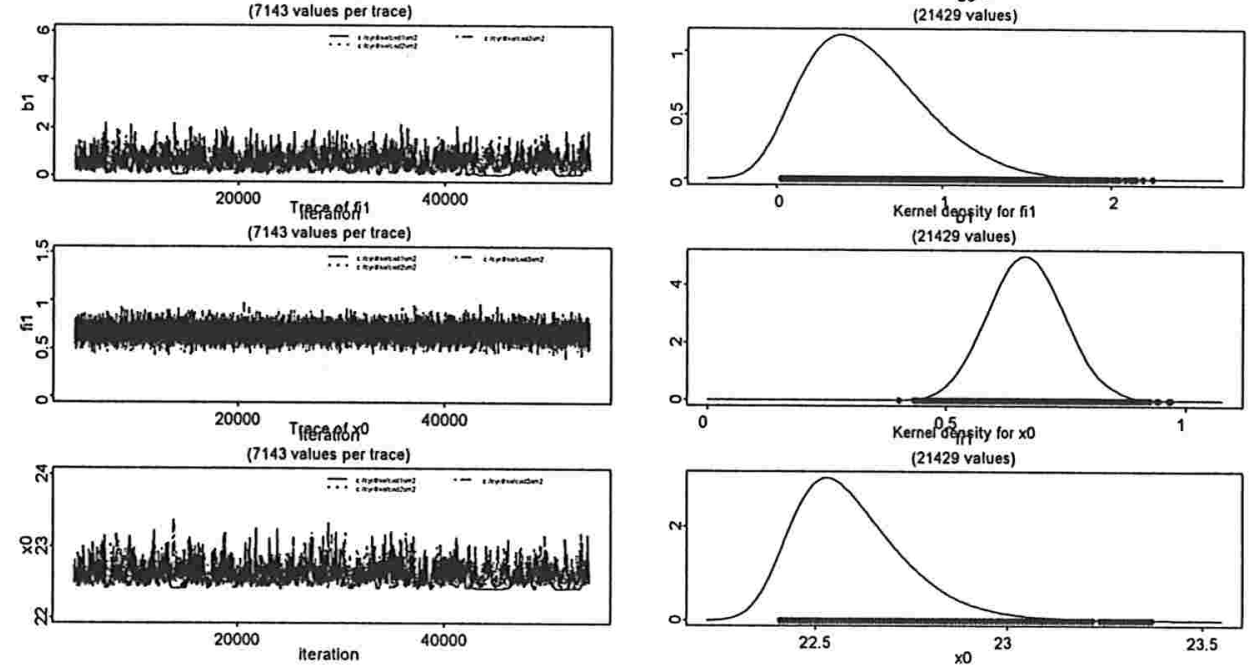

Figura 3.3: Gráficos das posterioris marginais e a história das iterações do algoritmo ARS

\subsection{Conclusões}

Neste capítulo, propusemos um modelo de riscos híbrido que, além de um incluir um estresse limiar, contém como casos particulares os modelos de riscos mais conhecidos em análise de sobrevivência e confiabilidade, isto é, os modelos de riscos proporcionais 
de Cox (Cox, 1972), o modelo de sobrevivência acelerado (Prentice, 1978) e o modelo híbrido (Etezadi-Amoli, 1987). Para a representação da função de risco base no modelo, adotamos uma abordagem paramétrica baseada na distribuição Weibull.

Verificamos o comportamento da teoria assintótica para tamanhos de amostras pequenos ou moderados, através de um estudo de simulação de pequena escala, e examinamos assim as probabilidades de cobertura dos intervalos de confiança para os parâmetros do modelo. Os resultados indicaram que os procedimentos usuais de inferência clássica podem não ser muito precisos para tamanhos de amostras pequenos ou moderados. Por esse motivo, para a estimação dos parâmetros consideramos, além da abordagem clássica, uma análise Bayesiana em que os valores das posterioris condicionais foram amostrados através do algoritmo ARS, o qual mostrou-se uma ferramenta bastante útil em problemas de estimação Bayesiana.

Através de técnicas gráficas, observamos para um conjunto de dados reais que a função de sobrevivência $S(t)$ poderia ser ajustada pelo modelo Weibull com parâmetro de forma constante. Para esse mesmo conjunto de dados, ambos os procedimentos de estimação resultaram em estimativas similares.

De uma maneira geral, o objetivo de mostrar a aplicabilidade do modelo de riscos híbrido com estresse limiar, na presença de covariáveis e de observações censuradas, foi atingido.

No entanto, restou ainda um ponto importante a ser tratado: o que devemos ou podemos fazer quando as técnicas gráficas indicarem, em um certo conjunto de dados, relações lineares porém não paralelas, mostrando que um modelo paramétrico pode ser utilizado, mas com o parâmetro de forma não constante, ou dependente dos níveis de estresse?

Modelos estatísticos em que o parâmetro de forma depende de covariáveis podem ser encontrados, por exemplo, em Nelson (1984), Meeter and Meeker (1994) e, do ponto de vista de modelos de riscos, em Louzada-Neto (1997). Entretanto, a presença de um parâmetro de estresse limiar não é considerada nesses artigos. Portanto, modelos estatísticos nos quais o parâmetro de forma depende dos níveis da covariável e de parâmetros de estresse limiar foram investigados no contexto de modelos de riscos no capítulo seguinte (4). 


\section{Capítulo 4}

\section{Um Modelo Geral de Riscos Híbrido com Estresse Limiar}

Neste capítulo propomos um modelo ainda mais geral de riscos híbrido com estresse limiar, o qual contém aquele proposto no Capítulo 3. Consequentemente, tanto o modelo de riscos proporcionais de Cox ( $\mathrm{PH}$ ) como o de sobrevivência acelerado (AFT) são casos particulares, e incluímos ainda um estresse limiar. Para a representação da função de risco base no modelo, adotamos uma abordagem paramétrica baseada agora nas distribuições gama-generalizada e log-logística.

Para a estimação dos parâmetros, consideramos as abordagens clássica e Bayesiana. Através de um estudo de simulação, mostramos as probabilidades de cobertura dos intervalos de confiança para os parâmetros através da teoria assintótica usual. Ilustramos a metodologia com um conjunto de dados simulados e outro de dados reais de modelos AFT.

\subsection{Apresentação do modelo}

O modelo geral de riscos híbrido com estresse limiar considerado neste capítulo é dado por

$$
h(t \mid z)=g_{1}\left(\alpha_{1}^{T}\left(z-\omega_{0}\right)\right) \phi\left[g_{1}\left(\alpha_{1}^{T}\left(z-\omega_{0}\right)\right) t\right]^{\phi-1} h_{0}\left[\left(g_{2}\left(\alpha_{2}^{T}\left(z-\omega_{0}\right)\right) t\right)^{\phi}\right],
$$


onde, como no capítulo anterior, $g_{1}($.$) e g_{2}($.$) são funções monótonas positivas conhecidas$ que assumem o valor 1 quando seus argumentos são zero, isto é, $g_{1}(0)=1=g_{2}(0), z$ é um vetor de covariáveis, $\alpha_{1}, \alpha_{2}$ e $\phi$ são vetores de parâmetros de regressão desconhecidos, $\omega_{0}$ é o vetor de parâmetros desconhecidos de estresses limiares, com $z_{i}>\omega_{0_{i}}$ para todo $i$, e $h_{0}($.$) é a função de risco base.$

A vantagem desta formulação é admitir várias classes de modelos de riscos como casos particulares. Quando $\alpha_{1}=\alpha_{2}$ e $\phi=1$, temos o modelo de falha acelerada (AFT) (Prentice, 1978) e, para $\alpha_{2}=0$ e $\phi=1$, o modelo de riscos proporcionais de Cox (PH) (Cox, 1972), mas agora com o estresse limiar $\omega_{0}$. O modelo (4.1) reduz-se ao modelo usual de riscos híbrido quando $\omega_{0}=0$ e $\phi=1$ (Etezadi-Amoli e Ciampi, 1987).

O modelo (4.1) é semi-paramétrico se a forma da função de risco base $h_{0}$ (.) é determinada não parametricamente a partir dos dados. Do ponto de vista paramétrico, supondo $g_{1}()=.g_{2}()=.g(.) \mathrm{e}$

$$
h_{0}(u ; k)=\frac{\Gamma(k)^{-1} u^{k-1} e^{-u}}{1-I(k ; u)}
$$

onde $I(k ; u)=\Gamma(k)^{-1} \int_{0}^{u} a^{k-1} e^{-a} d a$ é uma função gama incompleta (Lawless, 1982), obtemos uma rica classe de modelos paramétricos PH/AFT com estresse limiar. Sob estas restrições, o modelo (4.1) corresponde à função de risco de uma variável aleatória com uma distribuição gama-generalizada com três parâmetros (Lawless, 1982), um dos quais, $\lambda=g\left(\alpha^{T}\left(z-\omega_{0}\right)\right)$, dependendo de covariáveis, e com um estresse limiar, $\omega_{0}$. Fazendo $k=1$ em (4.2), recaímos em uma função de risco de um modelo Weibull considerada no capítulo anterior. Os modelos mais importantes utilizados em confiabilidade ou análise de sobrevivência estão incluídos nesta família paramétrica: a exponencial $(\phi=k=1)$, Weibull $(k=1)$, gama $(\phi=1)$ e log-normal $(k \rightarrow \infty)$. Além disso, para

$$
h_{0}(u)=\frac{1}{1+u}
$$

obtemos a distribuição log-logística com o parâmetro de escala dependente de covariáveis (Kalbfleisch and Prentice, 1980). 
Tabela 4.1: Relações entre os modelos de riscos supondo $g_{1}=g_{2}, \lambda_{1}=g\left(\alpha_{1}^{T}\left(z-\omega_{0}\right)\right)$ e $\lambda_{2}=g\left(\alpha_{2}^{T}\left(z-\omega_{0}\right)\right) . \quad \lambda^{*}, \lambda_{1}^{*}$ e $\lambda_{2}^{*}$ são iguais a $\lambda, \lambda_{1}$ e $\lambda_{2}$, respectivamente, com $\omega_{0}$ igual a 0.

\begin{tabular}{lcc}
\hline \hline Modelo & Restrição & Risco \\
\hline Geral PH/AFT com " $\omega_{0} "$ & - & $\lambda_{1} \phi\left(\lambda_{1} t\right)^{\phi-1} h_{0}\left(\left(\lambda_{2} t\right)^{\phi}\right)$ \\
AFT com " $\omega_{0} "$ & $\alpha_{1}=\alpha_{2} \Rightarrow\left(\lambda_{1}=\lambda_{2}=\lambda\right), \phi=1$ & $\lambda h_{0}(\lambda t)$ \\
PH com " $\omega_{0} "$ & $\alpha_{2}=0, \phi=1$ & $\lambda_{1} h_{0}(t)$ \\
PH/AFT & $\omega_{0}=0, \phi=1$ & $\lambda_{1}^{*} h_{0}\left(\lambda_{2}^{*} t\right)$ \\
\hline \hline AFT & $\lambda_{1}=\lambda_{2}=\lambda, \omega_{0}=0, \phi=1$ & $\lambda^{*} h_{0}\left(\lambda^{*} t\right)$ \\
\hline \hline PH & $\alpha_{2}=0, \omega_{0}=0, \phi=1$ & $\lambda_{1}^{*} h_{0}(t)$ \\
\hline \hline
\end{tabular}

\subsection{O caso da distribuição gama-generalizada}

A distribuição gama-generalizada foi introduzida por Stacy (1962) e é caracterizada por três parâmetros $\lambda, k$ e $\phi$, todos positivos. Essa distribuição despertou o interesse de vários pesquisadores pelo fato de representar uma família paramétrica que possui como casos particulares as distribuições mais utilizadas em análise de dados de tempos de vida e em confiabilidade. Apesar de sua importância, no entanto, ela possui o incoveniente de apresentar dificuldades operacionais na análise estatística do modelo. Hager e Bain (1970) obtiveram as equações de máxima verossimilhança para o modelo e notaram que o método de Newton-Raphson não funciona bem na solução da equação. Observaram ainda que os EMV's deixam de convergir quando se tem amostras maiores do que 200. Vários outros autores propuseram reparametrizações no modelo para que os EMV's pudessem ser facilmente encontrados (veja, por exemplo, Prentice (1974), Farewell e Prentice (1977) e Lawles (1980)).

As funções de densidade de probabilidade, de sobrevivência e de risco para o modelo gama-generalizado são dadas, respectivamente, por

$$
\begin{gathered}
f(t)=\frac{\lambda \phi}{\Gamma(k)}(\lambda t)^{\phi k-1} \exp \left\{-(\lambda t)^{\phi}\right\} ; \quad t, k, \phi, \lambda>0, \\
S(t)=1-I\left[k,(\lambda t)^{\phi}\right]
\end{gathered}
$$




$$
h(t)=\frac{\phi \lambda^{\phi k} t^{\phi k-1} \exp \left\{-(\lambda t)^{\phi}\right\}(\Gamma(k))^{-1}}{1-I\left[k,(\lambda t)^{\phi}\right]},
$$

em que $I[k, x]$ é a função gama incompleta dada por

$$
I[k, x]=\frac{1}{\Gamma(k)} \int_{0}^{x} u^{(k-1)} e^{-u} d u .
$$

Como casos particulares da distribuição gama generalizada, temos as seguintes distribuições:

- Exponencial $\longrightarrow k=1, \phi=1$;

- Weibull $\longrightarrow k=1$;

- Gama $\longrightarrow \phi=1$;

- Log-normal $\longrightarrow k \rightarrow \infty$;

- Qui-quadrado $\longrightarrow k=1, \phi=n / 2, \lambda=2$.

Considerando $h_{0}$ como em (4.2), supondo $g_{1}()=.g_{2}($.$) no modelo (4.1), digamos,$ $g_{1}()=.g_{2}()=.\exp ($.$) , e utilizando a SRR geral com o modelo de lei da potência in-$ versa com estresse limiar (1.12) para o caso de uma única covariável $z$, isto é, fazendo

$$
\begin{aligned}
& \left(z-\omega_{0}\right)=\left(\begin{array}{c}
1 \\
-\log \left(x_{i}-\omega_{0}\right)
\end{array}\right), \alpha^{T}=\left(\begin{array}{ll}
\beta_{0} & \beta_{1}
\end{array}\right) \text {, obtemos: } \\
& h(t \mid z)=\exp \left(\alpha^{T}\left(z-\omega_{0}\right)\right) \phi\left[g_{1}\left(\alpha^{T}\left(z-\omega_{0}\right)\right) t\right]^{\phi-1} h_{0}\left[\left(g_{2}\left(\alpha^{T}\left(z-\omega_{0}\right)\right) t\right)^{\phi}\right] \\
& =\exp \left(\beta_{0}-\beta_{1} \log \left(x_{i}-\omega_{0}\right)\right) \phi\left[\exp \left(\beta_{0}-\beta_{1} \log \left(x_{i}-\omega_{0}\right)\right) t\right]^{\phi-1} \frac{\Gamma(k)^{-1}(\lambda t)^{\phi(k-1)} e^{-(\lambda t)^{\phi}}}{1-I\left(k ;(\lambda t)^{\phi}\right)} \\
& =\lambda \phi(\lambda t)^{\phi-1}(\lambda t)^{\phi(k-1)} e^{-(\lambda t)^{\phi}} \frac{\Gamma(k)^{-1}}{1-I\left(k ;(\lambda t)^{\phi}\right)} \\
& =\phi \lambda^{\phi k} t^{\phi k-1} \exp \left[-(\lambda t)^{\phi}\right] \frac{\Gamma(k)^{-1}}{1-I\left(k ;(\lambda t)^{\phi}\right)},
\end{aligned}
$$

a qual é a função de risco de uma variável aleatória com uma distribuição gama generalizada com parâmetros de escala $\lambda, \phi$ e parâmetro de forma $k$ (Lawless, 1982). 


\subsection{Métodos de estimação}

\subsubsection{Estimação via máxima verossimilhança}

Considerando os dados de $m$ níveis de estresse e $n_{i}$ observações com um esquema de censura à direita, assim como no caso Weibull, segue-se de (6.17) e (4.8) que o núcleo da função de verossimilhança para os parâmetros $\beta_{0}, \beta_{1}, \omega_{0}, \phi$ e $k$, sob o $i$-ésimo nível de estresse $x_{i}$, é dado por:

$$
\begin{gathered}
L\left(\beta_{0}, \beta_{1}, \omega_{0}, \phi, k\right) \alpha \prod_{i=1}^{m} \prod_{j=1}^{n_{i}}\left\{\left[\phi\left(e^{-\beta_{0}+\beta_{1} \log \left(x_{i}-\omega_{0}\right)}\right)^{\phi k} t_{i j}^{\phi k-1} \exp \left(-\left(\left(e^{-\beta_{0}+\beta_{1} \log \left(x_{i}-\omega_{0}\right)}\right) t_{i j}\right)^{\phi}\right)\right.\right. \\
\left.\left.\frac{\Gamma(k)^{-1}}{1-I\left(k ;\left(e^{-\beta_{0}+\beta_{1} \log \left(x_{i}-\omega_{0}\right)} t_{i j}\right)^{\phi}\right)}\right]^{\delta_{i j}}\left[1-I\left(k ;\left(e^{-\beta_{0}+\beta_{1} \log \left(x_{i}-\omega_{0}\right)} t_{i j}\right)^{\phi}\right)\right]\right\}
\end{gathered}
$$

Os estimadores de máxima verosimilhança podem ser obtidos, como no caso Weibull, por maximização direta de (4.9) ou resolvendo o sistema de equações não lineares $\partial \log L / \partial \varphi=$ 0 , onde $\varphi=\left(\beta_{0}, \beta_{1}, \omega_{0}, \phi, k\right)$. Entretanto, dificuldades de convergência para encontrar os EMV's dos parâmetros usualmente ocorrem em algoritmos numéricos, mesmo para grandes tamanhos de amostras (veja Harger e Bain, 1970).

Inferências para o vetor de parâmetros $\varphi$ podem ser baseadas nas propriedades de grandes amostras dos EMV's (veja Apêndice A), isto é:

$$
I^{1 / 2}(\widehat{\varphi})(\widehat{\varphi}-\varphi) \stackrel{D}{\longrightarrow} N\left(0, I_{5}\right)
$$

onde $I^{1 / 2}(\widehat{\varphi})$ é a matriz de informação observada $\varphi$ calculada nos EMV's e $I_{5}$ é a matriz identidade de dimensão cinco. Entretanto, métodos de grandes amostras baseados na aproximação normal para a distribuição dos EMV's são insatisfatórios, uma vez que a convergência para a distribuição normal limite é muito lenta (Lawless, 1982). Harger e Bain (1970) mostraram que aproximações normais podem ser muito grosseiras mesmo para tamanhos de amostras maiores do que 400. Vários autores propuseram reparametrizações no modelo de modo que a determinação dos EMV's pudesse ser feita de forma simples. (veja, por exemplo, Prentice (1974), Farewell e Prentice (1977) e Lawles (1980). Mesmo com o uso de reparametrizações, a estimação intervalar dada por (4.10) parece imprecisa, a menos que os tamanhos de amostras sejam muito grandes (Prentice, 1974). Como é usual em estudos de confiabilidade encontrar tamanhos de amostras pequenos ou moderados, 
desenvolvemos novamente uma abordagem Bayesiana do modelo.

\subsubsection{O método MCMC}

Consideramos as mesmas reparametrizações do caso Weibull (Capítulo 3), $\beta_{0}, \beta_{1}, \omega_{0}=$ $x_{1}-\exp (\rho)$, bem como as mesmas densidades a priori para $\beta_{0}, \beta_{1}, \rho$. Desde que os parâmetros $\phi$ e $k$ são não negativos, é apropriado usar uma distribuição a priori com suporte sobre os reais positivos. Consideramos aqui, por exemplo, uma distribuição gamma com parâmetros $(a, b)$ para $\phi$ e $(c, d)$ para $k$, respectivamente. Tal escolha mostrou-se apropriada através de uma análise de sensibilidade dos hiperparâmetros, a qual indicou resultados muito próximos para as estimações dos parâmetros para diferentes escolhas dos hiperparâmetros. Combinando as densidades a priori acima com (4.9), obtemos para $\beta_{0}, \beta_{1}, \rho, \phi$ e $k$ as densidades a posteriori

$$
\begin{aligned}
& \pi\left(\beta_{0}, \beta_{1}, \rho, \phi, k \mid \text { data }\right) \alpha \prod_{i=1}^{m} \prod_{j=1}^{n_{i}}\left\{\left[\phi \lambda_{i}^{\phi k} t_{i j}^{\phi k-1} \exp \left(-\left(\lambda_{i} t_{i j}\right)^{\phi}\right) \frac{\Gamma(k)^{-1}}{1-I\left(k ;\left(\lambda_{i} t_{i} j\right)^{\phi}\right)}\right]^{\delta_{i j}}\right. \\
& \left.\exp \left(-\frac{\beta_{0}^{2}}{2 \sigma_{1}^{2}}-\frac{\beta_{1}^{2}}{2 \sigma_{2}^{2}}-\frac{\rho^{2}}{2 \sigma_{3}^{2}}-b \phi-d k\right) \phi^{a-1} k^{c-1}\left[1-I\left(k ;\left(\lambda_{i} t_{i j}\right)^{\phi}\right)\right]\right\}
\end{aligned}
$$

onde $\lambda_{i}=\exp \left(-\beta_{0}+\beta_{1} \log \left(x_{i}-x_{1}+\exp (\rho)\right)\right)$.

As densidades condicionais completas para $\beta_{0}, \beta_{1}, \rho, \phi$ e $k$ são dadas, respectivamente, por

$$
\begin{gathered}
\pi\left(\beta_{0} \mid \beta_{1}, \rho, \phi, k\right) \alpha \quad \exp \left\{\sum_{i=1}^{m} \sum_{j=1}^{n_{i}}-\delta_{i j}\left[\phi k \beta_{0}-\left(\left(\lambda_{i} t_{i j}\right)^{\phi}\right)-\log \left(1-I\left(k ;\left(\lambda_{i} t_{i j}\right)^{\phi}\right)\right)\right]\right. \\
\left.+\log \left(1-I\left(k ;\left(\lambda_{i} t_{i j}\right)^{\phi}\right)\right)-\frac{\beta_{0}^{2}}{2 \sigma_{1}^{2}}\right\}, \\
\pi\left(\beta_{1} \mid \beta_{0}, \rho, \phi, k\right) \alpha \exp \left\{\sum _ { i = 1 } ^ { m } \sum _ { j = 1 } ^ { n _ { i } } \delta _ { i j } \left[\phi k\left(\beta_{1} \log \left(x_{i}-x_{1}+e^{\rho}\right)\right)-\left(\left(\lambda_{i} t_{i j}\right)^{\phi}\right)\right.\right. \\
\left.\left.-\log \left(1-I\left(k ;\left(\lambda_{i} t_{i j}\right)^{\phi}\right)\right)\right]+\log \left(1-I\left(k ;\left(\lambda_{i} t_{i j}\right)^{\phi}\right)\right)-\frac{\beta_{1}^{2}}{2 \sigma_{2}^{2}}\right\},
\end{gathered}
$$




$$
\begin{gathered}
\pi\left(\rho \mid \beta_{1}, \beta_{0}, \phi, k\right) \alpha \exp \left\{\sum _ { i = 1 } ^ { m } \sum _ { j = 1 } ^ { n _ { i } } \delta _ { i j } \left[\phi k\left(\beta_{1} \log \left(x_{i}-x_{1}+e^{\rho}\right)\right)-\left(\left(\lambda_{i} t_{i j}\right)^{\phi}\right)\right.\right. \\
\left.\left.-\log \left(1-I\left(k ;\left(\lambda_{i} t_{i j}\right)^{\phi}\right)\right)\right]+\log \left(1-I\left(k ;\left(\lambda_{i} t_{i j}\right)^{\phi}\right)\right)-\frac{\rho^{2}}{2 \sigma_{3}^{2}}\right\},(4.14) \\
\pi\left(\phi \mid \beta_{1}, \beta_{0}, \rho, k\right) \alpha \exp \left\{\sum _ { i = 1 } ^ { m } \sum _ { j = 1 } ^ { n _ { i } } \delta _ { i j } \left[\log \phi+\phi k \log \lambda_{i}+(\phi k-1) \log t_{i j}-\left(\left(\lambda_{i} t_{i j}\right)^{\phi}\right)\right.\right. \\
\left.\left.-\log \left(1-I\left(k ;\left(\lambda_{i} t_{i j}\right)^{\phi}\right)\right)\right]+\log \left(1-I\left(k ;\left(\lambda_{i} t_{i j}\right)^{\phi}\right)\right)-b \phi+(a-1) \log \phi\right\} \\
\pi\left(k \mid \beta_{0}, \beta_{1}, \rho, \phi\right) \alpha \exp \left\{\sum_{i=1}^{m} \sum_{j=1}^{n_{i}} \delta_{i j}\left[\phi k\left(\log \left(\lambda_{i}\right)+\log \left(t_{i j}\right)\right)+\frac{\Gamma(k)^{-1}}{1-I\left(k ;\left(\lambda_{i} t_{i j}\right)^{\phi}\right)}\right]\right. \\
\left.\left.+\log \left(1-I\left(k ;\left(\lambda_{i} t_{i j}\right)^{\phi}\right)\right)-(d k)+(c-1) \log k\right\} .16\right)
\end{gathered}
$$

e

Como podemos observar, as densidades condicionais para $\beta_{0}, \beta_{1}, \rho, \phi$ e $k$ não pertencem a nenhuma família paramétrica conhecida. Assim, para gerarmos amostras implementamos o algoritmo ARS (Gilks et al., 1995).

\subsection{O caso Log-logístico}

Uma outra distribuição que tem sido utilizada para modelar dados de sobrevivência é a distribuição log-logística. Embora seja menos utilizada em aplicações, ela possui a vantagem, assim como a Weibull e a exponencial, de ter expressões algébricas simples para as funções de sobrevivência e riscos. Uma variável aleatória não negativa $T$ segue uma distribuição log-logística com parâmetros $\lambda>0$ e $\phi>0$ se seu logaritmo $Y=\log (T)$ tem distribuição logística com função de densidade de probabilidade dada por (Klein e Moeschberger, 1997)

$$
f(y)=\frac{\exp (y-\alpha / \sigma)}{\sigma[1+\exp (y-\alpha / \sigma)]^{2}}
$$


em que $-\infty<\alpha<\infty$ e $\sigma>0$ são os parâmetros de locação e escala, respectivamente. Para obtermos as funções de sobrevivência $S(t)$ e de risco $h(t)$, devemos considerar uma reparametrização da forma $Y=\log (T)=\alpha+\sigma W$, onde $W \sim \operatorname{logística}(0,1)$. Assim, $\alpha=\log (\lambda)$ e $\sigma=\phi^{-1}$. Portanto, $S(t)$ e $h(t)$ são dadas, respectivamente, por:

$$
S(t)=\frac{1}{1+(\lambda t)^{\phi}}
$$

$\mathrm{e}$

$$
h(t)=\frac{\phi \lambda^{\phi} t^{\phi-1}}{1+\left((\lambda t)^{\phi}\right)} .
$$

Seja $h_{0}(u)$ dada por (4.3) e considere $g_{1}()=.g_{2}()=.\exp ($.$) em (4.1). Usando a$ relação estresse resposta-geral (1.12), temos que

$$
h(t \mid z)=\frac{\phi \lambda^{\phi} t^{\phi-1}}{1+(\lambda t)^{\phi}},
$$

a qual pode ser identificada como uma função de risco de uma variável aleatória com uma distribuição log-logística com parâmetro de escala $\lambda>0$ e parâmetro de forma $\phi>0$ (Lawless,1982). Observe que a função de risco apresenta comportamento unimodal.

Função de Sobrevivência Log-Logistica

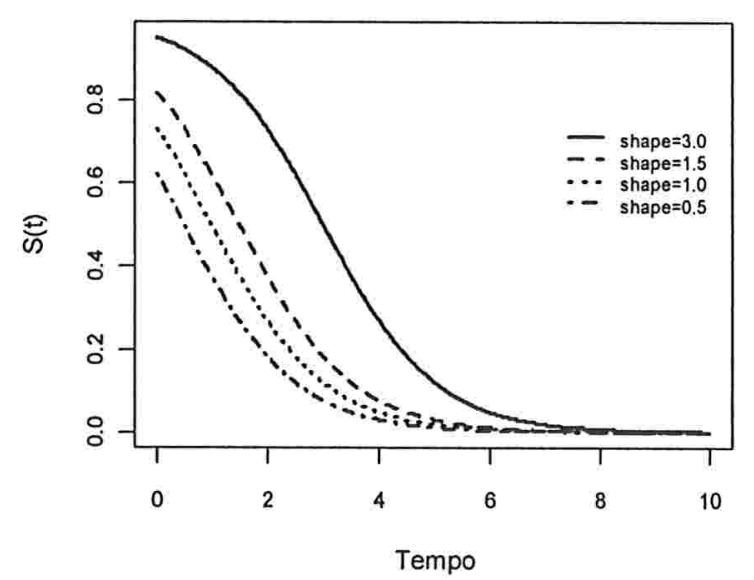

Função de Risco Log-Logistica

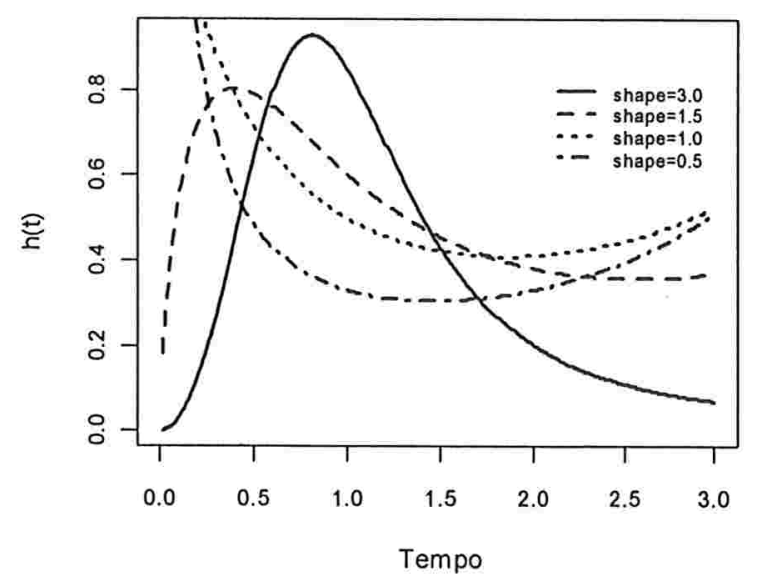

Figura 4.1: Gráfico das funções de sobrevivência e risco da distribuição log-logística para diferentes valores de $\phi$. 


\subsection{Métodos de estimação}

\subsubsection{Máxima verossimilhança}

Considerando os dados de $m$ níveis de estresse aleatorizados com um esquema de censura como o descrito no Apêndice A, decorre de (6.17) e (4.20) que o núcleo da função de verossimilhança para os parâmetros $\beta_{0}, \beta_{1}, \omega_{0}$ e $\phi$, sob o i-ésimo nível de estresse $x_{i}$, é dado por:

$$
L\left(\beta_{0}, \beta_{1}, \omega_{0}, \phi\right) \alpha \prod_{i=1}^{m} \prod_{j=1}^{n_{i}}\left[\frac{\phi \lambda_{i}^{\phi} t_{i j}^{\phi-1}}{1+\left(\lambda_{i} t_{i j}\right)^{\phi}}\right]^{\delta_{i j}}\left(\frac{1}{1+\left(\lambda_{i} t_{i j}\right)^{\phi}}\right) .
$$

Os EMV's de $\beta_{0}, \beta_{1}, \omega_{0}$ e $\phi$, bem como inferências para o vetor de parâmetros $\psi=\left(\beta_{0}, \beta_{1}, \omega_{0}, \phi\right)$, podem ser obtidos como antes, os quais levam a (Sen and Singer, 1993)

$$
I^{1 / 2}(\widehat{\psi})(\widehat{\psi}-\psi) \stackrel{D}{\longrightarrow} N\left(0, I_{4}\right)
$$

onde $I^{1 / 2}(\widehat{\psi})$ é a matriz de informação observada de $\psi$ calculada nos EMV's, e $I_{4}$ é a matriz identidade de dimensão quatro. Para verificar o comportamento da teoria assintótica para conjuntos de dados pequenos ou moderados, fizemos um estudo de pequena escala para examinar a probabilidade de cobertura dos intervalos de confiança para os parâmetros obtidos via (4.22). Geramos amostras em três níveis de covariáveis, $x=5,10,15$ com $n=$ 10, 30, 100 observações não censuradas em cada nível. Consideramos a distribuição loglogística (4.20) para os tempos de vida, com parâmetros de escala e forma $\lambda_{i}=\exp \left\{-\beta_{0}+\right.$ $\left.\beta_{1} \log \left(x_{i}-\omega_{0}\right)\right\}$, e $\phi$, respectivamente. Os valores dos parâmetros foram fixados em $\beta_{0}=2.5, \beta_{1}=2, \omega_{0}=4.8$, e $\phi=3$. Um total de 1000 amostras foi gerado para cada caso. A Tabela (4.2) mostra a variação em cobertura na convergência de intervalos de confiança nominais de $95 \%$, de acordo com os tamanhos de amostras. O intervalo de confiança de $95 \%$ para a probabilidade de cobertura nominal de 0.95 , baseado em uma amostra de 1000 observações, é dado por $(0.941,0.970)$. Se um intervalo de confiança tem cobertura exata de 0.95 , aproximadamente $95 \%$ das coberturas observadas deveriam estar dentro desses limites. Observe que a cobertura dos intervalos de confiança é muito reduzida para tamanhos de amostras pequenos ou moderados e torna-se ainda mais reduzida, mesmo para tamanhos de amostras grandes, para o parâmetro de forma $\phi$ da distribuição loglogística. Os resultados indicam evidências de que são necessários procedimentos mais adequados para esses casos. Por essa razão, desenvolvemos uma análise Bayesiana para 
o modelo. A Tabela (4.2) mostra a variação em cobertura dos intervalos de confiança nominais de $95 \%$, de acordo com o tamanho da amostra.

Tabela 4.2: Probabilidades de cobertura de intervalos de confiança nominais de $95 \%$.

\begin{tabular}{ccccc}
\hline \hline$n$ & $\beta_{0}$ & $\beta_{1}$ & $\omega_{0}$ & $\phi$ \\
\hline 10 & 0.88 & 0.89 & 0.86 & 0.83 \\
30 & 0.91 & 0.90 & 0.91 & 0.87 \\
100 & 0.95 & 0.96 & 0.94 & 0.91 \\
\hline \hline
\end{tabular}

\subsubsection{O método Bayesiano}

Considerando as mesmas reparametrizações para $\beta_{0}, \beta_{1}, \omega_{0}=x_{1}-\exp (\rho)$ e $\phi$, bem como as mesmas densidades a priori para $\beta_{0}, \beta_{1}, \rho$ e $\phi \sim \operatorname{Gama}(q, v)$, como na Seção 4.3, obtemos que a densidade a posteriori conjunta é dada por:

$\pi\left(\beta_{0}, \beta_{1}, \rho, \phi, \mid\right.$ data $) \alpha \prod_{i=1}^{m} \prod_{j=1}^{n_{i}}\left[\frac{\phi \lambda_{i}^{\phi} t_{i j}^{\phi-1}}{1+\left(\lambda_{i} t_{i j}\right)^{\phi}}\right]^{\delta_{i j}}\left(\frac{1}{1+\left(\lambda_{i} t_{i j}\right)^{\phi}}\right) \exp \left(-\frac{\beta_{0}^{2}}{2 \sigma_{1}^{2}}-\frac{\beta_{1}^{2}}{2 \sigma_{2}^{2}}-\frac{\rho^{2}}{2 \sigma_{3}^{2}}-v \phi\right) \phi^{q-1}$,

a qual pode ser escrita como

$$
\begin{aligned}
\pi\left(\beta_{0}, \beta_{1}, \rho, \phi, \mid \text { data }\right) \alpha \exp \{ & \left\{\sum_{i=1}^{m} \sum_{j=1}^{n_{i}} \delta_{i j} \log \left[\frac{\phi \lambda_{i}^{\phi} t_{i j}^{\phi-1}}{1+\left(\lambda_{i} t_{i j}\right)^{\phi}}\right]-\log \left(1+\left(\lambda_{i} t_{i j}\right)^{\phi}\right)\right. \\
& \left.-\left(\frac{\beta_{0}^{2}}{2 \sigma_{1}^{2}}+\frac{\beta_{1}^{2}}{2 \sigma_{2}^{2}}+\frac{\rho^{2}}{2 \sigma_{3}^{2}}+v \phi\right)+(q-1) \log \phi\right\},
\end{aligned}
$$

onde $\lambda_{i}=\exp \left\{-\beta_{0}+\beta_{1} \log \left(x_{i}-\omega_{0}\right)\right\}$. Portanto, as densidades condicionais completas para $\beta_{0}, \beta_{1}, \rho$ e $\phi$ são dadas, respectivamente, por

$$
\pi\left(\beta_{0} \mid \beta_{1}, \rho, \phi\right) \alpha \exp \left[\sum_{i=1}^{m} \sum_{j=1}^{n_{i}} \delta_{i j}\left(-\phi \beta_{0}\right)-\left(\delta_{i j}+1\right) \log \left(1+\left(\lambda_{i} t_{i j}\right)^{\phi}\right)-\frac{\beta_{0}^{2}}{2 \sigma_{1}^{2}}\right]
$$


$\pi\left(\beta_{1} \mid \beta_{0}, \rho, \phi\right) \alpha \exp \left[\sum_{i=1}^{m} \sum_{j=1}^{n_{i}} \delta_{i j}\left(\phi \beta_{1} \log \left(x_{i}-x_{1}+\exp (\rho)\right)\right)-\left(\delta_{i j}+1\right) \log \left(1+\left(\lambda_{i} t_{i j}\right)^{\phi}\right)-\frac{\beta_{\prime_{1}}^{2}}{2 \sigma_{2}^{2}}\right]$

$\pi\left(\rho \mid \beta_{0}, \beta_{1}, \phi\right) \alpha \exp \left\{\sum_{i=1}^{m} \sum_{j=1}^{n_{i}} \delta_{i j}\left[\phi \beta_{1} \log \left(x_{i}-x_{1}+\exp (\rho)\right)\right]-\left(\delta_{i j}+1\right) \log \left(1+\left(\lambda_{i} t_{i j}\right)^{\phi}\right)-\frac{\rho}{2 \sigma_{3}^{2}}\right\}$

e

$\pi\left(\phi \mid \beta_{0}, \beta_{1}, \rho\right) \alpha \exp \left\{\sum_{i=1}^{m} \sum_{j=1}^{n_{i}} \delta_{i j} \log \left[\phi \lambda_{i}^{\phi} t_{i j}^{\phi-1}\right]-\left(\delta_{i j}+1\right) \log \left(1+\left(\lambda_{i} t_{i j}\right)^{\phi}\right)-v \phi+(q-1) \log \phi\right\}$.

Podemos observar que as densidades condicionais para os parâmetros do modelo não pertencem a nenhuma família paramétrica conhecida, o que nos leva novamente à utilização do algoritmo ARS (Gilks et al, 1995), uma vez que todas as condicionais acima são log-côncavas.

\subsection{Exemplos}

\subsubsection{Dados simulados}

O estudo de simulação foi baseado em amostras geradas de uma distribuição log-logística. Usando a relação estresse resposta geral em (1.12), os valores dos parâmetros foram fixados em $\beta_{0}=2.5, \beta_{1}=2.0, \phi=3$ e $\omega_{0}=4.8$. Utilizamos três níveis de estresse $x=5,10,15$, com 20 observações não censuradas em cada nível. Esse conjunto de dados já foi estudado na Seção 1.8, onde vimos através de técnicas gráficas que o modelo log-logístico com parâmetro de forma constante ajusta bem os dados. 


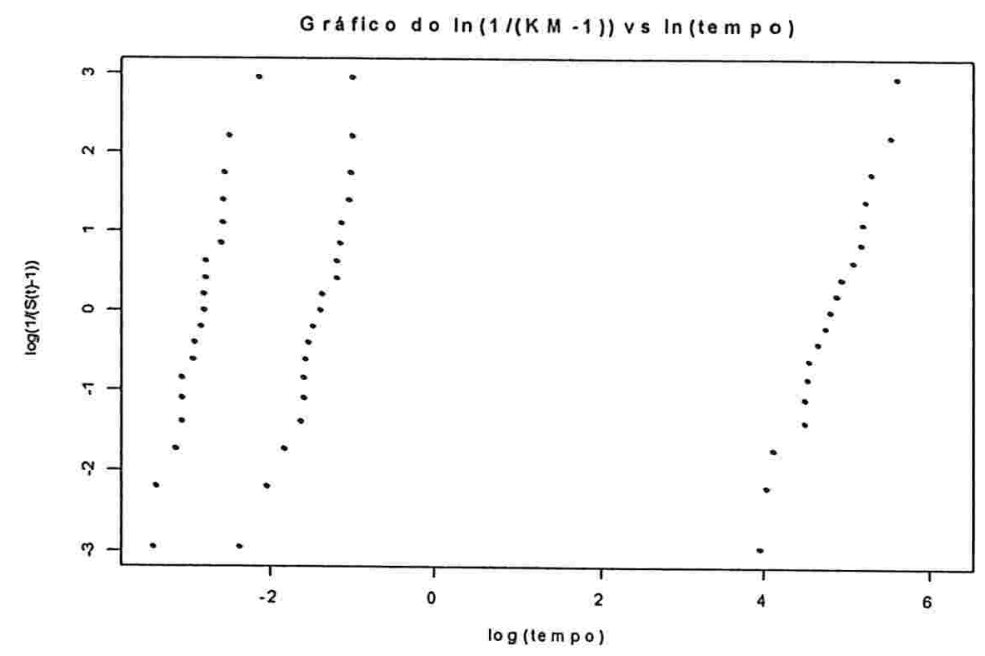

Figura 4.2: Gráfico de $\log \left(S(t)^{-1}-1\right)$ por $\log (t)$

A Tabela (4.3) mostra os estimadores dos parâmetros do modelo baseados em ambas as abordagens, clássica e Bayesiana com seus respectivos erros quadráticos médios. Para a inferência clássica, calculamos os intervalos de confiança de 95\% usando os EMV's dos parâmetros, e para a análise Bayesiana calculamos os intervalos de credibilidade de 95\% para os parâmetros do modelo pelo algoritmo de amostragem de Gibbs, os quais apresentaram resultados mais fechados, o que já era esperado, como mostrado na Tabela 4.2, pois o tamanho de amostra é pequeno.

Tabela 4.3 : Estimativas clássica e Bayesiana e seus respectivos intervalos de 95\%. (Médias e médias a posteriori).Dados simulados com

\begin{tabular}{|c|c|c|c|c|}
\hline Procedimento & $\hat{\beta}_{0}$ & $\hat{\beta}_{1}$ & $\omega_{0}$ & $\hat{\phi}$ \\
\hline \multirow[t]{2}{*}{ EMV } & 2.22 & 2.15 & 4.70 & 4.11 \\
\hline & $(E M Q=0.088)$ & $(E M Q=0.02)$ & $(\mathrm{EMQ}=0.01)$ & $(\mathrm{EMQ}=1.23)$ \\
\hline Int. confiança & $(1.265 ; 3.174)$ & $(1.714 ; 2.692)$ & $(4.40 ; 4.95)$ & $(3.24 ; 4.99)$ \\
\hline \multirow[t]{2}{*}{$\mathrm{MCMC}$} & 2.25 & 2.16 & 4.71 & 4.008 \\
\hline & $(\mathrm{EMQ}=0.06)$ & $(\mathrm{EMQ}=0.02)$ & $(\mathrm{EMQ}=0.01)$ & $(\mathrm{EMQ}=0.98)$ \\
\hline Int. credibilidade & $(2.30 ; 3.26)$ & $(2.02 ; 2.62)$ & $(4.45 ; 4.87)$ & $(3.78 ; 4.61)$ \\
\hline
\end{tabular}




\subsubsection{Dados reais 1}

Neste exemplo, consideramos os tempos de vida de fibras de carbono com diferentes comprimentos, obtidos na "University of Surrey" por " Dr. Mark Priest" e representados na Tabela 3 de Smith (1991). O efeito do tamanho descreve a dependência esperada da força do material em relação ao seu comprimento, assim é natural tomar o comprimento como uma covariável. Para esse tipo de conjunto de dados, a distribuição Weibull é amplamente utilizada. Neste trabalho, consideramos $m=3$ níveis de estresse; $x_{i}=$ $10,20,50 \mathrm{~mm}$ para $i=1, \ldots, 3, \operatorname{com} n_{i}=63,70$ e 62 observações não censuradas em cada nível. Obtemos uma estimativa $\hat{k}=1.05(0.43,3.27)$, o que indica que um modelo Weibull é apropriado para ajustar os dados.

Em aplicações, uma maneira de avaliar a suposição de que o parâmetro de forma seja dependente de covariáveis, sem lançar mão de métodos analíticos, é através de diagnósticos gráficos, como mostrado na seção 1.8. Como vimos, em situações em que a variância é a mesma para todos os níveis de estresse, espera-se observar retas paralelas nos gráficos de $\log (-\log (S(t)))$ versus $\log (t)$, em que $S(t)$ é a função de sobrevivência. Podemos observar na Figura 4.3 relações lineares e paralelas, o que indica que $S(t)$ é bem ajustada pelo modelo Weibull com parâmetro de forma constante (veja Seção 1.8). Entretanto, ajustamos um modelo Weibull com um parâmetro de forma independente para cada nível da covariável $x_{i}$ e observamos mudanças não significativas para $\phi$; mais precisamente, os resultados obtidos foram $\hat{\phi}_{1}=5.43, \hat{\phi}_{2}=5.52$ e $\hat{\phi}_{3}=5.65$, respectivamente, para os três níveis de estresse. Tais resultados indicam que um parâmetro de forma constante para esse conjunto de dados é de fato apropriado. 


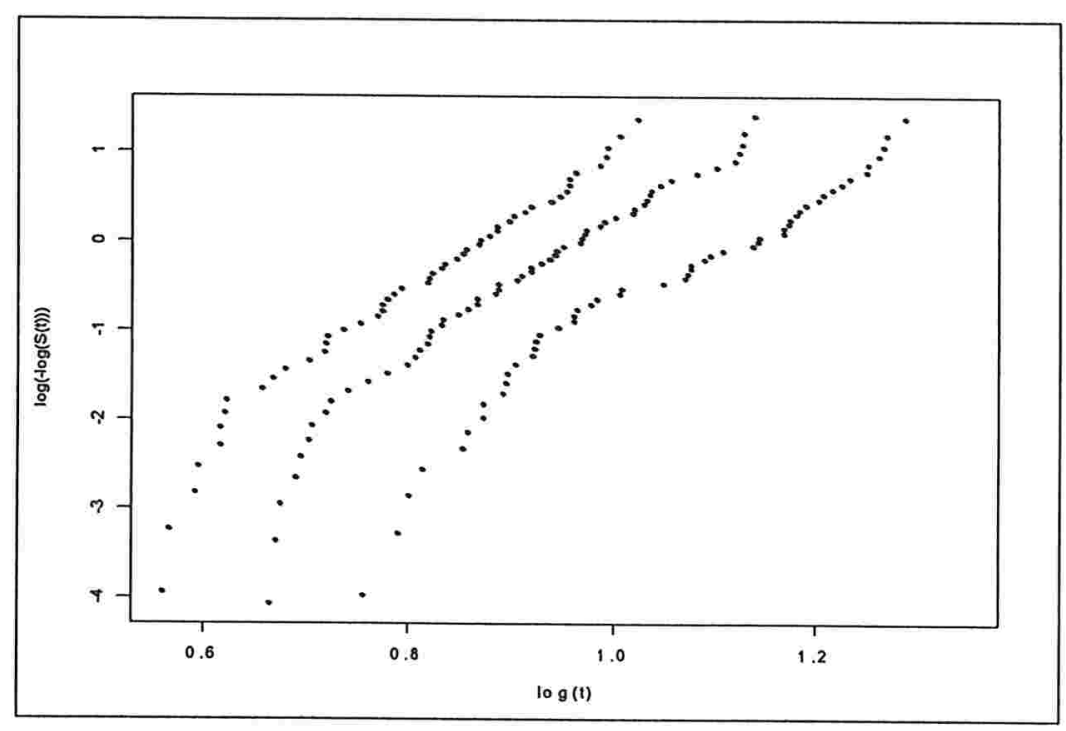

Figura 4.3: Gráfico de $\log (-\log (S(t)))$ por $\log (t)$

Risco empírico acumulado

As estimativas de máxima verossimilhança e MCMC, assim como seus intervalos de confiança e credibilidade obtidos pelo ajuste do modelo Weibull, são apresentados na Tabela (4.2). Os valores estimados para os parâmetros foram baseados em três amostras simuladas com 64000 iterações cada e um período de "burn-in" de 4000 iterações. Esse tamanho de amostra foi sugerido pelo diagnóstico de Raftery-Lewis. Para cada cadeia, foram tomados valores de $11 \mathrm{em} \mathrm{11,} \mathrm{obtendo-se} \mathrm{assim} \mathrm{uma} \mathrm{cadeia} \mathrm{de} \mathrm{tamanho} \mathrm{16364.} \mathrm{A}$ monitoração da convergência foi feita através do software CODA. Utilizando esse número de iterações, os quatro diagnósticos implementados no CODA indicaram a existência de convergência das amostras para cada um dos parâmetros. Podemos dizer que os resultados das análises clássica e Bayesiana foram parecidos, com intervalos de confiança mais fechados para a análise clássica, como era esperado. Outros valores também foram considerados para os hiperparâmetros das densidades a priori. Os resultados obtidos foram muito similares a estes, e portanto não são apresentados neste trabalho. A Figura (4.4) mostra os estresses de falha médios de $\lambda_{i}=g\left(\beta_{0}, \beta_{1}, \omega_{0}, \phi\right)$, para um comprimento i, como uma função do comprimento. O parâmetro de estresse limiar foi estimado via MCMC. Observe que o ajuste parece amplamente satisfatório. A Figura (4.5) mostra o comportamento das trajetórias das cadeias geradas e as respectivas densidades a posteriori. 
Tabela 4.2: Estimativas clássica e Bayesiana e IC de $95 \%$ para os parâmetros

\begin{tabular}{lcccc}
\hline \hline Procedimento & $\hat{\beta}_{0}$ & $\hat{\beta}_{1}$ & $\hat{\omega}_{0}$ & $\hat{\phi}$ \\
\hline EMV & 1.14 & 0.07 & 9.55 & 5.45 \\
Int. confiança & $(0.98 ; 1.29)$ & $(0.03 ; 0.14)$ & $(7.05 ; 9.97)$ & $(4.88 ; 6.01)$ \\
MCMC & 1.13 & 0.067 & 9.68 & 5.41 \\
Int. credibilidade & $(0.97 ; 1.36)$ & $(0.02 ; 0.17)$ & $(6.68 ; 9.99)$ & $(4.85 ; 6.03)$ \\
\hline \hline
\end{tabular}

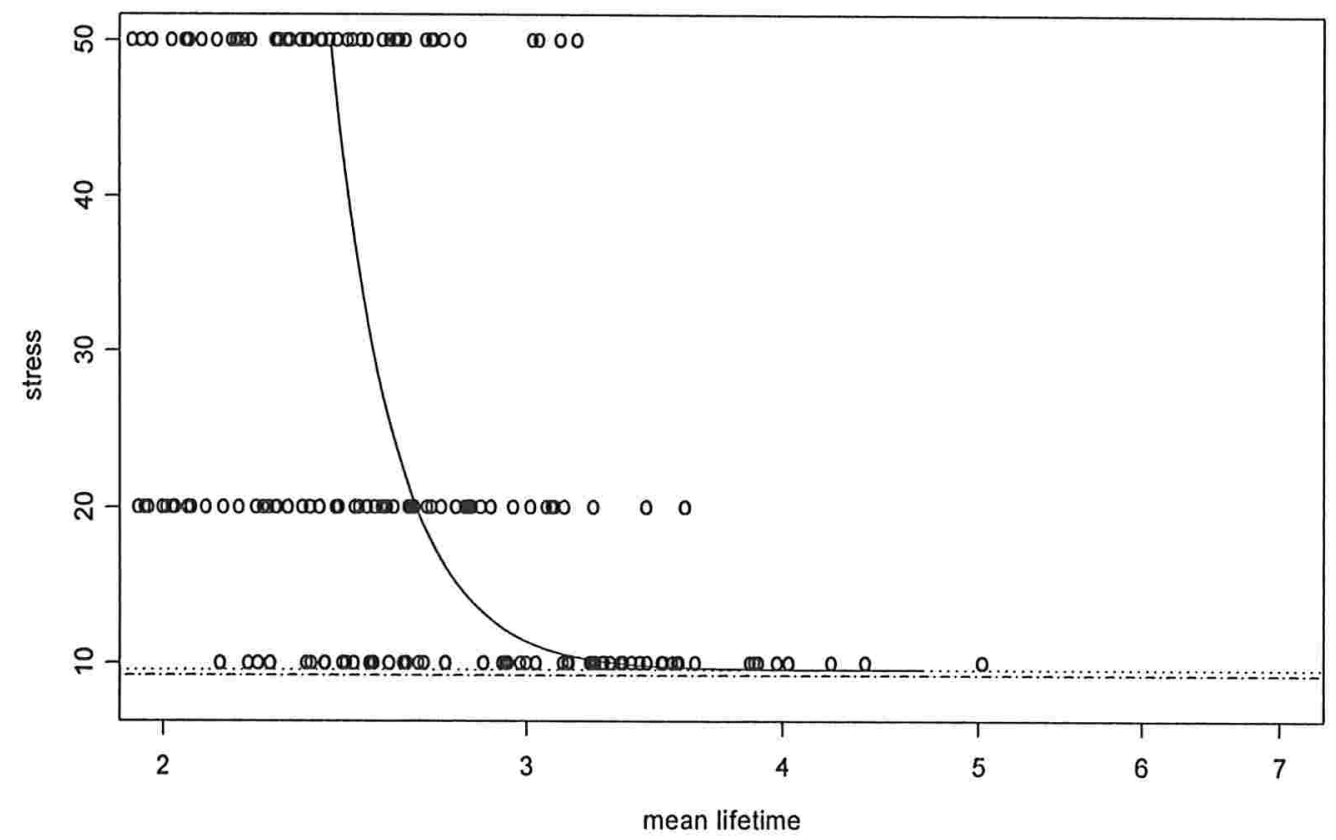

Figura 4.4: Gráfico do estresse médio ajustado como uma função do comprimento (-), com c comprimento "limiar" (- - -) e o intervalo de credibilidade de 95\% obtidos via MCMC (-.-.-) 

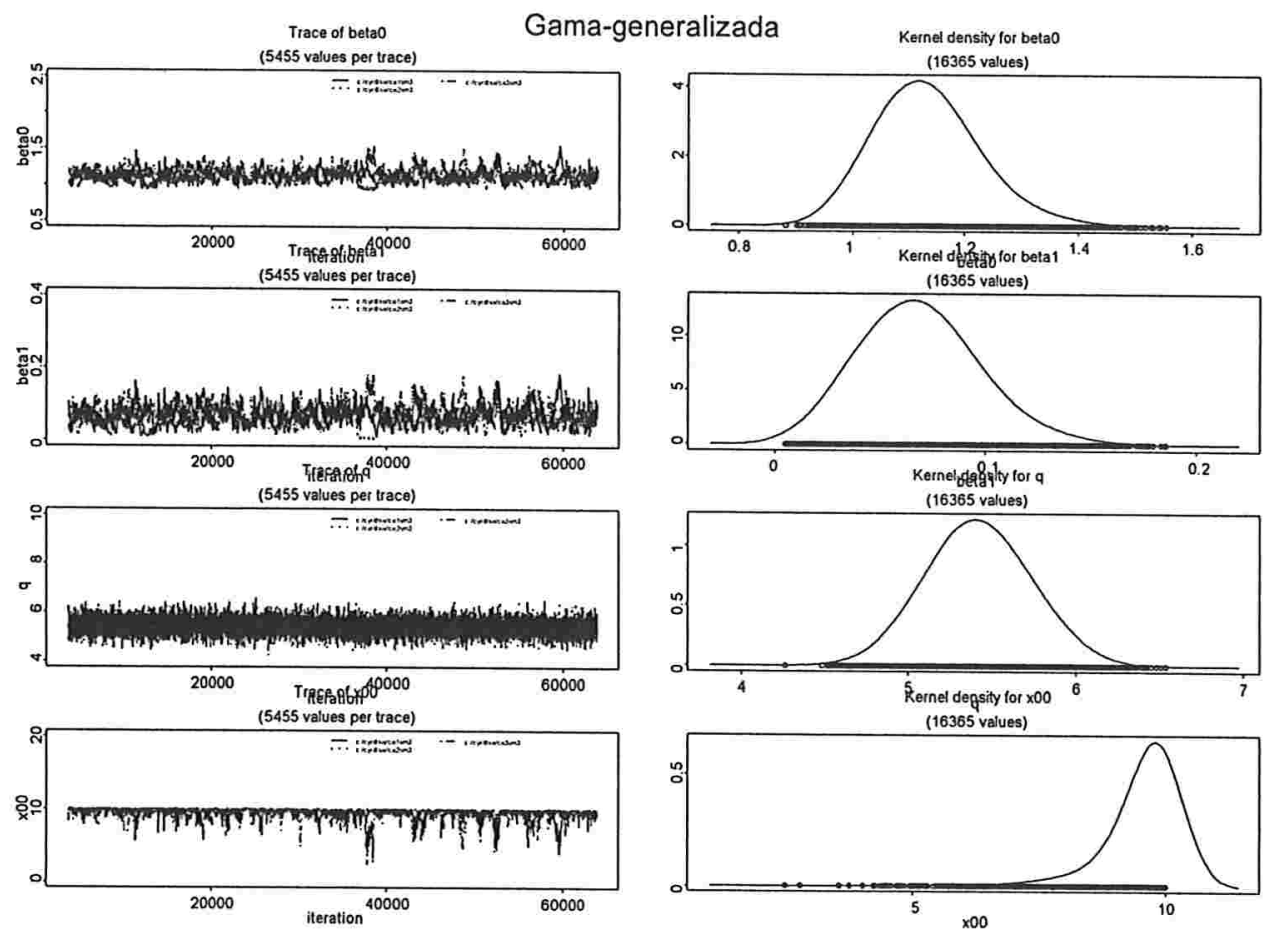

Figura 4.5: Gráficos das posterioris marginais e a história das iterações do algoritmo ARS

Com o objetivo de estudar o efeito de censuras aleatórias, nesse mesmo conjunto de dados incluímos proporções de censuras fixadas em 10\% e 40\%, respectivamente, para cada comprimento. As estimativas Bayesianas e seus intervalos de credibilidade de $95 \%$ para os parâmetros do modelo estão apresentados na Tabela 6:

Tabela 4.3: Estimativas Bayesianas e IC de $95 \%$ para os parâmetros

\begin{tabular}{lccccc}
\hline \hline Procedimento & $\hat{\beta}_{0}$ & $\hat{\beta}_{1}$ & $\omega_{0}$ & $\hat{\phi}$ & $\hat{k}$ \\
\hline MCMC 10\% & 1.18 & 0.08 & 9.61 & 5.39 & 1.06 \\
Int. credibilidade & $(0.99 ; 1.44)$ & $(0.01 ; 0.16)$ & $(3.07 ; 9.97)$ & $(4.78 ; 6.3)$ & $(0.2 ; 4.72)$ \\
MCMC 40\% & 1.24 & 0.069 & 9.59 & 5.42 & 1.49 \\
Int. credibilidade & $(1.01 ; 1.77)$ & $(-0.07 ; 0.189)$ & $(0.09 ; 10.0)$ & $(4.61 ; 6.98)$ & $(0.06 ; 5.81)$ \\
\hline \hline
\end{tabular}

\subsubsection{Dados reais 2}


Neste exemplo, consideramos os tempos de vida de fibras de carbono impregnadas com diferentes comprimentos, obtidos na "University of Surrey" por " Dr. Mark Priest" e representados na Tabela 4 de Smith (1991). Como no conjunto de dados anterior o efeito do tamanho descreve a dependência esperada da força do material em relação ao seu comprimento, assim é natural tomar o comprimento como uma covariável. Consideramos $m=4$ níveis de estresse; $x_{i}=20,50,150,300 \mathrm{~mm}$ para $i=1, \ldots, 4$, com $n_{i}=28,30,32$ e 29 observações não censuradas em cada nível. Obtemos uma estimativa $\hat{k}=1.014$ $(0.89 ; 2.43)$, o que indica que um modelo Weibull é apropriado para ajustar os dados.

Através de diagnósticos gráficos (seção 1.8), vimos que, em situações em que a variância é a mesma para todos os níveis de estresse, espera-se observar retas paralelas nos gráficos de $\log (-\log (\hat{S}(t)))$ versus $\log (t)$. Podemos observar na Figura 4.4 relações lineares porém não paralelas, o que indica que $S(t)$ é bem ajustada pelo modelo Weibull com parâmetro de forma não constante (veja Seção 1.8).

\section{Gráfico do $\ln (-\ln (K M)) v s \ln ($ tempo)}

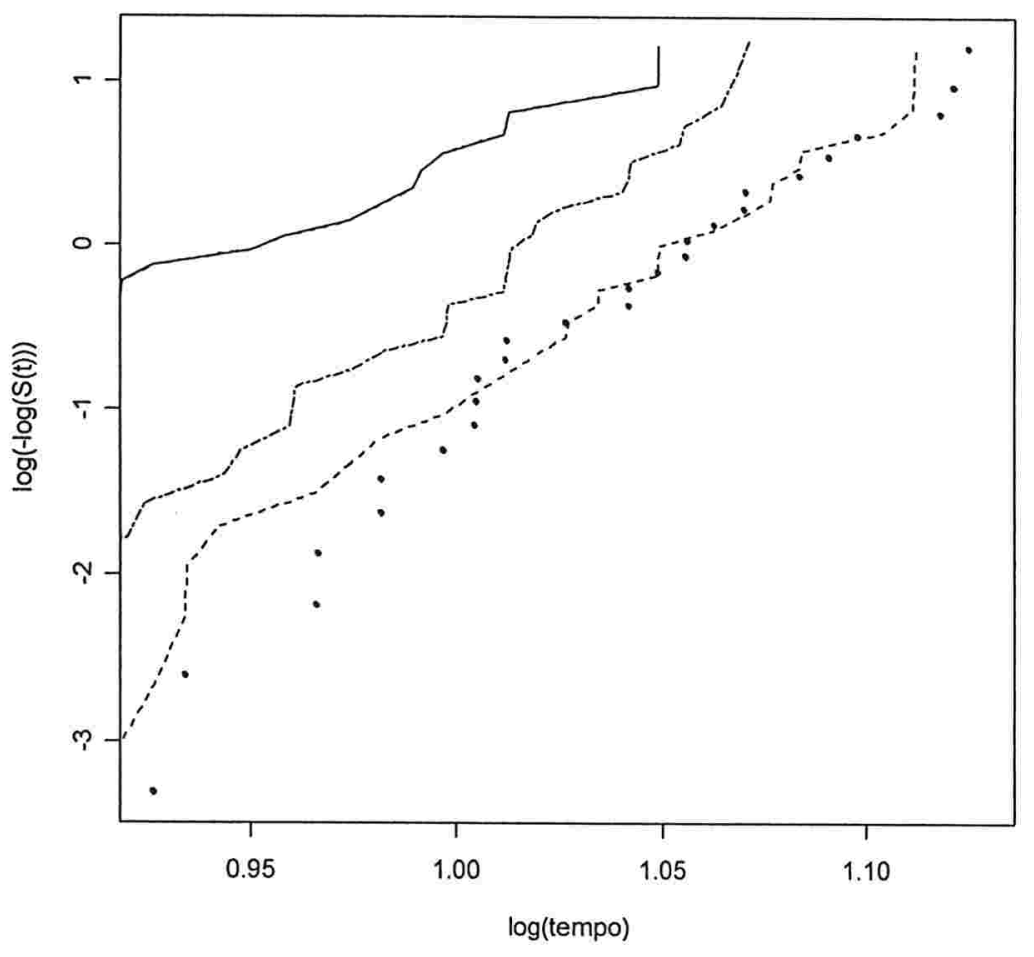

Figura 4.6: Gráfico de $\log (-\log (S(t)))$ por $\log (t)$ (...): 20mm; (---): 50mm; (-.-): 150mm; (-): 300mm

Na Tabela 4.3 são apresentadas as estimativas de máxima verossimilhança e MCMC, assim como seus intervalos de confiança e credibilidade obtidos pelo ajuste do modelo 
$\operatorname{Weibull}(\lambda, \phi)$ considerando-se $\lambda=\exp \left(-\beta_{0}+\beta_{1} \log \left(x-x_{1}+\exp (\rho)\right)\right) \mathrm{e}$ $\phi=\exp \left(-\zeta_{0}+\zeta_{1} \log \left(x-x_{1}+\exp (\rho)\right)\right)$.

Os valores estimados para os parâmetros foram baseados em três amostras simuladas com 44000 iterações cada e um período de "burn-in" de 4000 iterações. Esse tamanho de amostra foi sugerido pelo diagnóstico de Raftery-Lewis. Para cada cadeia, foram tomados valores de 13 em 13, obtendo-se assim uma cadeia de tamanho 9231. Novamente a monitoração da convergência foi feita através do software CODA. Utilizando esse número de iterações, os quatro diagnósticos implementados no CODA indicaram a existência de convergência das amostras para cada um dos parâmetros. A Figura (4.5) mostra o comportamento das trajetórias das cadeias geradas e as respectivas densidades a posteriori.

Tabela 4.4: Estimativas clássica e Bayesiana e IC de 95\% para os parâmetros

\begin{tabular}{lccccc}
\hline \hline Procedimento & $\hat{\beta}_{0}$ & $\hat{\beta}_{1}$ & $\hat{\zeta}_{0}$ & $\hat{\zeta}_{1}$ & $\hat{\omega}_{0}$ \\
\hline EMV & 1.902 & 0.241 & -6.01 & -0.63 & 19.1 \\
Int. confiança & $(1.54 ; 2.09)$ & $(0.116 ; 0.275)$ & $(-7.23 ;-3.47)$ & $(-0.38 ;-0.99)$ & $(18.01 ; 19.99)$ \\
MCMC & 1.713 & 0.1188 & -5.14 & -0.402 & 18.3 \\
Int. credibilidade & $(1.315 ; 2.16)$ & $(0.057 ; 0.185)$ & $(-8.23 ;-2.93)$ & $(-0.89 ; 0.14)$ & $(17.17 ; 19.09)$ \\
\hline \hline
\end{tabular}



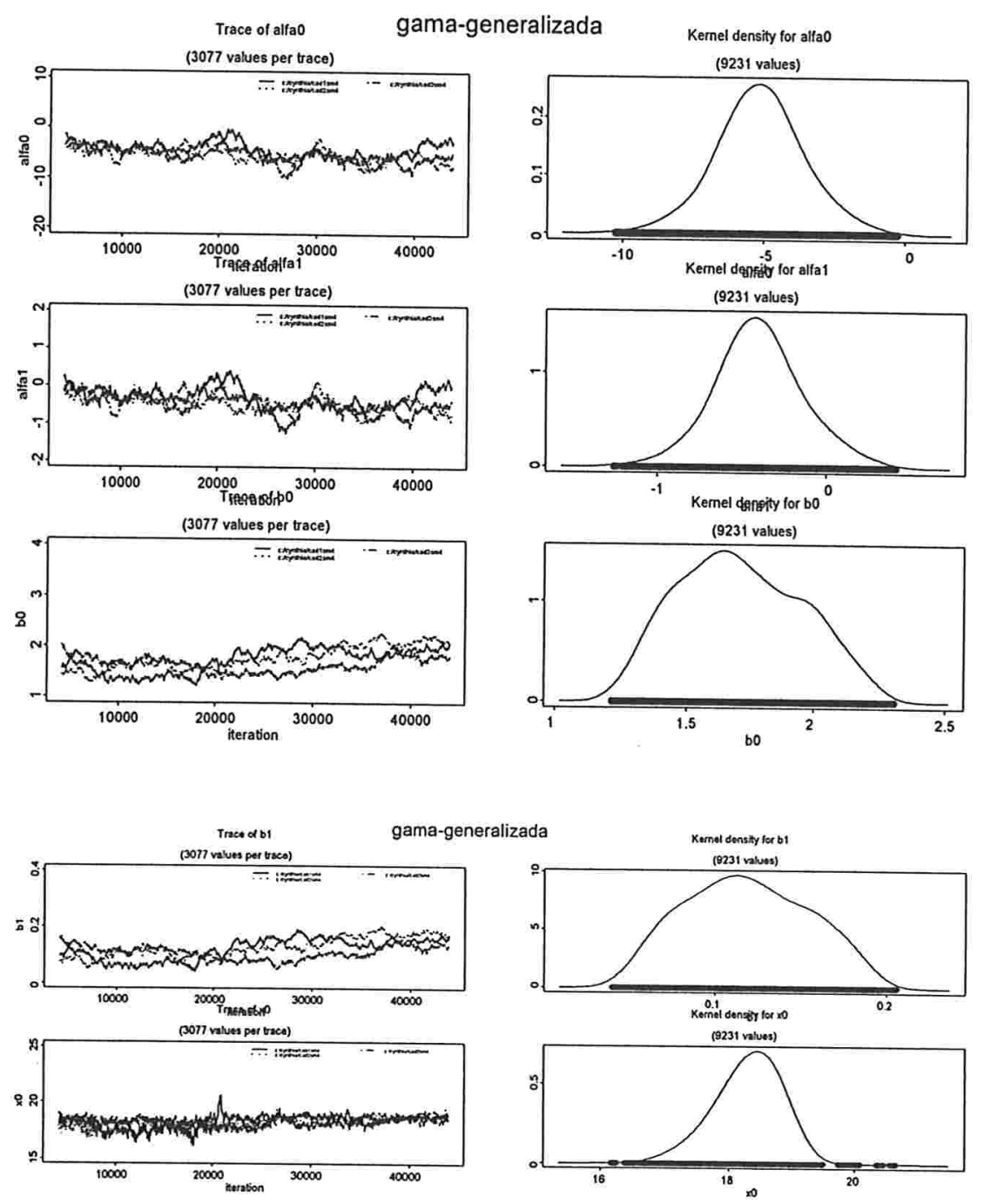

Figura 4.7: Gráficos das posterioris marginais e a história das iterações do algoritmo ARS

\subsection{Conclusões}

O modelo proposto neste capítulo é um modelo ainda mais geral de riscos híbrido com estresse limiar, o qual inclui aquele proposto no Capítulo 3. Consequentemente, tanto o modelo de riscos proporcionais de Cox $(\mathrm{PH})$, o de sobrevivência acelerado (AFT) e o modelo híbrido são casos particulares. A função de risco base no modelo foi representada através de uma abordagem paramétrica, baseada agora na distribuição gama-generalizada. Obtivemos assim uma rica classe de modelos paramétricos $\mathrm{PH} / \mathrm{AFT}$ com estresse limiar, a 
qual engloba os modelos mais importantes utilizados em confiabilidade ou análise de sobrevivência: os modelos exponencial, Weibull, gama, log-normal, qui-quadrado e ainda, com uma pequena modificação, também o modelo log-logístico. Apesar de podermos considerar toda essa família paramétrica, neste capítulo nos baseamos apenas nas distribuições gama-generalizada e log-logística, uma vez que as demais são casos particulares. Na formulação do modelo, a presença de covariáveis e observações censuradas foi considerada.

Apesar da importância do modelo gama-generalizado, ele possui o incoveniente de apresentar dificuldades operacionais em sua análise estatística. Harger e Bain (1970) mostraram que existem dificuldades de convergência para encontrar os EMV's dos parâmetros através de algoritmos numéricos, e aproximações normais podem ser muito imprecisas mesmo para grandes tamanhos de amostras. Prentice (1974) mostrou que, mesmo com o uso de reparametrizações, a estimação intervalar é imprecisa, a menos que os tamanhos de amostras sejam muito grandes. Como é usual em estudos de confiabilidade encontrar tamanhos de amostras pequenos ou moderados, uma abordagem Bayesiana do modelo foi considerada.

Exemplos em que parecia razoável permitir que a forma da distribuição dos tempos de vida fosse dependentes (e independentes) dos níveis de estresse foram apresentados.Particularmente, consideramos o modelo log-logístico em um exemplo de dados artificiais com parâmetro de forma constante e o modelo Weibull em dois exemplos reais com parâmetro de forma independente e respectivamente, dependente das covariáveis.

Para o modelo log-logístico, através de um estudo de simulação de pequena escala verificamos o comportamento da teoria assintótica para tamanhos de amostras pequenos ou moderados, e examinamos, assim, as probabilidades de cobertura dos intervalos de confiança para os parâmetros do modelo obtido. Os resultados indicaram que os procedimentos usuais de inferência clássica podem não ser muito precisos para tamanhos de amostras pequenos ou moderados. Por esse motivo, para a estimação dos parâmetros consideramos, além da abordagem clássica, uma análise Bayesiana. Tanto no caso da distribuição gama-generalizada como no da log-logística, os valores das posterioris condicionais foram amostrados através do algoritmo ARS, o qual mostrou-se novamente uma ferramenta bastante útil em problemas de estimação Bayesiana.

Através do gráfico de $\log \left(S(t)^{-1}-1\right)$ por $\log (t)$, veja Seção 1.8, observamos relações lineares e paralelas para um conjunto de dados simulados, indicando que um modelo log-logístico com parâmetro de forma constante se ajustava bem aos dados. As estimati- 
vas de máxima verossimilhança e Bayesiana dos parâmetros do modelo deram resultados similares, com intervalos de confiança maiores para a teoria assintótica, e o problema de convergência foi contornado através dos critérios de convergência implementados na biblioteca CODA. Para um conjunto de dados reais, obtivemos uma estimativa para o parâmetro de forma do modelo gama generalizado, $\hat{k}=1.05$, a qual indicou que um modelo Weibull era apropriado para ajustar os dados. Para a confirmação dessa observação, fizemos o gráfico de $\log (-\log (S(t)))$ versus $\log (t)$ e observamos relações lineares e paralelas, confirmando que $S(t)$ poderia ser realmente ajustada pelo modelo Weibull com parâmetro de forma constante.

Em muitas aplicações temos o parâmetro de forma não constante para todos os níveis de estresse. Estudos teóricos e experimentais em fadiga de materiais, por exemplo, têm mostrado que o parâmetro de forma da distribuição Weibull decresce a medida que os níveis de estresse também decrescem. Assim, consideramos um outro conjunto de dados reais onde através de técnicas gráficas, observamos relações lineares porém não paralelas, indicando que um modelo Weibull com parâmetro de forma dependente dos níveis de estresse era apropriado para ajustar os dados.

Para esses dois conjuntos de dados, utilizamos análises clássica e Bayesiana para a estimação dos parâmetros de interesse. Uma comparação entre tais análises mostrou resultados bastante próximos para todos os parâmetros e, novamente, o problema de convergência na análise Bayesiana foi contornado através do uso da biblioteca CODA.

De uma forma geral, os modelos de riscos híbridos com estresse limiar propostos nesta tese revelaram-se muito eficazes, e o objetivo de mostrar a aplicabilidade de tais modelos na presença de covariáveis e de observações censuradas foi atingido. 


\section{Capítulo 5}

\section{A Existência e importância do Estresse Limiar: Testes de Hipóteses}

Nos capítulos anteriores, desenvolvemos modelos de riscos híbridos com estresse limiar ou "threshold stress". Nossa maior atenção foi voltada a estimar este último. O conceito de estresse limiar tem sido discutido em vários campos de aplicação (Hatch, 1987), como por exemplo, em estudos de toxicologia, modelos que incluem um estresse limiar têm sido propostos (Cox, 1987). Nesses estudos, o estresse limiar pode ser considerado com o nível máximo da dose de uma certa substância tóxica, abaixo do qual podemos dizer que não existe efeito de intoxicação em um indivíduo, quando exposto a tal substância. Em aplicações industriais, o estresse limiar corresponde ao nível de estresse ( temperatura, voltagem, etc) abaixo do qual a vida de um produto pode ser considerada infinita. Entretanto, uma pergunta natural seria: o parâmetro de estresse limiar realmente existe? Para testarmos sua existência, introduzimos uma estatística de teste baseada na função escore.

Um outro ponto considerado aqui foi a importância do estresse limiar e consequências em ignorar este parâmetro quando ele existe, o que foi feito com um estudo de similação.

Este capítulo apresenta resultados baseados na teoria assintótica de primeira ordem, os quais decorrem de técnicas de linearização local baseadas nas expansões em séries de Taylor e nos teoremas centrais do limite e são válidos apenas para tamanhos de amostras $n \rightarrow \infty$. Em especial, a função escore, sendo uma soma de contribuições independentes, possui, assintoticamente, distribuição normal. Um ponto importante a ser considerado na teoria assintótica de primeira ordem é que as soluções aproximadas mostram-se, em geral, bastante razoáveis mesmo quando $n$ não é muito grande. 


\subsection{Construção da estatística de teste considerando a distribuição Weibull.}

Considerando $\omega_{0}$ o parâmetro que representa o estresse limiar e uma distribuição Weibull com parâmetros $(\phi, \lambda)$ para os tempos de sobrevivência, como no Capítulo 3, temos que a função de verossimilhança para $\lambda$ e $\phi$, com os dados sob um esquema de censura à direita (6.17), é dada por

$$
\log L(\phi, \lambda \mid X)=l(\phi, \lambda \mid X)=\sum_{i=1}^{m} \sum_{j=1}^{n_{i}} \delta_{i j}\left\{\log \phi+\phi \log \lambda_{i}+(\phi-1) \log t_{i j}\right\}-\left(\lambda_{i} t_{i j}\right)^{\phi}
$$

em que $\lambda_{i}=\exp \left(-\beta_{0}+\beta_{1} \log \left(x_{i}-\omega_{0}\right)\right), x_{i}$ são os níveis de estresse, $t_{i j}$ os tempos de falha, $m$ o número total de níveis de estresse e $n_{i}$ o número de observações em cada nível.

O interesse deste capítulo é desenvolver um procedimento de inferência para o parâmetro $\omega_{0}$. Para avaliar a existência de tal parâmetro, iremos nos basear nas hipóteses

$$
H_{0}: \omega_{0}=0 \text { vs } H_{1}: \omega_{0} \neq 0 \text {. }
$$

O teste de escore para testar as hipóteses acima é baseado na função escore (6.16)

$$
U(\theta ; X)=\frac{\partial l(\theta ; X)}{\partial \theta}
$$

em que $\theta=\left(\beta_{0}, \beta_{1}, \phi, \omega_{0}\right)$. Sob condições de regularidade apropriadas (veja, por exemplo, Serfling, 1980), o EMV $\hat{\theta}_{X}$ pode ser obtido via maximização da log-verossimilhança (5.1), o que implica na resolução da equação de estimação $U(\theta ; X)=0$. Sabemos que $\hat{\theta}_{X}$ é consistente e assintoticamente normal com vetor de médias $\theta_{0}$ e matriz de covariâncias assintóticas dada por $\left\{E\left[I\left(\theta_{0}, X\right)\right]\right\}^{-1}$, em que $I\left(\theta_{0}, X\right)$ é a matriz de informação observada (6.19) e $\theta_{0}$ o verdadeiro valor do parâmetro. Esse fato baseia-se na seguinte propriedade do escore: $E\left[U\left(\theta_{0}, X\right)\right]=0$, sendo $\theta_{0}$ o único valor de $\theta$ que anula tal esperança. Nesta tese, trabalhamos apenas com a matriz de informação observada $I\left(\theta_{0}, X\right)$, uma vez que é complicado calcular a matriz de informação esperada de Fischer $E(I(\theta, X))$ na presença de dados censurados. No entanto, sabemos que, sob certas condições de regularidade, $I\left(\theta_{0}, X\right) / n$ é um estimador consistente de $E(I(\theta, X))$.

O teste de escore para testar as hipóteses (5.2) é baseado em 


$$
U_{\omega_{0}}=\left.\frac{\partial l(\theta ; X)}{\partial \omega_{0}}\right|_{\omega_{0}=0}=\sum_{i=1}^{m} \sum_{j=1}^{n_{i}} \frac{\phi \beta_{1}}{x_{i}}\left\{-\delta_{i j}+\left[t_{i j} \exp \left(-\beta_{0}+\beta_{1} \log x_{i}\right)\right]^{\phi}\right\} .
$$

A variância assintótica de $U_{\omega_{0}}$ é encontrada subdividindo a matriz de informação observada $I\left(\theta_{0}, X\right)$ com respeito a $\omega_{0}$ e tratando $\beta_{0}, \beta_{1}$ e $\phi$ como parâmetros de ruído ou perturbação, isto é,

$$
\left(I^{\left(\omega_{0} \omega_{0}\right)}\right)^{-1}=i_{\omega_{0} \omega_{0}}-i_{\omega_{0} \varphi}^{T} i_{\varphi \varphi}^{-1} i_{\omega_{0} \varphi}
$$

em que $\varphi=\left(\beta_{0}, \beta_{1}, \phi\right), i_{\omega_{0} \omega_{0}}$ é o elemento da matriz de informação observada com respeito a $\omega_{0}$, ou seja,

$$
i_{\omega_{0} \omega_{0}}=-\left.\frac{\partial^{2} l\left(\varphi, \omega_{0} ; X\right)}{\partial \omega_{0}^{2}}\right|_{\omega_{0}=0}
$$

o vetor $i_{\omega_{0} \varphi}^{T}$ é tal que,

$$
i_{\omega_{0} \varphi}=-\left.\frac{\partial^{2} l\left(\varphi, \omega_{0} ; X\right)}{\partial \omega_{0} \partial \varphi}\right|_{\omega_{0}=0}=\left(\left.\begin{array}{r}
-\frac{\partial^{2} l\left(\varphi, \omega_{0} ; X\right)}{\partial \omega_{0} \partial \beta_{0}} \\
-\frac{\partial^{2} l\left(\varphi, \omega_{0} ; X\right)}{\partial \omega_{0} \partial \beta_{1}} \\
-\frac{\partial^{2} l\left(\varphi, \omega_{0} ; X\right)}{\partial \omega_{0} \partial \phi}
\end{array}\right|_{\omega_{0}=0}\right)
$$

e $i_{\varphi \varphi}$ é uma "submatriz " da matriz de informação observada calculada no ponto $\omega_{0}=0$ a menos das derivadas de segunda ordem do parâmetro $\omega_{0}$, ou seja,

$$
i_{\varphi \varphi}=-\left|\frac{\partial^{2} l(\varphi ; X)}{\partial \varphi^{2}}\right|_{\omega_{0}=0}=-\left(\left.\begin{array}{ccc}
\frac{\partial^{2} l(\varphi ; X)}{\partial \beta_{0}^{2}} & \frac{\partial^{2} l(\varphi ; X)}{\partial \beta_{0} \partial \beta_{1}} & \frac{\partial^{2} l(\varphi ; X)}{\partial \beta_{0} \partial \phi} \\
\frac{\partial^{2} l(\varphi ; X)}{\partial \beta_{1} \partial \beta_{0}} & \frac{\partial^{2} l(\varphi ; X)}{\partial \beta_{1}^{2}} & \frac{\partial^{2} l(\varphi ; X)}{\partial \beta_{1} \partial \phi} \\
\frac{\partial^{2} l(\varphi ; X)}{\partial \phi \partial \beta_{0}} & \frac{\partial^{2} l(\varphi ; X)}{\partial \phi \partial \beta_{1}} & \frac{\partial^{2} l(\varphi ; X)}{\partial \phi^{2}}
\end{array}\right|_{\omega_{0}=0}\right) .
$$

As primeiras e segundas derivadas de $l(\theta ; X)$ com respeito ao vetor de parâmetros $\theta=$ $\left(\beta_{0}, \beta_{1}, \phi, \omega_{0}\right)$, quando $\omega_{0}=0$, são dadas, respectivamente, por:

$$
\begin{aligned}
& \frac{\partial l(\theta ; X)}{\partial \beta_{0}}=\sum_{i=1}^{m} \sum_{j=1}^{n_{i}}-\delta_{i j} \phi+\left\{\phi\left[t_{i j} \exp (\xi)\right]^{\phi}\right\} ; \\
& \frac{\partial l(\theta ; X)}{\partial \beta_{1}}=\sum_{i=1}^{m} \sum_{j=1}^{n_{i}}-\delta_{i j} \phi\left(\log \left(x_{i}-\omega_{0}\right)\right)-\left\{\phi\left[t_{i j} \exp (\xi)\right]^{\phi}\right\} \log \left(x_{i}-\omega_{0}\right) ; \\
& \frac{\partial l(\theta ; X)}{\partial \phi}=\sum_{i=1}^{m} \sum_{j=1}^{n_{i}} \delta_{i j}\left[\frac{1}{\phi}+\xi+\log \left(t_{i j}\right)\right]-\log \left(t_{i j} \exp (\xi)\right)\left[t_{i j} \exp (\xi)\right]^{\phi} ; \\
& \text { onde } \xi=-\beta_{0}+\beta_{1} \log x_{i} .
\end{aligned}
$$




$$
\begin{aligned}
& \frac{\partial^{2} l(\theta ; X)}{\partial \beta_{0}^{2}}=\sum_{i=1}^{m} \sum_{j=1}^{n_{i}}-\phi^{2}\left\{\left[t_{i j} \exp (\xi)\right]^{\phi}\right\} ; \\
& \frac{\partial^{2} l(\theta ; X)}{\partial \beta_{1}^{2}}=\sum_{i=1}^{m} \sum_{j=1}^{n_{i}} \phi^{2}\left(\log \left(x_{i}-\omega_{0}\right)\right)^{2}\left[t_{i j} \exp (\xi)\right]^{\phi} ; \\
& \frac{\partial^{2} l(\theta ; X)}{\partial \phi^{2}}=\sum_{i=1}^{m} \sum_{j=1}^{n_{i}}-\delta_{i j}\left(\frac{1}{\phi^{2}}\right)-\left(\log \left(t_{i j} \exp (\xi)\right)\right)^{2}\left[t_{i j} \exp (\xi)\right]^{\phi} ; \\
& \frac{\partial^{2} l(\theta ; X)}{\partial \omega_{0}^{2}}=\sum_{i=1}^{m} \sum_{j=1}^{n_{i}} \phi \frac{\beta_{1}}{\left(x_{i}-\omega_{0}\right)^{2}}\left[-\delta_{i j}+\left(t_{i j} \exp (\xi)\right)^{\phi}-\phi \beta_{1}\left[t_{i j} \exp (\xi)\right]^{\phi}\right] ; \\
& \frac{\partial^{2} l(\theta ; X)}{\partial \beta_{0} \partial \beta_{1}}=\sum_{i=1}^{m} \sum_{j=1}^{n_{i}} \phi^{2} \log \left(x_{i}-\omega_{0}\right)\left[\left(t_{i j} \exp (\xi)\right)^{\phi}\right] ; \\
& \frac{\partial^{2} l(\theta ; X)}{\partial \beta_{0} \partial \phi}=\sum_{i=1}^{m} \sum_{j=1}^{n_{i}}-\delta_{i j}+\left[\left(t_{i j} \exp (\xi)\right)^{\phi}\right]\left(1+\phi \log \left(t_{i j} \exp (\xi)\right)\right) ; \\
& \frac{\partial^{2} l(\theta ; X)}{\partial \beta_{0} \partial \omega_{0}}=\sum_{i=1}^{m} \sum_{j=1}^{n_{i}}-\phi^{2} \frac{\beta_{1}}{\left(x_{i}-\omega_{0}\right)}\left[\left(t_{i j} \exp (\xi)\right)^{\phi}\right] ; \\
& \frac{\partial^{2} l(\theta ; X)}{\partial \beta_{1} \partial \phi}=\sum_{i=1}^{m} \sum_{j=1}^{n_{i}} \log \left(x_{i}-\omega_{0}\right)\left[\delta_{i j}+\left(t_{i j} \exp (\xi)\right)^{\phi}+\phi \log \left(t_{i j} \exp (\xi)\right)\left[t_{i j} \exp (\xi)\right]^{\phi}\right] ; \\
& \frac{\partial^{2} l(\theta ; X)}{\partial \omega_{0} \partial \phi}=\sum_{i=1}^{m} \sum_{j=1}^{n_{i}} \frac{\beta_{1}}{\left(x_{i}-\omega_{0}\right)}\left[-\delta_{i j}+\left(t_{i j} \exp (\xi)\right)^{\phi}+\phi \log \left(t_{i j} \exp (\xi)\right)\left[t_{i j} \exp (\xi)\right]^{\phi}\right] .
\end{aligned}
$$

A estatística de teste de escore para testar $H_{0}: \omega_{0}=0$ é, portanto, definida como

$$
S=\hat{U}_{\omega_{0}}^{\prime} \hat{I}^{\left(\omega_{0} \omega_{0}\right)} \hat{U}_{\omega_{0}}
$$

em que $\hat{U}_{\omega_{0}}=U_{\omega_{0}}(\hat{\varphi}), \quad \hat{I}^{\left(\omega_{0} \omega_{0}\right)}=I^{\left(\omega_{0} \omega_{0}\right)}(\hat{\varphi})$ e $\hat{\varphi}=\left(\hat{\beta}_{0}, \hat{\beta}_{1}, \hat{\phi}\right)$ é o estimador de máxima verossimilhança de $\varphi$ quando $\omega_{0}=0$, o qual é obtido resolvendo-se a equação $\frac{\partial l(\theta ; X)}{\partial \theta}=0$ (5.3), com $\omega_{0}=0$.

É importante lembrar que a estatística de escore baseia-se na normalidade assintótica da componente da função escore $U_{\omega_{0}}$ correspondente ao vetor de parâmetros de interesse, isto é,

$$
U_{\omega_{0}} \stackrel{D}{\longrightarrow} N\left(0,\left(I^{\left(\omega_{0} \omega_{0}\right)}\right)^{-1}\right)
$$

Assim, a distribuição assintótica da estatística $S$ dada por (5.9) segundo $H_{0}: \omega_{0}=0$ segue diretamente da convergência (5.10) e implica que

$$
S \stackrel{D \longrightarrow}{\longrightarrow} \chi_{(1)}^{2}
$$

ou seja, quando $n \rightarrow \infty, S$ converge para uma distribuição qui-quadrado com 1 grau de liberdade. 
Uma outra possibilidade para testar a hipótese $H_{0}: \omega_{0}=0$ é usar o teste da razão de verossimilhanças:

$$
T R V=2\{l(\hat{\varphi} ; X)-l(\hat{\theta} ; X)\}=2\left\{l\left(H_{0}\right)-l\left(H_{1}\right)\right\}
$$

em que $l\left(H_{0}\right)$ e $l\left(H_{1}\right)$ são as verossimilhanças calculadas sob a hipótese nula e sob a hipótese alternativa, respectivamente. Sob $H_{0}$, é possível mostrar (Wilks, 1938) que

$$
T R V \stackrel{D}{\longrightarrow} \chi_{(1)}^{2}
$$

Portanto, as estatísticas (5.9) e (5.11) são equivalentes até primeira ordem, uma vez que ambas convergem sob a hipótese nula para um distribuição $\chi_{(1)}^{2}$. A estatística de escore tem a vantagem de depender somente da EMV segundo a hipótese nula, enquanto que a estatística da razão de verossimilhanças depende também das EMV segundo a hipótese alternativa.

\subsection{Construção da estatística de teste considerando a distribuição Log-logística}

Considerando $\omega_{0}$ o parâmetro que representa o estresse limiar e uma distribuição loglogística com parâmetros $(\phi, \lambda)$ para os tempos de sobrevivência, como no Capítulo 4 , temos que a função de verossimilhança para $\lambda$ e $\phi$, com os dados sob um esquema de censura a direita (4.21), é dada por

$$
\begin{gathered}
\log L(\phi, \lambda \mid X)=l(\phi, \lambda \mid X)=\sum_{i=1}^{m} \sum_{j=1}^{n_{i}} \delta_{i j}\left\{\log \phi+\phi \log \lambda_{i}+(\phi-1) \log t_{i j}\right\}-(5.1) \log \left(1+\left(\lambda_{i} t_{i j}\right)^{\phi}\right) \\
\left(\delta_{i j}+1\right)
\end{gathered}
$$

em que $\lambda_{i}=\exp \left(-\beta_{0}+\beta_{1} \log \left(x_{i}-\omega_{0}\right)\right), x_{i}$ são os níveis de estresse, $t_{i j}$ os tempos de falha, $m$ o número total de níveis de estresse e $n_{i}$ o número de observações em cada nível.

O teste de escore para testar as hipóteses (5.2) é baseado em 
$U_{\omega_{0}}=\left.\frac{\partial l(\theta ; X)}{\partial \omega_{0}}\right|_{\omega_{0}=0}=\sum_{i=1}^{m} \sum_{j=1}^{n_{i}} \frac{\phi \beta_{1}}{x_{i}}\left\{-\delta_{i j}+\left(\delta_{i j}+1\right)\left(\frac{\left[t_{i j} \exp \left(-\beta_{0}+\beta_{1} \log x_{i}\right)\right]^{\phi}}{1+\left[t_{i j} \exp \left(-\beta_{0}+\beta_{1} \log x_{i}\right)\right]^{\phi}}\right)\right\}$.

A variância assintótica de $U_{\omega_{0}}$ é encontrada subdividindo a matriz de informação observada $I\left(\theta_{0}, X\right)$ com respeito a $\omega_{0}$ e tratando $\beta_{0}, \beta_{1}$ e $\phi$ como parâmetros de ruído ou perturbação, isto é,

$$
\left(I^{\left(\omega_{0} \omega_{0}\right)}\right)^{-1}=i_{\omega_{0} \omega_{0}}-i_{\omega_{0} \varphi}^{\prime} i_{\varphi \varphi}^{-1} i_{\omega_{0} \varphi}
$$

em que $\varphi=\left(\beta_{0}, \beta_{1}, \phi\right), i_{\omega_{0} \omega_{0}}$ é o elemento da matriz de informação observada com respeito a $\omega_{0}$ como em (5.6), ou seja,

$$
i_{\omega_{0} \omega_{0}}=-\left.\frac{\partial^{2} l\left(\varphi, \omega_{0} ; X\right)}{\partial \omega_{0}^{2}}\right|_{\omega_{0}=0}
$$

o vetor $(5.7)$

$$
i_{\omega_{0} \varphi}^{\prime}=-\frac{\partial^{2} l\left(\varphi, \omega_{0} ; X\right)}{\partial \omega \partial \varphi} \mid
$$

e $i_{\varphi \varphi}$ é uma "submatriz" da matriz de informação observada calculada no ponto $\omega_{0}=0$ a menos das derivadas de segunda ordem do parâmetro $\omega_{0}$ (5.8), ou seja,

$$
i_{\varphi \varphi}=-\left|\frac{\partial^{2} l(\varphi ; X)}{\partial \varphi^{2}}\right|_{\omega_{0}=0}
$$

Como na Seção 5.1, a estatística de teste de escore para testar $H_{0}: \omega_{0}=0$ é, portanto, definida como

$$
S=\hat{U}_{\omega_{0}}^{\prime} \hat{I}^{\left(\omega_{0} \omega_{0}\right)} \hat{U}_{\omega_{0}}
$$

em que $\hat{U}_{\omega_{0}}=U_{\omega_{0}}(\hat{\varphi}), \quad \hat{I}^{\left(\omega_{0} \omega_{0}\right)}=I^{\left(\omega_{0} \omega_{0}\right)}(\hat{\varphi})$ e $\hat{\varphi}=\left(\hat{\beta}_{0}, \hat{\beta}_{1}, \hat{\phi}\right)$ é o estimador de máxima verossimilhança de $\varphi$ quando $\omega_{0}=0$, o qual é obtido resolvendo-se a equação $\frac{\partial l(\theta ; X)}{\partial \theta}=0$ (5.13), $\operatorname{com} \omega_{0}=0$.

\subsection{Estudos de simulação}

Estudos de simulação foram realizados com o objetivo de calcular o tamanho e o poder do teste de escore para $\omega_{0}$. Os tempos de vida foram gerados primeiro a partir de uma 
distribuição Weibull com parâmetros $\phi$ e $\lambda_{i}$, onde $\lambda_{i}=\exp \left(-\beta_{0}+\beta_{1} \log \left(x_{i}-\omega_{0}\right)\right)$, em três níveis de covariáveis $x_{i}=10,20$ e 50 com 10,30,100 e 150 ítens não censurados em cada nível. Os valores dos parâmetros foram fixados em $\beta_{0}=1.63, \beta_{1}=0.2$ e $\phi=5.3$, e a partir destes valores 1000 amostras foram geradas para cada caso. Também foram gerados tempos de vida de um modelo $\log$ - $\operatorname{logístico}(\phi, \lambda)$ com $\lambda$ como acima, em três níveis de covariáveis $x_{i}=5,10,15$ com $n=10,50$ e 150 ítens não censurados em cada nível (como na seção 4.5.1). Para tal modelo os valores dos parâmetros foram fixados em $\beta_{0}=2.5$, $\beta_{1}=2$ e $\phi=3$ e utilizando esses valores 1000 amostras foram geradas para cada caso. O tamanho do teste $(\alpha)$ é baseado na hipótese nula $H_{0}: \omega_{0}=0$ e seu poder $(1-\beta)$ na alternativa $H_{1}: \omega_{0} \neq 0$. Para um nível nominal de $95 \%$, a distribuição assintótica da estatística $\chi_{(1)}^{2}$ é de 3.8415 .

Consideramos, neste capítulo, técnicas de simulação "bootstrap" paramétrico como uma alternativa aos testes de hipóteses formais baseados nas aproximações assintóticas. Assim como outros testes estatísticos, os testes "bootstrap" são baseados em uma estatística de teste $q$ que mede a discrepância entre os dados e a hipótese nula $H_{0}$. O nível de evidência contra $H_{0}$ da estatística de teste, obtido a partir do valor observado $q_{0}$, é dado pela probabilidade de significância (ou p-valor). Neste trabalho, para testar $H_{0}$ consideramos $q$ como a estatística de escore construída. Valores grandes de $q$ indicam rejeição da hipótese nula. Para a obtenção de $q$, utilizamos técnicas de reamostragem, isto é, para cada reamostra, calculamos as estatísticas $S$ e, no final de $R$ reamostragens, obtivemos as estatísticas ordenadas $q_{1}^{*}<q_{2}^{*}<\ldots<q_{R}^{*}$, o que nos permitiu então determinar a localização $q_{0}$ obtida a partir da amostra original, dentre as $q$ ordenadas, calculando o p-valor empírico, isto é, $\hat{p}=\frac{1}{R} \sum_{i=1}^{R} I\left(q_{i}^{*} \geq q_{0}\right)$, em que $I($.$) é a função indicadora. Rejeita-se a$ hipótese nula se $\hat{p} \leq \alpha^{*}$, em que $\alpha^{*}$ é o nível de significância do teste.

Por meio de $R=999$ simulações "bootstrap" paramétrico, obtivemos o tamanho e o poder dos testes de escore dados nas Tabelas 5.1 e 5.2, respectivamente, para o modelo Weibull e nas Tabelas 5.3 e 5.4 para a distribuição log-logística. Podemos observar nas Tabelas 5.1 e 5.3 que o erro tipo I de falsamente detectar um estresse limiar foi de aproximadamente $5 \%$ apenas para amostras grandes $(n \geq 100)$ e, nas Tabelas 5.2 e 5.4 que o poder para detectar um estresse limiar significante foi próximo de $100 \%$ para tamanho de amostra grande. 
Tabela 5.1: Tamanho do teste de Score para $H_{0}: \omega_{0}=0$

\begin{tabular}{ccccc}
\multicolumn{4}{c}{ Modelo Weibull } \\
\hline \hline$n$ & 10 & 30 & 100 & 150 \\
\hline$\alpha$ & 0.12 & 0.084 & 0.063 & 0.048 \\
\hline \hline
\end{tabular}

Tabela 5.2: Poder do teste de Score para $n=150$

Modelo Weibull

\begin{tabular}{lcccc}
\hline \hline$\omega_{0}$ & 9.0 & 9.5 & 9.8 & 0.0 \\
\hline \hline$(1-\beta)$ & 0.998 & 1.000 & 1.000 & $\ldots-$ \\
\hline \hline
\end{tabular}

Tabela 5.3: Tamanho do teste de Score para $H_{0}: \omega_{0}=0$

\begin{tabular}{cccc}
\multicolumn{4}{c}{ Modelo Log-logístico } \\
\hline \hline$n$ & 10 & 50 & 150 \\
\hline$\alpha$ & 0.14 & 0.071 & 0.052 \\
\hline
\end{tabular}

Tabela 5.4: Poder do teste de Score para $n=150$ Modelo Log-logístico

\begin{tabular}{lcccc}
\hline \hline$\omega_{0}$ & 4.0 & 4.5 & 4.8 & 0.0 \\
\hline \hline$(1-\beta)$ & 0.987 & 1.000 & 1.000 & $\ldots--$ \\
\hline \hline
\end{tabular}

\subsection{Aplicação a um conjunto de dados reais}

Consideremos o conjunto de dados da Tabela 3 de Smith (1991), como no Capítulo 4, para $m=3$ níveis diferentes com $n_{i}=63,70,62$ observações não censuradas em cada nível. Para esse conjunto de dados, vimos que o modelo Weibull com parâmetro de forma constante ajustou perfeitamente os dados (veja seção 4.6.2). As estimativas de máxima verossimilhança para os parâmetros de ruído foram calculadas sob $H_{0}: \omega_{0}=0$ usando a rotina nlm do pacote $\mathrm{R}$, a qual encontra o máximo local usando um método de quasi Newton-Rhapson. As estimativas obtidas foram $\hat{\beta}_{0}=1.63, \hat{\beta}_{1}=0.20$ e $\hat{\phi}=5.29$, com intervalos de $95 \%$ de confiança assintóticos iguais a $(1.51 ; 1.75),(0.16 ; 0.24)$ e $(4.74 ; 5.83)$, respectivamente.

A estatística de teste de escore (5.9) foi dada por $63.24(p-$ valor $<0.0001)$, a qual é maior que $\chi_{(0.05)}^{2}(1)$. O valor da estatística do teste da razão de verossimilhanças também foi calculado, uma vez que obtivemos as emv dos parâmetros $\omega_{0}, \beta_{0}, \beta_{1}$ e $\phi$ no Capítulo 4. Os valores de $l\left(H_{0}\right)$ e $l\left(H_{1}\right)$ foram 151.82 e 136.93 , respectivamente, fornecendo um valor para a estatística (5.11) $T R V=29.78$ ( $p$-valor < 0.0001). Para ambos os testes, 
o $p$-valor indicado leva à rejeição da hipótese nula $H_{0}: \omega_{0}=0$. O teste de escore tem, assintoticamente, as mesmas propriedades que o teste da razão de verossimilhanças, mas o valor do teste de escore foi bem maior que o TRV. Esses resultados indicam que existe um nível potencial de estresse limiar.

\subsection{A importância do estresse limiar.}

Nesta Seção realizamos um estudo de simulação com o objetivo de mostrar a importância do estresse limiar, quando este existe, e suas consequências em ignorá-lo.

Os tempos de vida foram simulados, a partir de uma distribuição Weibull $(\lambda, \phi)$ onde $\lambda$ é dado pelo modelo de lei da potência inversa (como na Seção 5.3), com parâmetro de estresse limiar $\omega_{0}=4.8, \beta_{0}=2, \beta_{1}=2$ e $\phi=3$ em três níveis de covariáveis $x_{i}=5,10$ e $15 \operatorname{com} n=50$ ítens não censurados em cada nível.

A Tabela 5.5 e 5.6 apresenta as estimativas de máxima verossimilhança (juntamente com seus erros quadráticos médios) e seus respectivos intervalos de $95 \%$ de confiança para todos os parâmetros do modelo, considerando-se $\lambda=\exp \left(-\beta_{0}+\beta_{1} \log \left(x_{i}-\omega_{0}\right)\right)$ (Modelo I) e $\lambda=\exp \left(-\beta_{0}+\beta_{1} \log \left(x_{i}\right)\right.$ ) (Modelo II), respectivamente.

Observe que o erro quadrático médio para o modelo II é bem maior que do modelo I, indicando assim uma forte evidência em favor do modelo completo (I). Ou seja, ignorar o efeito do estresse limiar quando este existe, nos leva a uma superestimação dos parâmetros.

Tabela 5.5: Estimativas clássica e IC de 95\% para os parâmetros

\begin{tabular}{lcccc}
\hline \hline Procedimento & $\hat{\beta}_{0}$ & $\hat{\beta}_{1}$ & $\widehat{\phi}$ & $\hat{\omega}_{0}$ \\
\hline EMV & 1.76 & 1.91 & 2.83 & 4.81 \\
& $(E Q M=0.057)$ & $(E Q M=0.008)$ & $(E Q M=0.030)$ & $(E Q M=0.001)$ \\
Int. confiança & $(1.424 ; 2.106)$ & $(1.745 ; 2.088)$ & $(2.528 ; 3.068)$ & $(4.741 ; 4.893)$ \\
\hline \hline
\end{tabular}

Tabela 5.6: Estimativas clássica e IC de 95\% para os parâmetros

\begin{tabular}{lccc}
\hline \hline PProcedimento & $\hat{\beta}_{0}$ & $\hat{\beta}_{1}$ & $\widehat{\phi}$ \\
\hline EMV & 16.03 & 7.02 & 1.52 \\
& $(E Q M=196.1)$ & $(E Q M=25.20)$ & $(E Q M=2.176)$ \\
Int. confiança & $(15.688 ; 16.319)$ & $(6.889 ; 7.167)$ & $(1.385 ; 1.664)$ \\
\hline \hline
\end{tabular}




\subsection{Conclusões}

Neste capítulo estudamos a existência do parâmetro de estresse limiar ou "threshold", através de uma estatística de teste de Escore construída e por meio de testes da razão de verossimilhanças. A construção da estatística de teste foi feita considerando as distribuições Weibull e log-logística. Para o modelo com estresse limiar, os demais parâmetros foram tratados como parâmetros de ruído, embora eles sejam necessários no modelo. O principal objetivo foi detectar um parâmetro de estresse limiar significativo, pois sua existência é de fundamental importância para usuários de testes de sobrevivência acelerados. Simulações considerando ambos os modelos foram realizadas com o objetivo de calcular o tamanho e o poder do teste de escore para o parâmetro de estresse limiar. Como alternativa aos testes de hipóteses formais baseados em aproximações assintóticas, utilizamos técnicas de simulação "bootstrap" paramétrico. Nossos resultados mostraram, para ambas as simulações, que o erro tipo I de falsamente detectar um estresse limiar foi de apenas $5 \%$ aproximadamente para amostras grandes $(n \geq 100)$, e que o poder para detectar um estresse limiar significativo foi próximo de $100 \%$ para tamanhos de amostras grandes.

Fizemos uso do teste de Escore e razão de verossimilhanças para analisar um conjunto de dados reais. Os resultados obtidos indicaram um nível potencial de estresse limiar.

Um estudo de simulação foi realizado com o objetivo de mostrar a importância de considerar o parâmetro de estresse limiar, quando este existe. Consideramos o modelo completo (com parâmetro de estresse limiar) e o modelo sem o parâmetro de estresse limiar. Para efeito de comparação entre os dois modelos e erro quadrático médio foi calculado e este apresentou resultados bem maiores para o modelo incompleto. 


\section{Capítulo 6}

\section{Modelos de Múltiplos Riscos}

\subsection{Introdução}

Os modelos de múltiplos riscos, como o Weibull múltiplo (Louzada-Neto, 1999), são modelos alternativos importantes que podem ser utilizados nos mais variados problemas de modelagem de dados de sobrevivência e confiabilidade. Em geral, esses modelos são aplicados em problemas específicos como os de riscos competitivos, sistemas mascarados e riscos complementares, nos quais existe informação total ou parcial quanto às causas responsáveis pelas falhas (ver por exemplo, David e Moeschberger, 1978 e Basu e Klein, 1982).

Em comparação com os modelos tradicionalmente utilizados na modelagem de dados que representam o tempo até a ocorrência de determinado evento de interesse, morte ou falha, como os modelos Weibull, log-logístico e gama-generalizado, os de múltiplos riscos têm a característica de permitir o ajuste de formas de riscos não monótonas, tais como em forma de " $U$ " e também as multimodais, possibilitando assim uma maior flexibilidade na análise de dados de sobrevivência.

Em análise de sobrevivência ou confiabilidade, são comuns as situações em que um indivíduo está sujeito a $r \geq 2$ causas de falhas, ou em que um sistema é composto por $k$ diferentes elementos e a verdadeira causa de falha é parcial ou completamente desconhecida. Supondo que $X_{r}, r=1, \ldots, p$ represente o tempo até a falha devida à $r$-ésima causa, com os $X_{r}$ independentes, tem-se que a variável aleatória observada, no contexto de riscos competitivos ou múltiplos riscos, é dada por $T=\min \left(X_{1}, \ldots, X_{r}\right)$ (Nelson, 1982; Lawless, 1982). Dessa forma, sob a suposição de que as causas de falha são independentes, a função de risco global é dada por 


$$
h(t)=\sum_{r=1}^{p} h_{j}(t),
$$

onde $h_{r}(t)$ é a função de risco associada à $r$ - ésima causa.

Uma possível parametrização de (6.1), proposta por Louzada-Neto (1999) e conhecida como modelo de múltiplos riscos, é dada por

$$
h_{r}(t)=\frac{\phi_{r} t^{\phi_{r}-1}}{\mu_{r}^{\phi_{r}}} q_{r}\left(t ; \phi_{r}, \lambda_{r}, k\right),
$$

onde $q_{r}($.$) são funções paramétricas positivas para r=1, \ldots, p$, iguais a 1 quando seus argumentos valem zero, e $\phi, \mu$ e $k$ são parâmetros positivos desconhecidos.

Considerando em (6.2) que

$$
q_{r}\left(t ; \phi_{j}, \lambda_{j}, k\right)=\frac{\Gamma(k)^{-1} u^{k-1} e^{-u}}{1-I(k ; u)},
$$

onde $u=(\lambda t)^{\phi}$ e $I(k ; u)=\Gamma(k)^{-1} \int_{0}^{u} a^{k-1} e^{-a} d a$ é uma função gama incompleta (Lawless, 1982), temos em (6.1) a função de risco global do modelo gama-generalizado múltiplo (Lawless, 1982). Casos especiais são dados pelos modelos Weibull múltiplo $(k=1)$ e log-normal múltiplo $(k \longrightarrow \infty)$.

Para uma interpretação direta de (6.1), considere um sistema ligado em série (veja, por exemplo, Nelson, 1982), no qual cada componente falha de forma independente. Uma vez que esse sistema só funciona se todos os sub-sistemas funcionarem, tem-se que o risco global é dado pela soma dos riscos individuais. Em termos da função de sobrevivência, a confiabilidade do sistema é dada pelo produto das confiabilidades de cada sistema, ou seja, $S(t)=\prod_{r=1}^{p} S_{j}(t)$. Por outro lado, no contexto de riscos complementares (Basu e Klein, 1982), é possível pensar em um sistema ligado em paralelo e, neste caso, a variável aleatória observada $T$ é dada por $T=\max \left(X_{1}, \ldots, X_{p}\right)$, o que corresponde ao fato de que o sistema funciona se pelo menos um dos seus sub-sistemas funcionar.

Em muitas situações, a suposição de independência entre as diferentes causas de falhas não é razoável (Gelfand et al., 2000), uma vez que tais causas podem agir até a falha simultaneamente ao longo do tempo. Nesses casos, o tempo até a falha acarretada por uma causa específica pode estar correlacionado com o tempo até a falha por outras causas. Por outro lado, mesmo sob a suposição de independência entre as causas de falhas, o modelo (6.1) pode ser considerado em muitas situações práticas em que existem, por exemplo, falhas por causas iniciais (infant mortality) e falhas pelo desgaste (wearout mortality) 
de determinado equipamento (Meeker e Escobar, 1998; Nelson, 1990). Diante disso, é razoável admitir a independência entre ambos os tipos de falhas (Berger e Sun, 1993). Geralmente, nos casos de infant mortality e wearout mortality, a função de risco tem a forma de um " $U$ ".

Considerando a função de risco global (6.1), a função densidade de probabilidade da variável aleatória $T=\min \left(X_{1}, \ldots, X_{p}\right)$ pode ser escrita na forma

$$
f(t)=\sum_{r=1}^{p} h_{r}(t) \prod_{r=1}^{p} S_{j}(t)=\sum_{r=1}^{p} h_{j}(t) \prod_{l=1, l r}^{p} S_{r}(t) .
$$

Note que, em (6.4), utiliza-se o fato de que $f(t)=h(t) S(t)$ (veja, por exemplo, Lawless, 1982). É possível observar em (6.4) que, quando uma causa específica acarreta a falha, os tempos de sobrevivência relacionados com outras causas específicas são vistos como observações censuradas.

Os modelos de múltiplos riscos são aplicados naturalmente no contexto de riscos competitivos, em que, além da variável aleatória $T$ e um possível indicador de censura, observa-se também outra variável índice que identifica a causa exata de falha.

Existem situações em que não existe informação direta a respeito da verdadeira causa de falha (Davison e Louzada-Neto, 2000; Louzada-Neto, 1999; Berger e Sun, 1996). Nesses casos, a identificação da possibilidade do ajuste de um modelo de múltiplos riscos é conduzida, geralmente, com base em diagnósticos gráficos. Uma possibilidade é considerar os gráficos conhecidos como TTT-"plots" (Mudholkar et al, 1996; Aarset, 1987).

\subsection{Determinação empírica da forma da função de risco}

A modelagem de dados de sobrevivência está vinculada à forma da função de risco e, como existem vários modelos concorrentes para modelar tais dados, é necessária a utilização de alguma metodologia para selecionar o modelo mais apropriado, antes mesmo de qualquer ajuste. Gráficos conhecidos como risco acumulado e TTT "plot" são de grande utilidade nesse contexto, pois eles determinam a forma da função de risco.

O gráfico empírico TTT (Aarset, 1987) é obtido representando

$$
G(r / n)=\left[\left(\sum_{i=1}^{r} T_{i: n}\right)+(n-r) T_{r: n}\right] /\left(\sum_{i=1}^{n} T_{i: n}\right)
$$


em que $r=1, \ldots, n$ e $T_{i: n}, i=1, \ldots, n$ são as estatísticas de ordem da amostra versus $r / n$ (Mudholkar, Srivastava, e Kolia, 1996). Curvas convexas indicam que a função de risco é decrescente, curvas côncavas correspondem a funções de risco crescentes. Caso a curva seja convexa e então côncava, a função de risco tem a forma de um " $U$ ". Várias regiões côncavas e convexas indicam o ajuste através de distribuições de múltiplos riscos (Louzada-Neto, 1999).

\subsection{Modelos de múltiplos riscos na presença de cova- riáveis}

Uma situação bastante comum na modelagem de dados de sobrevivência é aquela em que o tempo até a falha depende de covariáveis. Assim, é importante considerar generalizações de (6.1), de modo que o possível efeito de covariáveis possa ser avaliado. Uma possível generalização de (6.1) é

$$
h_{j}(t)=\frac{\phi_{r} t^{\phi_{r}-1}}{\mu_{r}^{\phi_{r}}} q_{r}\left(t ; \phi_{r}, \mu_{r}, k\right) g(z) .
$$

Em análise de sobrevivência, as covariáveis podem ser introduzidas no modelo por meio de diferentes formas funcionais. Entretanto, a mais comumente utilizada é dada por (veja, por exemplo, Lawless, 1982)

$$
g(z)=e^{z^{t} \beta_{r}}
$$

em que $\beta_{r}$ representa o vetor de parâmetros de regressão e $z$ o vetor de variáveis regressoras.

\subsection{O caso Weibull perturbado por uma exponencial}

Considerando $k=1$ em (6.3), obtemos a função de risco de uma Weibull dupla para $t$ a partir de (6.5), a qual é representada por

$$
h(t)=\sum_{r=1}^{2} \frac{\phi_{r} t^{\phi_{r}-1}}{\mu_{r}^{\phi_{r}}} g(z),
$$

em que $\mu_{j}>0$ são os parâmetros de escala e $\phi_{j}>0$ os parâmetros de forma para $j=1,2$. As distribuições de múltiplos riscos são bastante flexíveis para ajustar dados de tempo de 
vida, uma vez que a função de risco (6.6) acomoda uma ampla classe de formas de risco. Caso $\min \left(\phi_{1}, \phi_{2}\right)>1$, temos um risco crescente, enquanto que o risco é decrescente se $\max \left(\phi_{1}, \phi_{2}\right)<1$. Caso $\phi_{1}<1$ e $\phi_{2}$. $\geq 1$, então o risco tem a forma de um $U$ (Berger and Sum, 1993).

Supondo $j=2$ em (6.6), $\phi_{2} .=1, \phi_{1}=\phi, \mu_{1}=\mu_{2}=\mu$ e $g(z)=\exp \left(\beta_{1} \log \left(x_{i}-\omega_{0}\right)\right)$, temos o que chamamos uma função de risco Weibull perturbada por uma exponencial. Neste caso, a partir de (6.6) obtemos

$$
h(t)=\left(\frac{\phi t^{\phi-1}}{\mu^{\phi}}+\frac{1}{\mu}\right) \exp \left(\beta_{1} \log \left(x_{i}-\omega_{0}\right)\right) .
$$

\subsubsection{Procedimentos de máxima verossimilhança}

De acordo com a função de risco dada em (6.7) e a reparametrização $\mu=\exp \left(\beta_{0}\right)$ no parâmetro de escala, a função de log-verossimilhança é dada por

$$
\begin{aligned}
l= & \log L\left(\beta_{0}, \beta_{1}, \omega_{0}, \phi\right) \alpha \log \left(\prod_{i=1}^{n} \prod_{j=1}^{m} h\left(t_{i j}\right)^{\delta_{i j}} S\left(t_{i j}\right)\right)=\sum_{i=1}^{n} \sum_{j=1}^{m} \delta_{i j} \log \left(h\left(t_{i j}\right)\right)+\log \left(S\left(t_{i j}\right)\right)= \\
= & \sum_{i=1}^{n} \sum_{j=1}^{m}\left\{\delta_{i j}\left(\log \left(\frac{\phi t_{i j}^{\phi-1}}{\left(e^{\beta_{0}}\right)^{\phi}}+\frac{1}{e^{\beta_{0}}}\right)+\left(\beta_{1} \log \left(x_{i}-\omega_{0}\right)\right)\right)-\right. \\
& \left.\left(\left(\frac{t_{i j}}{e^{\beta_{0}}}\right)^{\phi}+\frac{t_{i j}}{e^{\beta_{0}}}\right) \exp \left(\beta_{1} \log \left(x_{i}-\omega_{0}\right)\right)\right\} .
\end{aligned}
$$

Como nos capítulos anteriores, as estimativas do vetor de parâmetros $\left(\beta_{0}, \beta_{1}, \omega_{0}, \phi\right)$ em (6.8) podem ser obtidas por meio da maximização direta da função log-verossimilhança via métodos de resolução de sistemas não lineares, enquanto que inferências podem ser conduzidas com base na normalidade assintótica dos estimadores de máxima verossimilhança (Cox e Hinkley, 1974), como descrito no Apêndice A.

\subsubsection{Estimação Bayesiana}

Para evitar instabilidade numérica, consideramos a mesma reparametrização dos capítulos anteriores, sugerida por Davison e Hinkley (1997), ou seja, $\omega_{0}=x_{1}-\exp (\rho)$. Se tomarmos as distribuições a priori da forma

$$
\left(\beta_{0}, \beta_{1}, \rho\right) \sim N\left(\mu_{i}, \sigma_{i}^{2}\right) \text { e } \phi \sim \operatorname{gama}(a, b)
$$


em que $\mu_{i}, \sigma_{i}^{2}, a, b$ são os hiperparâmetros conhecidos, as posterioris condicionais são dadas por

$$
\begin{gathered}
\pi\left(\beta_{0} \mid \beta_{1}, \rho, \phi\right) \alpha \exp \left\{\sum_{i=1}^{m} \sum_{j=1}^{n_{i}} \delta_{i j} \log \left(\frac{\phi t_{i j}^{\phi-1}}{\left(e^{\beta_{0}}\right)^{\phi}}+\frac{1}{e^{\beta_{0}}}\right)-\left(\frac{t_{i j}}{e^{\beta_{0}}}\right)^{\phi+1}\right. \\
\left.\exp \left(\beta_{1} \log \left(x_{i}-x_{1}+\exp (\rho)\right)\right)-\frac{\beta_{0}^{2}}{2 \sigma_{1}^{2}}\right\} \\
\pi\left(\beta_{1} \mid \beta_{0}, \rho, \phi\right) \alpha \exp \left\{\sum_{i=1}^{m} \sum_{j=1}^{n_{i}} \delta_{i j} \beta_{1} \log \left(x_{i}-x_{1}+\exp (\rho)\right)-\left(\frac{t_{i j}}{e^{\beta_{0}}}\right)^{\phi+1}\right. \\
\left.\exp \left(\beta_{1} \log \left(x_{i}-x_{1}+\exp (\rho)\right)\right)-\frac{\beta_{1}^{2}}{2 \sigma_{2}^{2}}\right\} \\
\pi\left(\rho \mid \beta_{0}, \beta_{1}, \phi\right) \alpha \exp \left\{\sum_{i=1}^{m} \sum_{j=1}^{n_{i}} \delta_{i j} \beta_{1} \log \left(x_{i}-x_{1}+\exp (\rho)\right)-\left(\frac{t_{i j}}{e^{\beta_{0}}}\right)^{\phi+1}\right. \\
\left.\exp \left(\beta_{1} \log \left(x_{i}-x_{1}+\exp (\rho)\right)\right)-\frac{\rho^{2}}{2 \sigma_{3}^{2}}\right\} \\
\left.\left.\pi\left(\phi \mid \beta_{0}, \beta_{1}, \rho\right) \alpha \log \left(x_{i}-x_{1}+\exp (\rho)\right)\right)-b \phi+(a-1) \log \phi\right\} \\
\left\{\sum_{i=1}^{m} \sum_{j=1}^{n_{i}} \delta_{i j} \log \left(\frac{\phi t_{i j}^{\phi-1}}{\left(e^{\beta_{0}}\right)^{\phi}}+\frac{1}{e^{\beta_{0}}}\right)-\left(\frac{t_{i j}}{e^{\beta_{0}}}\right)^{\phi+1}\right.
\end{gathered}
$$

Podemos observar que as posterioris condicionais definidas em (6.9) a (6.12) não pertencem a nenhuma família paramétrica de distribuições conhecidas. Utilizaremos assim o algoritmo ARS definido no Apêndice B.

\subsection{Exemplo: dados simulados}

Neste exemplo, os tempos foram simulados a partir de (6.7) considerando-se $\phi_{1}=0.4$, $\phi_{2}=1, \beta_{0}=2, \beta_{1}=0.8, \rho=-0.65$ e três níveis de voltagem $X_{i}=7,15,20$, com 100 observações em cada nível. A Figura (6.1) apresenta o gráficos do risco acumulado, TTT"plot" e as curvas de sobrevivência obtidas via Kaplan-Meier por meio do ajuste obtido a 
partir dos modelos Weibull perturbado por uma exponencial e Weibull simples. Observase na Figura 6.1-a uma certa curvatura, indicando que o modelo Weibull não ajusta bem os dados (no capítulo 1 vimos que $S(t)$ é bem ajustada por um modelo Weibull quando observamos relações lineares no gráfico de $\log (-\log S(t))$ versus $\log t)$, e em 6.1-b uma região convexa e então côncava quase constante, a qual indica a presença de mais de um risco. A Figura 6.1-c mostra um ajuste perfeito para o modelo Weibull perturbado por uma exponencial, e em 6.1-d podemos observar que, se for ignorada a presença de mais de um risco, o modelo Weibull simples não fornece um bom ajuste.

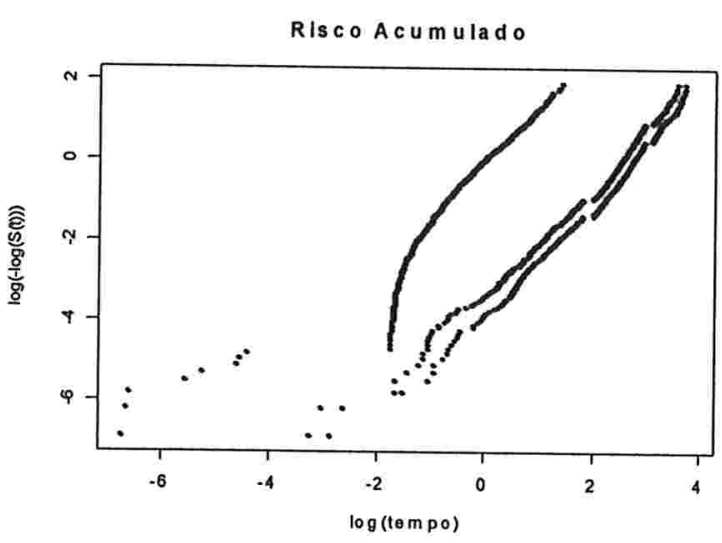

(a)

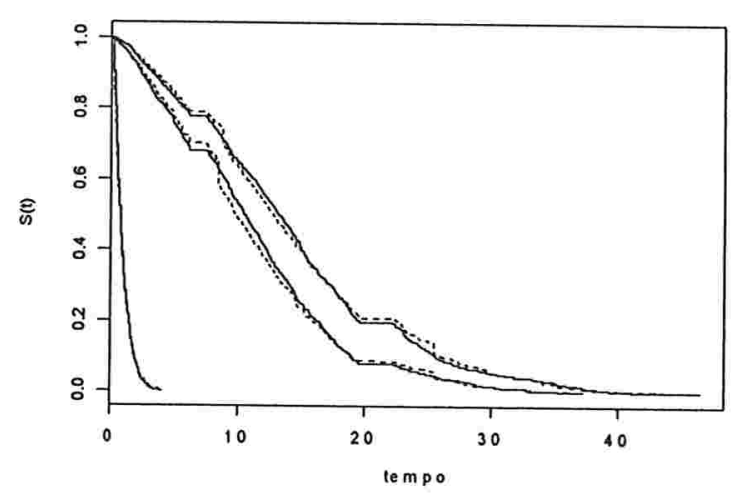

(c)

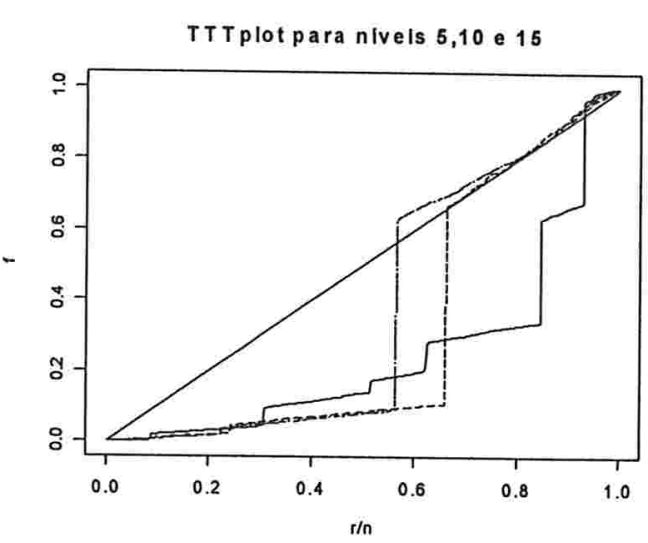

(b)

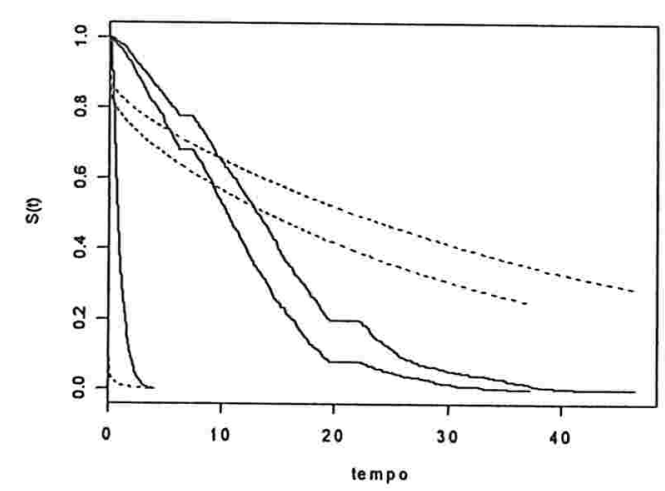

(d)

Figura 6.1: a) risco acumulado do modelo Weibull - b) TTT-"plot"

c) Kaplan-Meier com ajuste Weibull perturbado por uma exponencial

d) Kaplan-Meier com ajuste Weibull

As estimativas de máxima verossimilhança e as estimativas Bayesianas dos parâmetros, assim como seus respectivos intervalos de confiança e credibilidade, considerando o ajuste de um modelo Weibull perturbado por uma exponencial, estão apresentados na Tabela 
(6.1). Os valores estimados para os parâmetros foram baseados em três cadeias simuladas com 18000 iterações cada e um "burn-in" de 4000. Para cada cadeia, foram tomados valores de 3 em 3, obtendo-se assim uma cadeia de tamanho 14000 . Este critério de seleção foi sugerido pelo diagnóstico de convergência de Raftery e Lewis (1992). Através do diagnóstico de convergência de Gelman-Rubin, obtivemos valores de $\hat{R}=1.02 ; 1.02 ; 1.0001,1.03$ para $\beta_{0}, \beta_{1}, \phi$ e $\omega_{0}$, respectivamente. Tais valores trazem fortes indícios de convergência para a densidade a posteriori dos parâmetros. A monitoração da convergência foi feita através do "software" CODA. Utilizando esse número de iterações, os quatro diagnósticos implementados no CODA indicaram a existência de convergência das amostras para cada um dos parâmetros. Podemos dizer que os resultados das análises clássica e Bayesiana foram parecidos.

Tabela 6.1: Estimativas Bayesianas e IC de $95 \%$ para os parâmetros

\begin{tabular}{lcccc}
\hline \hline Procedimento & $\hat{\beta}_{0}$ & $\hat{\beta}_{1}$ & $\omega_{0}$ & $\hat{\phi}$ \\
\hline EMV & 2.81 & 0.92 & 6.33 & 0.44 \\
Int.confiança & $(2.451 ; 3.099)$ & $(0.52 ; 1.249)$ & $(6.25 ; 6.52)$ & $(0.40 ; 0.51)$ \\
MCMC & 2.91 & 0.84 & 6.315 & 0.436 \\
Int.credibilidade & $(2.362 ; 3.267)$ & $(0.49 ; 1.36)$ & $(6.18 ; 6.499)$ & $(0.38 ; 0.499)$ \\
\hline \hline
\end{tabular}

Dados simulados com $\phi_{1}=0.4, \phi_{2}=1, \beta_{0}=2, \beta_{1}=0.8, \rho=-0.65$ e três níveis de voltagem $X_{i}=7,15,20$, com 100 observações em cada nível. 


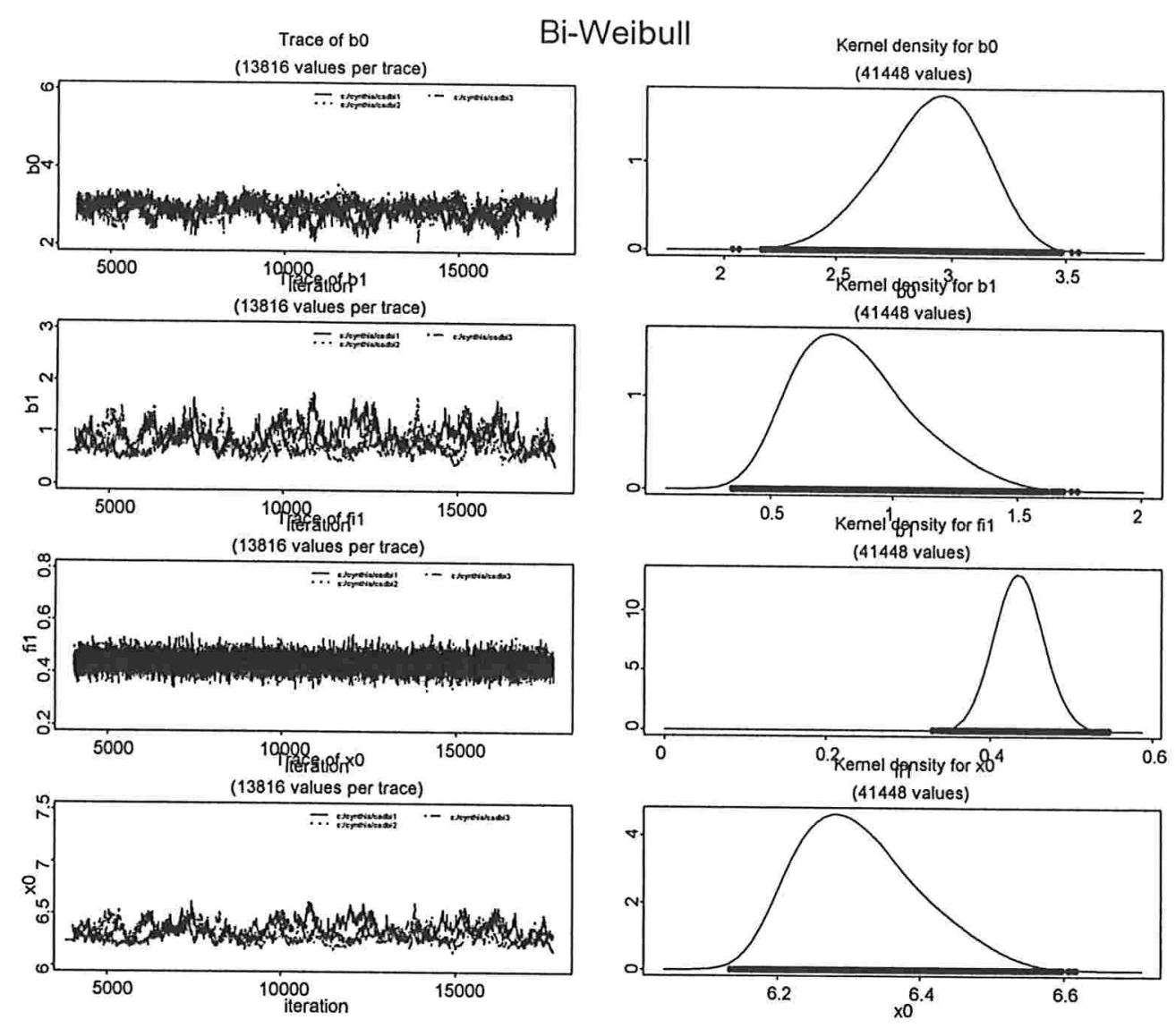

Figura 6.2: Gráficos das posterioris marginais e a história das iterações do algoritmo ARS

\subsection{Conclusões}

Neste capítulo abordamos os modelos de múltiplos riscos, os quais possuem a característica de permitir o ajuste de formas de riscos não monótonas, tais como em forma de "U" e também as multimodais. Também mostramos como identificar a possibilidade de ajuste de tais modelos com base em diagnósticos gráficos, mais precisamente naquele conhecido como TTT-"plot" (Mudholkar et al, 1996; Aarset, 1987), os quais determinam a forma da função de risco.

Apresentamos ainda uma generalização dos modelos de múltiplos riscos, em que o tempo até a falha depende de covariáveis, e utilizamos uma parametrização no modelo de múltiplos riscos proposta por Louzada-Neto (1999).

Especificamente, consideramos os modelos com riscos individuais Weibull e um caso 
particular deste em que o parâmetro de forma no segundo risco Weibull foi escolhido como 1, obtendo assim o que chamamos de um modelo Weibull perturbado por uma exponencial. Nesse mesmo modelo, além de considerar a presença de covariáveis e observações censuradas, incluímos ainda um parâmetro de estresse limiar.

Em um conjunto de dados simulado, fizemos uma análise preliminar através de diagnósticos gráficos e os seguintes resultados foram observados:

1) O gráfico do risco empírico acumulado mostrou uma certa curvatura, indicando que $S(t)$ não poderia ser ajustada por um modelo Weibull.

2) O TTT-plot apresentou regiões convexas e outras aproximadamente côncavas, quase lineares, para todos os níveis de estresse, o que indica a presença de mais de um risco.

3) Através dos gráficos de Kaplan-Meier, observamos que, se for ignorada a presença de mais de um risco, podemos ter problemas nas estimações dos parâmetros caso seja considerado apenas o modelo Weibull simples. Entretanto, observamos um ajuste perfeito para o modelo Weibull perturbado por uma exponencial.

As estimativas dos parâmetros, bem como seus respectivos intervalos de confiança e credibilidade, foram obtidas através de análises clássica e Bayesiana. As estimativas clássicas deram bons resultados, talvez pelo fato de que o número de amostras geradas foi relativamente grande. Os valores das posterioris condicionais foram amostrados via algoritmo ARS, o qual mostrou-se uma ferramenta bastante útil em problemas de estimação Bayesiana. A monitoração da convergência foi feita através do "software" CODA. Utilizando um número de iterações proposto pelo diagnóstico de Raftery e Lewis (1992), os quatro diagnósticos implementados no CODA indicaram a existência de convergência das amostras para cada um dos parâmetros. Podemos dizer que os resultados das análises clássica e Bayesiana foram bastante próximos para todos os parâmetros. 


\section{Apêndices}

\section{A:Métodos de estimação}

A1:O Método de máxima verossimilhança

O método de máxima verossimilhança trata o problema de estimação da seguinte forma: a partir dos resultados obtidos pela amostrá, qual é a distribuição, dentre todas aquelas definidas pelos possíveis valores de seus parâmetros, com maior possibilidade de ter gerado tal amostra? Em outras palavras, se, por exemplo, a distribuição dos tempos de vida é a Weibull $(\phi, \lambda)$, para cada combinação diferente de valores para $\phi$ e $\lambda$ temos diferentes distribuições Weibull. O estimador de máxima verossimilhança escolhe aquele par de valores $\phi$ e $\lambda$ que melhor explique a amostra observada.

Em conceitos matemáticos, suponha, inicialmente, observações $t_{1}, \ldots, t_{n}$ de uma amostra de uma certa população de interesse, em que todas as observações são não-censuradas. Suponha ainda que a população seja caracterizada por sua função de densidade $f(t)$. A função de verossimilhança para um parâmetro genérico, digamos $\theta$, desta população, é dada por

$$
L(\theta)=\prod_{i=1}^{n} f\left(t_{i}, \theta\right)
$$

em que $\theta$ pode estar representando um único ou um conjunto de parâmetros. No modelo Weibull, por exemplo, $\theta=(\phi, \lambda)$. Em termos matemáticos, o problema de determinar "a distribuição que melhor explique a amostra observada" é então o de encontrar um valor de $\theta$ que maximize a função $L(\theta)$.

Quando $t_{1}, \ldots, t_{n}$ representam os tempos de falha ou os tempos de censura observados, as observações podem ser divididas em dois conjuntos: as $r$ primeiras, $(i=1, \ldots, r)$, são as não-censuradas, e as $n-r$ seguintes, $(i=r+1, r+2, \ldots, n)$, as censuradas. Assim, 
a função de verossimilhança pode ser escrita como

$$
L(\theta)=\prod_{i=1}^{r} f\left(t_{i}, \theta\right) \prod_{i=r+1}^{n} S\left(t_{i}, \theta\right)
$$

ou, usando a variável indicadora $\delta_{i}$, em que $\delta_{i}=1$ se $t_{i}$ é exatamente observado ou $\delta_{i}=0$ se $t_{i}$ é censurado, como

$$
\begin{aligned}
L(\theta) & =\prod_{\delta_{i}=1} f\left(t_{i}, \theta\right) \prod_{\delta_{i}=0} S\left(t_{i}, \theta\right) \\
& =\prod_{\delta_{i}=1} h\left(t_{i}, \theta\right) S\left(t_{i}, \theta\right) \prod_{\delta_{i}=0} S\left(t_{i}, \theta\right) \\
& =\prod_{i=1}^{n}\left(h\left(t_{i}, \theta\right)\right)^{\delta_{i}} S\left(t_{i}, \theta\right) .
\end{aligned}
$$

A equação (6.14) (ou (6.15) ) é válida para os mecanismos de censura dos tipos I e II, assim como para o de censura aleatória. Os estimadores de máxima verossimilhança de $\theta$ são os valores $\hat{\theta}$ que maximizam $L(\theta)$ ou, equivalentemente, $\log L(\theta)$, o qual é em geral mais conveniente. Ou seja, eles são encontrados resolvendo-se o sistema de equações

$$
U(\theta)=\frac{\partial \log L(\theta)}{\partial \theta}=0 .
$$

Suponha que, para um certo teste acelerado, $m$ grupos de $n_{i}$ itens, $i=1, \ldots, m$, sejam submetidos a $m$ covariáveis de estresse constantes e fixadas $z_{i}$ (daqui por diante denomimadas niveis de estresse) associadas aos tempos de falha $t_{i j}, j=1, \ldots, n$. Os estimadores de máxima verossimilhança (EMVs) dos parâmetros podem ser obtidos através da maximização direta da função de log-verossimilhança

$$
\begin{aligned}
l(\theta) & =\log L(\theta)=\sum_{i=1}^{m} \sum_{j=1}^{n_{i}} \delta_{i j} \log h\left(t_{i j} \mid z_{i}, \theta\right)+\log S\left(t_{i j} \mid z_{i}, \theta\right) \\
& =\sum_{i=1}^{m} \sum_{j=1}^{n_{i}} \delta_{i j} \log h\left(t_{i j} \mid z_{i}, \theta\right)-H\left(t_{i j} \mid z_{i}, \theta\right),
\end{aligned}
$$

onde $H\left(t_{i j} \mid z_{i}\right)$ é a função de risco base acumulada.

Em todos os exemplos numéricos desta tese, não foi possível encontrar uma forma analítica fechada para os parâmetros dos modelos. Assim, uma alternativa para as estimações dos parâmetros é a maximização direta da função de log-verossimilhança (6.17) via 
métodos de resolução de sistemas não lineares, como, por exemplo, o método de Newton ou alguns de seus variantes: Newton-Raphson, Newton-Raphson Ridge ou quasi-Newton (Hartmann, 1994). Neste trabalho utilizamos o método de quasi-Newton, o qual aparece como sub-rotina em alguns softwares, como, por exemplo, o software $R$.

\section{A2:Inferências sobre os parâmetros}

Inferências sobre os parâmetros $\theta$ do modelo são feitas usualmente através da normalidade assintótica dos estimadores de máxima verossimilhança (Sen e Singer, 1993; Cox e Hinkley, 1974). Assim, para amostras suficientemente grandes e sob certas condições de regularidade, pode-se mostrar que

$$
i^{1 / 2}\left(\theta_{p}\right)\left[\hat{\theta}_{p}-\theta_{p}\right] \stackrel{D}{\longrightarrow} N\left(0, I_{p}\right)
$$

onde $p$ é a dimensão do vetor de parâmetros $\theta, \quad i^{1 / 2}\left(\theta_{p}\right)$ é a matriz raiz quadrada da matriz de informação esperada de Fischer e $I_{p}$ é a matriz identidade de dimensão $p$. A distribuição assintótica dos EMV's continua válida se a matriz de informação esperada de Fischer for substituída pela matriz de informação observada, a qual é dada por

$$
I(\hat{\theta})=\left[\begin{array}{cccc}
-\frac{\partial^{2} l}{\partial \theta_{1}^{2}} & -\frac{\partial^{2} l}{\partial \theta_{1} \partial \theta_{2}} & \ldots & -\frac{\partial^{2} l}{\partial \theta_{1} \theta_{p}} \\
& -\frac{\partial^{2} l}{\partial \theta_{2}^{2}} & \ldots & -\frac{\partial^{2} l}{\partial \theta_{2} \theta_{p}} \\
& & \vdots & \vdots \\
\text { simétrica } & & & -\frac{\partial^{2} l}{\partial \theta_{p}^{2}}
\end{array}\right] \mid \theta=\hat{\theta}
$$

Considerando a normalidade assintótica dos estimadores de máxima verossimilhança $\hat{\theta}$, pode-se calcular os intervalos de confiança para o vetor de parâmetros $\theta$ e para a função de sobrevivência $S\left(t_{0}\right)$ para dado $t_{0}$. Representando a inversa da matriz de informação observada por

$$
I^{-1}(\hat{\theta})=\left[\begin{array}{cccc}
b_{11} & b_{12} & \ldots & b_{1 p} \\
b_{21} & b_{22} & \ldots & b_{2 p} \\
\vdots & \vdots & \vdots & \vdots \\
b_{p 1} & b_{p 2} & \ldots & b_{p p}
\end{array}\right]
$$

e considerando um nível de confiança de $100(1-\alpha) \%$, os intervalos de confiança aproximados para $\theta$ são dados por 


$$
\begin{gathered}
I C\left(\theta_{1}\right)=\left[\hat{\theta}_{1}-z_{\alpha / 2}\left(b_{11}\right)^{1 / 2} ; \hat{\theta}_{1}+z_{\alpha / 2}\left(b_{11}\right)^{1 / 2}\right] \\
I C\left(\theta_{2}\right)=\left[\hat{\theta}_{2}-z_{\alpha / 2}\left(b_{22}\right)^{1 / 2} ; \hat{\theta}_{2}+z_{\alpha / 2}\left(b_{22}\right)^{1 / 2}\right] \\
\vdots \\
I C\left(\theta_{p}\right)=\left[\hat{\theta}_{p}-z_{\alpha / 2}\left(b_{p p}\right)^{1 / 2} ; \hat{\theta}_{p}+z_{\alpha / 2}\left(b_{p p}\right)^{1 / 2}\right],
\end{gathered}
$$

onde $z_{\alpha / 2}$ é o percentil correspondente da distribuição normal padronizada e $b_{i i}$ é o $i$ ésimo elemento da diagonal principal da inversa da matriz de informação observada $I$ calculada nos EMV's.

\section{A3:Análise Bayesiana para modelos de tempos de vida}

$\mathrm{Na}$ análise de confiabilidade ou de sobrevivência, quando adotamos um modelo para os dados de tempos de vida, temos interesse em determinar o valor de determinado parâmetro que caracteriza tal modelo. Embora esse parâmetro seja considerado fixo, não temos certeza do seu verdadeiro valor e, assim, procuramos uma forma de, através dos dados, expressar essa incerteza.

Uma alternativa é considerar uma distribuição para o parâmetro de interesse que, sob esse ponto de vista, é considerado uma variável aleatória (veja, por exemplo, Box e Tiao, 1973). Com essa formulação, usamos o teorema de Bayes, o qual fornece um método para "calcular" a distribuição do parâmetro, expressando nossa incerteza condicionada aos dados.

\section{A3.1:Teorema de Bayes}

Suponha que $t=\left(t_{1}, \ldots, t_{n}\right)$ seja um vetor de $n$ observações e suponha que a distribuição de probabilidade $\pi(t \mid \theta)$ dependa de $p$ parâmetros. A quantidade $\theta=\left(\theta_{1}, \ldots, \theta_{p}\right)$, sob o ponto de vista Bayesiano, é considerada uma variável aleatória, representando características que se deseja conhecer. Se $\theta$ tem uma distribuição de probabilidade (denominada distribuição a priori) $\pi(\theta)$, então,

$$
\pi(t \mid \theta) \pi(\theta)=\pi(t, \theta)=\pi(\theta \mid t) \pi(t)
$$

Assim, a distribuição de $\theta$, dadas as observações, pode ser expressa como

$$
\pi(\theta \mid t)=\frac{\pi(t \mid \theta) \pi(\theta)}{\pi(t)}
$$


onde $\pi(t \mid \theta)$ denota a função de verossimilhança $L(\theta)$ e

$$
\pi(t)=c^{-1}=\int_{\Theta} \pi(t \mid \theta) \pi(\theta) d \theta .
$$

Dizemos que $c$ é a constante normalizadora de $\pi(\theta \mid t)$. Pelo teorema de Bayes, podemos escrever a densidade a posteriori $\pi(\theta \mid t)(6.23)$ como

$$
\pi(\theta \mid t)=c L(\theta) \pi(\theta)
$$

ou

$$
\pi(\theta \mid t) \propto L(\theta) \pi(\theta)
$$

O procedimento de inferência Bayesiana baseia-se na distribuição a posteriori das componentes de $\theta$. Neste ponto surgem duas dificuldades, a primeira na obtenção da distribuição a posteriori marginal e a segunda no cálculo de momentos a posteriori de interesse. Em ambos os casos, é necessária a resolução de integrais que, muitas vezes, não admitem uma solução analítica. Nesses casos, métodos numéricos (veja, por exemplo, Naylor e Smith, 1982) ou métodos de aproximação de integrais, tais como os métodos de Laplace (Tierney e Kadane, 1986), são necessários. Ocorre que, para a aplicação deste último, é necessário determinar o valor que maximiza a função de densidade a posteriori e, assim, à medida que a dimensão do espaço paramétrico aumenta, aumentam também as dificuldades para sua obtenção, e a aplicação do método torna-se cada vez mais difícil de ser implementada. Neste sentido, uma alternativa é utilizar os algoritmos de simulação estocástica de Monte Carlo via Cadeias de Markov (MCMC), tais como os algoritmos de Gibbs (Gelfand e Smith, 1990) e Metropolis-Hastings (Chib e Grenberg, 1995). Esses métodos permitem, até mesmo, escolher densidades a priori sem perder a propriedade de conjugação.

O algoritmo do amostrador de Gibbs (A.G.) e o algoritmo de Metropolis-Hastings são métodos de simulação de Monte Carlo via Cadeias de Markov (MCMC). O amostrador de Gibbs é uma técnica de MCMC para gerar amostras de uma densidade multivariada não normalizada. Gelfand e Smith (1990) popularizaram o A.G. na aplicação do método na área de inferência Bayesiana. A seguir, descrevemos brevemente o procedimento do amostrador de Gibbs.

\section{A3.3:Amostrador de Gibbs}


Suponha que desejemos estimar $\pi\left(\theta_{1}, \theta_{2}, \ldots, \theta_{p} \mid\right.$ dados $)$, a densidade conjunta a posteriori de $\left(\theta_{1}, \theta_{2}, \ldots, \theta_{p}\right)$, a partir dos dados. O algoritmo considera que as densidades condicionais a posteriori de cada parâmetro $\theta_{i}$, dados todos os outros, sejam conhecidas e disponíveis. Essas distribuições são denotadas por $\pi\left(\theta_{1} \mid \theta_{2}, \ldots, \theta_{p} ;\right.$ dados $), \pi\left(\theta_{2} \mid \theta_{1}, \theta_{3}, \ldots, \theta_{p} ;\right.$ dados $), \ldots$, $\pi\left(\theta_{p} \mid \theta_{1}, \ldots, \theta_{p-1} ;\right.$ dados $)$. O algoritmo segue os seguintes passos: $i=1$;

Passo 0: Arbitre um valor inicial $\theta^{(0)}=\left(\theta_{1}^{(0)}, \ldots, \theta_{p}^{(0)}\right)$ e inicialize o contador de iteração

Passo 1: Obtenha um novo valor $\theta^{(i)}=\left(\theta_{1}^{(i)}, \ldots, \theta_{p}^{(i)}\right)$ a partir de $\theta^{(i-1)}$ através de sucessivas gerações de valores:

$$
\begin{aligned}
& \operatorname{gerar} \theta_{1}^{(i)} \sim \pi\left(\theta_{1} \mid \theta_{2}^{(i-1)}, \ldots, \theta_{p}^{(i-1)} ; \text { dados }\right) \\
& \text { gerar } \theta_{2}^{(i)} \sim \pi\left(\theta_{2} \mid \theta_{1}^{(i)}, \theta_{3}^{(i-1)}, \ldots, \theta_{p}^{(i-1)} ; \text { dados }\right) \\
& \vdots \\
& \operatorname{gerar} \theta_{p}^{(i)} \sim \pi\left(\theta_{p} \mid \theta_{1}^{(i)}, \ldots, \theta_{p-1}^{(i)} ; \text { dados }\right)
\end{aligned}
$$

Passo 2: Incremente $i$ e volte para o passo 1 até atingir a convergência.

A medida que o número de iterações aumenta, a cadeia se aproxima de sua condição de equilíbrio. Admite-se então que a convergência é atingida em uma iteração cuja distribuição esteja arbitrariamente próxima da distribuição de equilíbrio. Sob certas condições de regularidade (veja, por exemplo, Tierney, 1994), essa cadeia de Markov converge para uma distribuição estacionária para $k$ suficientemente grande, isto é, $\left(\theta_{1}^{(k)}, \ldots, \theta_{p}^{(k)}\right)$ tem uma distribuição aproximadamente igual a $\pi\left(\theta_{1}, \theta_{2}, \ldots, \theta_{p} \mid\right.$ dados $)$. Além disso, $\theta_{i}$ pode ser considerada como uma observação simulada de $\pi\left(\theta_{i} \mid d a d o s\right)$, a distribuição marginal de $\theta_{i}$. Replicações paralelas independentes de todo o algoritmo acima $N$ vezes produz em $N$ conjuntos de vetores dos parâmetros e, então, para cada elemento de $\theta$ obtemos uma amostra simulada de tamanho $N$ da distribuição a posteriori conjunta.

\section{A3.4:Metropolis-Hastings}

Quando as distribuições condicionais a posteriori não são facilmente identificadas como possuidoras de uma forma padrão (normal, gama, etc.), o que impossibilita a geração direta a partir destas distribuições, usa-se o algoritmo Metropolis (Metropolis et al., 1953). Uma generalização do algoritmo dado por Hastings (1970) é descrita aqui. Esta técnica requer uma densidade de transição $q\left(\theta, \theta^{*}\right)$ que, não necessariamente, tenha probabilidade de equilíbrio $\pi$, mas que represente uma regra de passagem que define uma cadeia. Considere, também, a probabilidade de aceitação $p\left(\theta^{(r-1)}, \theta^{*}\right)$ definida abaixo. O algoritmo segue os seguintes passos: 
Passo 0: arbitre um valor inicial $\theta=\theta^{(0)}$; inicialize o contador de iteração $r=1$;

Passo 1: mova a cadeia para um novo valor de $\theta^{*}$ gerado da densidade $q\left(\theta^{(r-1)}, \theta^{*}\right)$;

Passo 2: gere $u$ da uniforme $(0,1)$;

Passo 3: aceite o valor $\theta^{*}$ gerado se

$$
u \leq p\left(\theta^{(r-1)}, \theta^{*}\right)=\min \left(1, \frac{\pi\left(\theta^{*}\right) q\left(\theta^{*}, \theta^{(r-1)}\right)}{\pi\left(\theta^{r-1}\right) q\left(\theta^{(r-1)}, \theta^{*}\right)}\right) .
$$

Caso contrário, fique $\operatorname{com} \theta^{(r)}=\theta^{(r-1)}$;

Passo 4: atualize o contador $r$ para $r+1$, e volte para o passo 1 até que a distribuição estacionária seja atingida.

Para $r$ suficientemente grande, $\theta^{(r)}$ é uma amostra da distribuição a posteriori $\pi(\boldsymbol{\theta})$.

Para o caso vetorial $\boldsymbol{\theta}=\left(\theta_{1}, \theta_{2}, \ldots, \theta_{p}\right)$, teremos a densidade de transição dada por $q\left(\theta, \theta^{*}\right)$ e uma probabilidade de aceitação dada por $p\left(\theta^{(r-1)}, \theta^{*}\right)$. Depois, deve-se proceder como na forma acima.

Observe que, para este algoritmo, temos que conhecer a forma funcional da função de verossimilhança e a densidade a priori, mas não a constante normalizadora.

\section{A3.5:O Método de Rejeição Adaptativa}

Amostragem por rejeição adaptativa, usada comumente na amostragem Gibbs (Gilks \& Wild, 1992), é um método para amostrar, de forma eficiente, densidades univariadas complicadas, como geralmente ocorre em aplicações do amostrador de Gibbs.

Como descrição geral, o método $A R S$ constrói uma função envelope da densidade proposta, a qual é usada na amostragem por rejeição. Quando um ponto é rejeitado pelo ARS, o envelope é atualizado para corresponder de forma mais próxima à verdadeira densidade, reduzindo portanto a chance de rejeição dos pontos subsequentes. Em geral, trabalha-se com o log da função densidade, pela facilidade computacional. Em termos gerais, uma distribuição a posteriori $\pi(\theta \mid d a d o s)$ é simulada adaptando ou uma função envelope ou uma função "squeezing " para o núcleo dessa distribuição a posteriori avaliado num conjunto de abcissas $\mathcal{A}$. Seja $K(\theta)$ o núcleo da distribuição a posteriori em questão, para o qual se requer uma função "envelope " $b(\theta) \geq K(\theta), \forall \theta \in \Theta$, e uma função "squeezing " $a(\theta)$, tal que $a(\theta) \geq K(\theta), \forall \theta \in \Theta$. Assim, a aceitação de valores $\theta^{\prime}$ na amostragem de $\theta$ deve respeitar os seguintes passos:

i) escolha de um conjunto de abscissas $\mathcal{A}$;

ii) geração de um valor $\theta^{\prime}$ a partir $\operatorname{de} b(\theta)$ em $\mathcal{A}$; 
ii) simulação de uma variável aleatória $u$ com distribuição uniforme $(0,1)$;

iv) se $U \leq a\left(\theta^{\prime}\right) / b\left(\theta^{\prime}\right)$ em $\mathcal{A}$, segue-se para o passo seguinte; caso contrário, retorna-se a ii) e inclui-se $\theta^{\prime}$ em $\mathcal{A}$;

v) Se $u \leq a\left(\theta^{\prime}\right) / b\left(\theta^{\prime}\right)$ em $\mathcal{A}$, aceita-se $\theta^{\prime}$ e inclui-se $\theta^{\prime}$ em $\mathcal{A}$.

$\mathrm{Na}$ versão original do ARS, a função envelope é construída a partir de um conjunto de retas tangentes da função log densidade (Gilks \& Wild, 1992). Em uma versão mais recente, o envelope é construído a partir de retas secantes, interseccionando no log da densidade. Quanto menos rejeição acontece, menos pontos são avaliados no log da densidade. As avaliações são computacionalmente caras nas aplicações do amostrador de Gibbs.

O algoritmo ARS está implementado no software BUGS (Spiegelhalter et al., 1997). Para a sua utilização, devemos então mostrar que as densidades são log-côncavas, o que é feito calculando-se as derivadas de segunda ordem das posteriores condicionais com respeito aos parâmetros $\left(\theta_{1}, \theta_{2}, \ldots, \theta_{p}\right)$ e mostrando que todas elas são negativas, ou seja, devemos mostrar que

$$
\begin{aligned}
\frac{\partial^{2} \pi\left(\theta_{1} \mid \theta_{2}, \ldots, \theta_{p} ; \text { dados }\right)}{\partial \theta_{1}^{2}}<0 \\
\frac{\partial^{2} \pi\left(\theta_{2} \mid \theta_{1}, \theta_{3}, \ldots, \theta_{p} ; \text { dados }\right)}{\partial \theta_{2}^{2}}<0 \\
\vdots \\
\frac{\partial^{2} \pi\left(\theta_{p} \mid \theta_{1}, \ldots, \theta_{p-1} ; \text { dados }\right)}{\partial \theta_{p}^{2}}<0
\end{aligned}
$$

Para o caso de distribuições que não sejam log-côncavas, Gilks et al., (1995) apresentaram um método de amostragem por rejeição adaptativa -ARMS, utilizando um passo com o algoritmo Metropolis-Hastings (Metropolis et al., 1953) em cada ponto aceito pelo ARS. No passo Metropolis, o novo ponto é comparado cọm o ponto anterior amostrado. Se o novo ponto é rejeitado, então o ponto anterior é considerado como o novo ponto.

O algoritmo ARMS é uma generalização do método de rejeição adaptativo ARS (Gilks e Wild, 1992), pois inclui um esquema de amostragem semelhante ao usado no algoritmo Metropolis-Hastings e, por meio deste, é possível amostrar a partir de densidades não log-côncavas. Para densidades log-côncavas, a taxa de aceitação do algoritmo ARMS é de $100 \%$ (Gilks et al, 1995). Assim, avaliar a log-concavidade utilizando-se do algoritmo ARMS torna-se desnecessário. 


\section{B :Diagnósticos de Convergência}

Quando utilizamos métodos de MCMC, podemos ter algumas dificuldades em fazer inferências, tais como o desconhecimento do número de iterações necessário para se obter convergência, o fato de que as iterações iniciais da amostra podem ser influenciadas pelos valores iniciais dos parâmetros e ainda o fato de que a sequência de valores pode apresentar uma forte correlação entre os parâmetros. Não existe uma técnica geral para resolver essas questões, porém existem métodos de verificação de convergência, baseados nas propriedades da cadeia de Markov, para indicar a convergência, ou não, da amostra simulada para a distribuição marginal.

Os diagnósticos de convergência usados neste trabalho são: Geweke (1992), GelmanRubin (Gelman e Rubin,1992), Raftery e Lewis (1992) e Heidelberger e Welch (1983), os quais estão resumidamente apresentados no Apêndice B. Eles estão implementados na biblioteca CODA (Convergence Diagnosis and Output Analysis Software for Gibbs Sampling Output) (Best et al., 1996). Em geral, esses métodos são utilizados conjuntamente a fim de se obter uma indicação de convergência, uma vez que nenhum deles pode ser considerado o melhor dentre todos.

Nesta tese, os critérios de convergência foram utilizados da forma descrita a seguir. Analisamos as cadeias geradas por meio do software Winbugs através da biblioteca CODA e utilizamos primeiramente o critério de Gelman-Rubin. Nos casos em que o valor encontrado para o fator de redução $\hat{R}=\sqrt{\hat{V}(t(\theta)) / W}$ foi menor do que 1.2 , onde $\hat{V}$ é a variância de $T$ e $W$ é a variância dentro das cadeias (veja critérios de convergência no apêndice), aceitamos a convergência e, caso contrário, acessamos o critério de Raftery e Lewis, o qual fornece o número de iterações iniciais que devem ser descartadas como "burn-in", o tamanho da cadeia a ser gerada e o espaçamento a ser considerado entre as iterações. De acordo com o espaçamento sugerido, o qual chamamos de "lag", obtivemos uma nova amostra de tamanho menor, a partir da qual fizemos novamente toda a análise. Geralmente, utilizando o "lag" sugerido, o critério de Raftery e Lewis apresentou convergência para essa nova amostra, assim como os outros critérios dados por Gelman e Rubin e por Geweke.

Aqui é apresentado um resumo dos diagnósticos utilizados neste trabalho para avaliar a convergência das cadeias geradas utilizando métodos MCMC. Os três critérios estão implementados na biblioteca CODA. Para maiores detalhes, ver, por exemplo, Brooks e Roberts (1995), Gamerman (1996) e Best et al. (1996). 


\section{B.1. Diagnóstico de Gelman e Rubin}

Este método proposto por Gelman e Rubin (1992) utiliza cadeias múltiplas, inicializadas em pontos distintos, comparando a variância amostral dentro e entre as cadeias, para cada uma das quantidades de interesse. Esta comparação é usada para estimar o fator pelo qual o parâmetro de escala da distribuição a posteriori marginal pode ser reduzido, quando o número de iterações é grande. Para a obtenção de um resultado melhor no teste, as quantidades de interesse devem ter distribuição aproximadamente normal.

A inspeção visual de similaridade entre as trajetórias das várias cadeias após um certo número de iterações certamente é um indicio forte de convergência. Gelman \& Rubin (1992) formalizaram essa idéia, de que as trajetórias das cadeias devem ser a mesma depois de convergirem, através do uso de técnicas de análise de variância. A idéia geral é testar se a dispersão intra-cadeias é maior do que a a dispersão inter-cadeias. Isto equivale a dizer que: o histograma das cadeias como um todo deve ser similar aos histogramas das cadeias tomadas individualmente.

Considerando $m$ cadeias que evoluem em paralelo e uma função real $t(\theta)$, tem-se $m$ trajetórias $\left\{t_{i}^{(1)}, t_{i}^{(2)}, \ldots, t_{i}^{(n)}\right\}, i=1, \ldots, m$ para $t$. Portanto, podem ser obtidas a variância entre as cadeias, $B$, e a variância dentro das cadeias, $W$. As fórmulas correspondentes são dadas por:

$$
B=\frac{n}{m-1} \sum_{i=1}^{m}\left(\bar{t}_{i}-\bar{t}\right) \quad \text { e } \quad W=\frac{1}{m(m-1)} \sum_{i=1}^{m} \sum_{j=1}^{n}\left(t_{i}^{(j)}-\bar{t}_{i}\right)^{2}
$$

onde $\bar{t}_{i}$ é a media das observações da cadeia $i$ e $\hat{t}$ é a média dessas médias, $1=1, \ldots, m$.

Sob condição de convergência, todos os $m . n$ valores serão gerados da posteriori e a variância de $t$ pode ser estimada de forma não viciada por:

$$
V(t(\theta))=\left(1-\frac{1}{n}\right) W+\left(\frac{1}{n}\right) B
$$

Se as cadeias ainda não estiverem convergido então essa estimativa é maior que $V(t(\theta))$, pois os valores iniciais ainda estão sendo influenciados pelos valores dos ou-tros parâmetros da cadeia, de forma que a distribuição de equilíbrio ainda não foi atingida, indicando que eles foram escolhidos com dispersão maior que da distribuição de equilíbrio. Por outro lado, $W$ fornece estimativas menores que $V(t(\theta))$, pois a cadeia não terá coberto toda a variabilidade de $t(\theta)$.

Um indicador de convergência é dado pela chamada redução potencial estimada da 
escala $\hat{R}=\sqrt{\hat{V}(t(\theta)) / W}$, que é sempre maior que 1 . A medida que $n$ cresce, ambos os estimadores acabarão convergido para $V(t(\theta))$, e $R$ convergirá para 1. Assim, $\hat{R}$ pode ser usado como indicador de convergência pela avaliação de sua proximidade a 1. Gelman (1995) sugere aceitar como garantia de convergência valores de $\hat{R} \leq 1.1$.

\section{B.2. Diagnóstico de Geweke}

O diagnóstico proposto por Geweke (1992) é baseado em métodos de séries temporais. Ele foi desenvolvido para indicar a convergência da média a posteriori de uma função da quantidade amostrada, $g(\theta)$, em uma única cadeia, isto é, os valores $g\left(\theta^{(j)}\right)$ são calculados a cada iteração da simulação, formando uma série temporal. Estima-se, a partir da cadeia, a variância assintótica $S_{g}(0)$ da medida de $g(\theta)$.

Resumidamente, o diagnóstico é dado da seguinte forma:

Divida a cadeia gerada de tamanho $n$ em duas sequências, onde a primeira possui as $n_{a}$ primeiras iterações e a segunda, as $n_{b}$ últimas iterações, e calculem as médias $m_{a}$ e $m_{b}$ e variâncias assintóticos $\hat{S}_{a}(0)$ e $\hat{S}_{b}(0)$, respectivamente, sendo que a variância é determinada pela estimação da densidade espectral. Se a cadeia completa for estacionária, as médias dessas sequências estarão próximas. Então, para as razões $\frac{n_{a}}{n}$ e $\frac{n_{b}}{n}$ fixas, temos $\frac{\left(n_{a}+n_{b}\right)}{n}<1$.

Assim, quando $n \rightarrow \infty$,

$$
\frac{m_{a}-m_{b}}{\sqrt{\frac{\hat{S}_{a}(0)}{n_{a}}+\frac{\hat{S}_{b}(0)}{n_{b}}}} \sim N(0,1),
$$

se a sequência gerada for estacionária. Geweke segere que a aplicação deste teste seja feita descartando-se algumas iterações iniciais e, ainda, que $n_{a}=0.1 n$ e $n_{b}=0.5 n$.

Resultados melhores podem ser obtidos quando a distribuição a posteriori marginal dos parâmetros for aproximadamente normal. Este teste pode ser generalizado para cadeias múltiplas.

\section{B.3. Diagnóstico de Raftery e Lewis}

Este método proposto por Raftery \& Lewis (1992) se aplica a uma única cadeia. Ele tem por objetivo detectar convergência e fornecer limites para a precisão dos quantis estimados para as funções das quantidades de interesse. Fornece também o número de iterações iniciais a serem descartadas como burn-in, o tamanho da cadeia a ser gerada considerando uma determinada precisão na estimativa; o espaçamento entre as iterações a serem consideradas; e uma medida, denotada por $I$, que é dada pela razão entre o 
número total de iterações e o número mínimo exigido para a precisão desejada. Para valores de $I$ maior do que 1 , existe indicação de alta correlação dentro da amostra gerada e provavelmente uma falha na convergência da cadeia, onde, por exemplo, reparametrizações ou diferentes valores iniciais podem resolver o problema da convergência. 


\section{Referências Bibliográficas}

[1] Aarset, M. V. (1987). How to Identify a Bathtub Hazard Rate. IEEE Transactions on Reliability, v.36, 106-108.

[2] Basu, A. P. and Klein, J. P. (1982). Some Recent Development in Competing Risks Theory. Survival Analysis: Crowley, J. and Johnson, R. A., Hayward: IMS. 216-226.

[3] Barlow, R.E. and Proschan, F. (1975). Statistical Theory of Reliability and Life Testing. New York: Holt, Rinehart and Winston.

[4] Berger, J. M. and Sun, D. O. (1993). Bayesian Analysis for the Poly-Weibull Distribution. Journal of the American Statistical Association, v.88,424,1412-1418.

[5] Berger, J. M. and Sun, D. O. (1996). Bayesian Inference for a Class of Poly-Weibull Distributions. Bayesian Analysis in Statistics and Econometrics, Essays in Honor of Arnold Zellner. New York: Willey: Berry, D. A., Chaloner, K.M. and Geweke, J. K., 101-113.

[6] Best, N. G. Cowles, M. K., and Vines, S.K., (1996).CODA: Convergence Diagnostic and Output Analysis Software for Gibbs Sampling Output, Version 0.3. MRC Biostatistics Unit, Cambridge.

[7] Ciampi, A. and Etezadi-Amoli, J. (1985). A General Model for Testing the Proportional Hazards and the Accelerated Failure Time Hypotheses in the Analysis of Censored Survival Data with Covariates. Communications in Statistics: Theory and Method, v. 14 (3), 651-667.

[8] Collett, D. (1994). Modelling Survival Data in Medical Research. New York: Chapman and hall.

[9] Cox, D. R. (1972). Regression models and life-tables (with discussion). Journal of Royal Statistical Society, B, 34, 187-220. 
[10] Cox, D. R. and Hinkley, D. V. (1974). Theoretical Statistics. London: Chapman and Hall.

[11] Cox, D. R. and Oakes, D. (1984). Analysis of Survival Data. London: Chapman and Hall.

[12] Cox, C. (1987). Threshold Dose-Response Models in Toxicology. Biometrics 43, 511523.

[13] David, H. A. and Moeschberger, M. (1978). The Theory of Competing Risks. New York: MacMil.

[14] Davison, A.C., Hinkley, D.V. (1997). Bootstrap Methods and their Application. Cambridge: Cambridge University Press, 582p

[15] Davison, A.C. and Louzada-Neto, F. (2000). Inference for the Poly-Weibull Model. The Statistician, v. 49, 189-196.

[16] DePriest, D. J. and Launer, R.L. (1983). Reliability in the Acquisitions Process. Lecture Notes in Statistics, vol. 4, New York: Marcel Dekker.

[17] Efron, B. (1979). Bootstrap metthods: another look at the jackknife. Annals of Statistics, 7, 1-26.

[18] Efron, B. and Tibshirani, R. J. (1983). An Introduction to the Bootstrap. New York: Wiley, 436p.

[19] Elandt-Johnson, R. C. and Johnson, N. L. (1980). Survival Models and Data Analysis. New York: Wiley.

[20] Etezadi-Amoli, J. and Ciampi, A. (1987). Extended hazard regression for censored survival data with covariates: a spline approximation for the baseline hazard function. Biometrics, 43, 181-192.

[21] Farewell, V.T. and Prentice, R.L. (1977). A Study of Distributional Shape in Life Testing. Technometrics, 19, 69-75.

[22] Feigl, P. and Zelen, M. (1965). Estimation of Exponential Survival Probabilities with Concomitant Information. Biometrics, v. 21, 826-838. 
[23] Gelfand, A. E. G. and Smith, A. F. M. (1990). Sampling-based approaches to calculating marginal densities. Journal of the American Statistical Association 85, 398-409.

[24] Gelfand, A. E. et al. (2000). Proportional Hazards Models: a Latent Competing Risk Approach. Journal of the Royal Statistical Society, C, v.49, 3, 385-397.

[25] Gelman, A. and Rubin, D. B. (1992). Inference from Iterative Simulation Using Multiple Sequences. Statistical Science, v. 7, 457-511.

[26] Geweke, J. (1992). Evaluating the Accuracy of Sampling-Based Approaches to Calculating Posterior Moments. Bayesian statistics 4: Oxford: Clarendon Press.

[27] Gilks, W. R. and Wild, P. (1992). Adaptive rejection sampling for Gibbs sampling. Applied Statistics 41, 337-348.

[28] Gilks, W., Best, N. G. and Tan, K. K. C. (1995). Adaptive rejection Metropolis sampling within Gibbs sampling. Applied Statistics, 44, 455-472.

[29] Gilks, W.R., Richardson, S. and Spiegelhalter, D.J. (Eds) (1996). Markov Chain Monte Carlo in Practice. London: Chapman and Hall.

[30] Gross, A. J. and Clark, V.A. (1975). Survival Distributions: Reliability Applications in the Biomedical Sciences. New York: Wiley.

[31] Harger, H.W. and Bain, L.J. (1970). Inferential Procedures for the Generalized Gamma Distributions. American Statistical Association, 65, 1601-1609.

[32] Hartmann, W. M. (1994). The NLP Procedure: Release 6.10 and 6.11, extended user's Guide. SAS Campus Drive: Cary, NC 27513, USA: SAS Institute, Inc.

[33] Hastings. W. K. (1970). Monte Carlo sampling methods using Markov chains and their aplications. Biometrika, 57.97-109.

[34] Heidelberger, P. and Welch, P. (1983). Simulation Run Length Control in the Presence of an Initial Transient. Operations Research, v. 31, 1109-1144.

[35] Hirose, H. (1993). Estimation of Threshold Stress in Accelerated Life-Testing. IEE Transactions on Reliability, 42, 650-657.

[36] Kalbfleisch, J. D. and Prentice, R. L. (1980). The Statistical Analysis of Failure Time Data. New York: John Wiley and Sons. 
[37] Kaplan, E. L. and Meier, P. (1958). Nonparametric Estimation from Incomplete Observations. J. Am. Statist. Association, 53, 457-481.

[38] Klein, J. P. and Moeschberger, M. L. (1997). Survival Analysis: Techniques for Censored and Truncated Data. New York: Springer-Verlag.

[39] Lawless, J.F. (1980). Inference in the Generalized Gamma and Log-gamma Distributions. Technometrics, 22, 409-419.

[40] Lawless, J.F. (1982). Statistical Models and Methods for Life Time Data. New York: Jonh Wiley.

[41] Louzada-Neto. F. (1997). Extended hazard regression model for reability and survival analysis. Lifetime Data Analysis, 3, 367-381.

[42] Louzada-Neto. F. (1999). Polyhazard Regression Models for Lifetime Data. Biometrics, v.55, 1281-1285.

[43] Mann, N.R., Schafer, R.E. and Singpurwalla, N. D. (1974). Methods for Statistical Analysis of Reability and Life Test Data. Jonh Wiley \& Sons, New York.

[44] Meeker, W. Q. and Escobar, L. A. (1998). Statistical Methods for Reability Data. Wiley \& Sons, New York.

[45] Metropolis, N. et al. (1953). Equation of State Calculations by Fast Computing Machines. Journal of Chemical and Physics, v. 21, 1087-1091.

[46] Miké, V. and Stanley, K.E. (1982). Statistics in Medical Research. New York: Wiley.

[47] Miller, R. G. (1981). Survival Analysis. Wiley: New York.

[48] Mudholkar, G. S., Srivastava, D. K. and Kolia, G. D. (1996). A Generalization of the Weibull Distribution with Application to the Analysis of Survival Data. Journal of the American Statistical Association, v. 91, 436, 1575-1583.

[49] Peto, R., Pike, M.C., Armitage, P., Breslow, N.E., Cox, D.R., Howard, S.V., Mantel, N., McPherson, K., Peto, J. and Smith, P.G. (1976). Design and Analysis of Randomized Clinical Trials Requiring Prolonged Observation of each Patient. I. Introduction and Design. Br. J. Cancer, 34, 585-612. 
[50] Peto, R., Pike, M.C., Armitage, P., Breslow, N.E., Cox, D.R., Howard, S.V., Mantel, N., McPherson, K., Peto, J. and Smith, P.G. (1977). Design and Analysis of Randomized Clinical Trials Requiring Prolonged Observation of each Patient. II.Analysis and Examples. Br. J. Cancer, 35, 1-39.

[51] Raftery, A. L. and Lewis, S. (1992). How Many Iterations in the Gibbs Sampler? Bayesian Statistics 4, Oxford: Clarendon Press, 763-774.

[52] Naylor, J.C. and Smith, A.F.M. (1990). Applications of a Method fo Efficient Computation od Posterior Distributions. Applied Statistics, 31, 214-225.

[53] Nelson, W. (1982). Applied Life Data Analysis. New York: Wiley.

[54] Nelson, W. (1990). Accelerated Testing- Statistical Models, Test Plans and Data Analysis, John Wiley \& Sons.

[55] Prentice, R.L. (1974). A Log-gamma Model and its Maximum Likelihood Estimation. Biometrica, 61, 539-544.

[56] Prentice, R. L., (1978). Linear rank tests with right censored data. Biometrika, 65, $167-179$.

[57] Sen, P.K. and Singer, J.M. (1993). Large Sample Methods in Statistics: An introduction with Applications. Chapman \& Hall, New York.

[58] Serfling, R. J. (1980). Approximation Theorems of Mathematical Statistics. New York: John Wiley \& Sons.

[59] Smith, R.L. (1991). Weibull Regression Models for Reliability Data. Reliability Engineering and System Safety, 34, 55-77..

[60] Spiegelhalter, D., Thomas, A., Best, N. and Gilks, W. (1997). BUGS- Bayesian inference using Gibbs sampling: Version 0.6. Cambrigde: MRC Bioestatistics Unit.

[61] Stacy, E. W. (1962). A Generalization of the Gamma Distribution. The Anall of Mathematical Statistics, v. 33, 1187-1192.

[62] Tierney, L. , Kass. R. E. and Kadane, J. B. (1989). Aproximate marginal densities for nonlinear functions, Biometrika, 76, 425-433. 
[63] Tierney, L. (1994). Markov Chains of Exploring Posterior Distributions. Annals of Statistics, v. 22, 1701-1762.

[64] Tojeiro, C.A.V., Louzada-Neto, F. and Bolfarine, H. (2004). A Bayesian Analysis for Accelerated Lifetime Tests Under an Exponential Power Law Model with Threshold Stress. Applied Statistics, 31, 685-691.

[65] Weibull, W. (1951). A statistitical distribution function of wide applicability. J. Appl. Mech., 18, 293-297. 